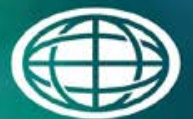

Savannah River

National Laboratory ${ }^{m}$

OPERATED BY SAVANNAH RIVER NUCLEAR SOLUTIONS

\title{
Interpretation of Vadose Zone Monitoring System Data near Engineered Trench 1
}

G. P. Flach

T. S. Whiteside

December 12, 2016

SRNL-STI-2016-00546 


\section{DISCLAIMER}

This work was prepared under an agreement with and funded by the U.S. Government. Neither the U.S. Government or its employees, nor any of its contractors, subcontractors or their employees, makes any express or implied:

1. warranty or assumes any legal liability for the accuracy, completeness, or for the use or results of such use of any information, product, or process disclosed; or

2. representation that such use or results of such use would not infringe privately owned rights; or

3. endorsement or recommendation of any specifically identified commercial product, process, or service.

Any views and opinions of authors expressed in this work do not necessarily state or reflect those of the United States Government, or its contractors, or subcontractors.

\section{Printed in the United States of America \\ Prepared for U.S. Department of Energy}


Keywords: Performance Assessment PORFLOW

Retention: Permanent

\section{Interpretation of Vadose Zone Monitoring System Data near Engineered Trench 1}

G. P. Flach

T. S. Whiteside

December 12, 2016

Prepared for the U.S. Department of Energy under contract number DE-AC09-08SR22470. 


\section{REVIEWS AND APPROVALS}

\section{AUTHORS:}

TECHNICAL REVIEW:

T. L. Danielson, Environmental Modeling, Reviewed per E7 2.60

APPROVAL:

D. A. Crowley, Manager, Environmental Modeling

Date

K. M. Kostelnik, Director, Environmental Restoration Technology

Date

F. L. Fox, Manager, Solid Waste Programs

Date 
SRNL-STI-2016-00546

Revision 0

\section{ACKNOWLEDGEMENTS}

Gene Shine contributed valuable statistical insights and a Box-Cox analysis of the "Max_high” dataset. 


\section{EXECUTIVE SUMMARY}

The E-Area Vadose Zone Monitoring System (VZMS) includes lysimeter sampling points at many locations alongside and angling beneath the Engineered Trench \#1 (ET1) disposal unit footprint. The sampling points for ET1 were selected for this study because collectively they showed consistently higher tritium (H-3) concentrations than lysimeters associated with other trench units. The VZMS tritium dataset for ET1 from 2001 through 2015 comprises concentrations at or near background levels at approximately half of locations through time, concentrations up to about $600 \mathrm{pCi} / \mathrm{mL}$ at a few locations, and concentrations at two locations that have exceeded $1000 \mathrm{pCi} / \mathrm{mL}$. The highest three values through 2015 were $6472 \mathrm{pCi} / \mathrm{mL}$ in 2014 and $4533 \mathrm{pCi} / \mathrm{mL}$ in 2013 at location VL-17, and $3152 \mathrm{pCi} / \mathrm{mL}$ in 2007 at location VL-15. As a point of reference, the drinking water standard for tritium and a DOE Order 435.1 performance objective in the saturated zone at the distant 100 -meter facility perimeter is $20 \mathrm{pCi} / \mathrm{mL}$. The purpose of this study is to assess whether these elevated concentrations are indicative of a general trend that could challenge 2008 E-Area Performance Assessment (PA) conclusions, or are isolated perturbations that when considered in the context of an entire disposal unit would support PA conclusions.

To this end multiple statistical analyses of ET1 VZMS data were performed herein to estimate the most likely average tritium concentration at shallow depths in the vadose zone (just beneath the trench excavation), and uncertainty in these best-estimates. Median values of VZMS data were then compared to simulated vadose concentrations from the 2008 PA model, a revised model accounting for B-25 box disposal in ET1, and a second revised model that accounted for B-25 boxes and removed a potential model bias in predicted soil moisture content. The 2008 PA model appears to underpredict tritium concentrations observed in the shallow vadose zone. However, data to model comparisons produced little evidence to support a hypothesis that the simulated concentrations from the revised B-25 box models are significantly different from the actual plume concentrations. That is, the revised models appear to be reasonably consistent with the field observations at ET1.

The model revised to account for B-25 box disposal predicts a sum-of-fractions (SOF) less than 1.0 based on the as-disposed-of conditions of ET1 and ET2, which were analyzed together in the 2008 PA and here as a disposal unit group. The model revised to account for B-25 box disposal and to remove a potential model bias in predicted water content forecasts an SOF of 1.05 based on 2008 PA assumptions for plume overlap with disposal unit groups adjoining ET1 and 2 (i.e. east and center Slit Trench groups). However, the phased operation of E-Area disposal units will minimize the potential for any inter-disposal unit group plume interaction. With consideration of this as-disposed-of condition, ET1 is deemed unlikely to exceed performance objectives.

Considering the model improvements developed herein and continued acquisition of tritium data from the VZMS, the following actions are recommended:

1) In the upcoming PA revision, the E-Area vadose model should be revised to account for B-25 box and similar containerized waste disposals. Furthermore, the hydraulic properties assigned to the vadose zone should be reassessed and revised if found to produce a significant bias in simulated moisture content.

2) Upon approval of the next PA, tritium administrative limits for action level lysimeters should be revised to reflect concentrations predicted by the revised PA vadose zone model.

3) Solid Waste should consider incorporating existing groundwater monitoring information as part of the PA monitoring program (specific actions to be developed). It is important to keep in mind that compliance with the DOE Order 435.1 performance objectives is assessed in groundwater rather than the vadose zone. 
4) Vadose zone monitoring should continue to be maintained and expanded for trench units because it provides an early indication of trench disposal unit performance relative to PA assumptions and modeling forecasts, and addresses GW monitoring limitations due to the existing Mixed Waste Management Facility tritium plume beneath E-Area. 


\section{TABLE OF CONTENTS}

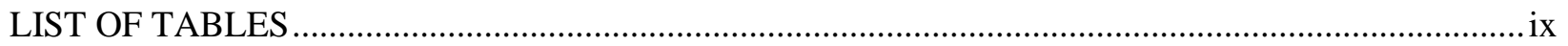

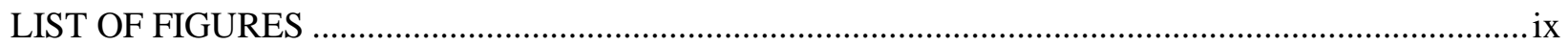

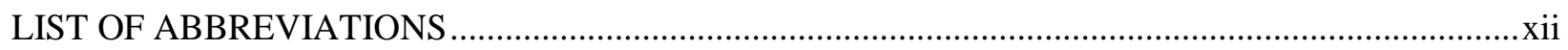

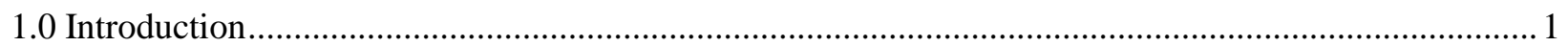

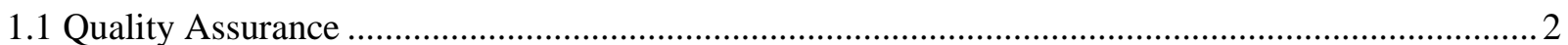

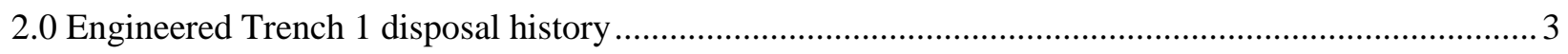

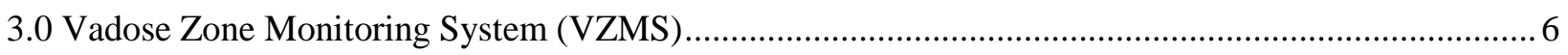

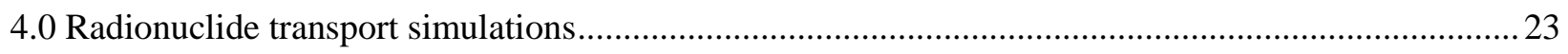

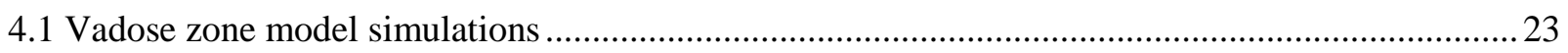

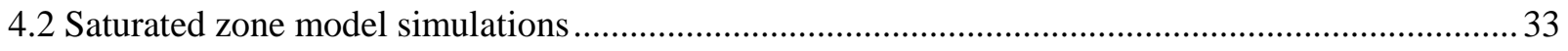

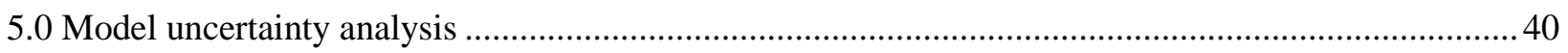

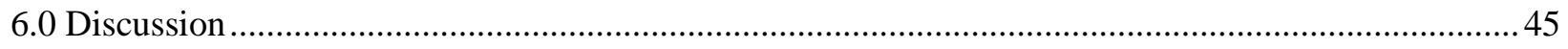

6.1 Observed versus expected tritium concentrations in the vadose zone........................................... 45

6.2 Expectation of meeting DOE 435.1 performance objectives ....................................................... 45

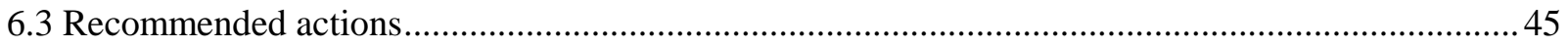

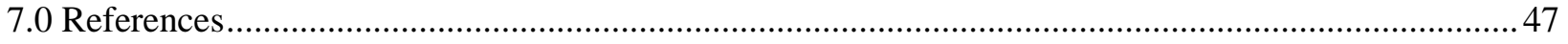

Appendix A . Effective Properties of a Blended Material ........................................................................ 


\section{LIST OF TABLES}

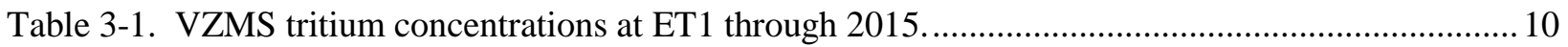

Table 3-2. Estimated initial concentration of tritium in the ET1 waste zone.......................................... 16

Table 3-3. Summary of statistical analysis of VZMS data. ..................................................................... 21

Table 4-1. Material properties of blended backfill soil and low-density boxes...................................... 28

Table 4-2. Simulated peak H-3 concentrations at UPPER and LOWER lysimeter locations.................... 33

Table 4-3. Disposal volume history for ET2 from WITS.................................................................... 34

Table 4-4. Projected WITS sum-of-fractions for aquifer scenarios 0x through 3x................................... 39

Table 5-1. Simulated peak H-3 concentrations at UPPER and LOWER lysimeter locations w/adjusted

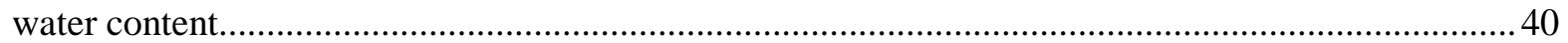

Table 5-2. Projected WITS sum-of-fractions for aquifer scenarios 0x through 3x w/adjusted vadose zone water content.

\section{LIST OF FIGURES}

Figure 2-1. Sum-of-fractions summary for ET1 for the Beta-Gamma 12-100 yr (BG2) pathway.............. 3

Figure 2-2. Tritium (H-3) disposal and volume consumption history for ET1

Figure 2-3. Annotated aerial photo of ET1 from August 2003.............................................................. 4

Figure 2-4. Sequence and approximate timing of waste disposals in ET1 ............................................ 5

Figure 2-5. H-3 activity versus volume consumption in ET1 ............................................................. 5

Figure 3-1. VZMS lysimeter locations and Action Level Lysimeter Exceedances in (a) FY2014 (SRNLSTI-2014-00582) and (b) FY2015 (SRNL-STI-2015-00691) .......................................................... 7

Figure 3-2. Time history of tritium concentrations in ET1 shallow vadose zone VZMS lysimeters. ......... 8

Figure 3-3. Labeled locations of ET1 lysimeters and spatial map of shallow lysimeter concentrations in

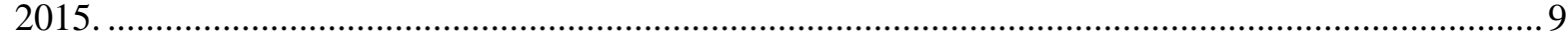

Figure 3-4. Normal probability plots for concentration and log10[concentration] using “Max_high”

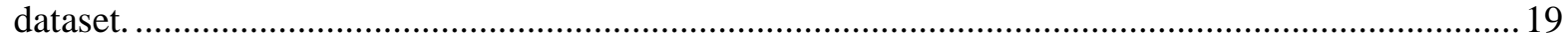

Figure 3-5. Bootstrapping results for the (a) “Max_high” and (b) “2015_high” datasets. ....................... 20

Figure 3-6. Bootstrapping results for FY2015 ET1 Action Level lysimeter dataset. ............................... 22

Figure 4-1. Refined vadose zone grid and added edge and center lysimeter observation points...............24

Figure 4-2. Simulated H-3 plume for Case01 at an elapsed time of 1.0 yr............................................ 24 
Figure 4-3. Simulated vadose zone concentrations for modeling Case01 -- PA + grid refinement + no side slope usage.

Figure 4-4. Simulated vadose zone concentrations for modeling Case02 -- B-25 boxes + no dispersion. 25

Figure 4-5. Simulated vadose zone concentrations for modeling Case03 -- B-25 boxes $+5 \%$ dispersivity. .26

Figure 4-6. Simulated vadose zone concentrations for modeling Case04 -- B-25 boxes $+10 \%$ dispersivity.

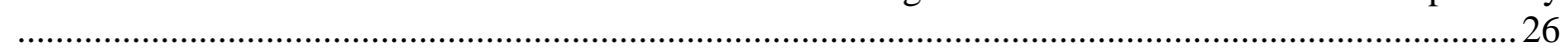

Figure 4-7. Simulated vadose zone concentrations for modeling Case05 -- B25 boxes $+25 \%$ low permeability in undisturbed soil.

Figure 4-8. Simulated vadose zone concentrations for modeling Case06 -- B25 boxes $+35 \%$ low permeability in undisturbed soil. 27

Figure 4-9. Soil characteristics curves for mixed backfill soil and uncompacted boxed waste. 29

Figure 4-10. Simulated H-3 plume for Case02 at an elapsed times of (a) 0.4 yr, (b) 1.0 yr, and (c) 1.6 yr.

Figure 4-11. Random bi-modal hydraulic conductivity fields with low-permeability fractions of (a) $25 \%$ and (b) $35 \%$.

Figure 4-12. Flow fields resulting from low-permeability fractions of (a) 25\% and (b) 35\%.................. 32

Figure 4-13. Simulated tritium concentration for aquifer scenario 0x.................................................. 35

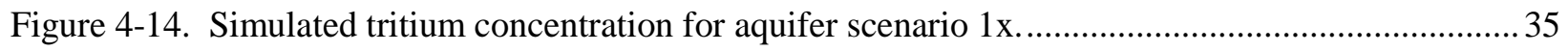

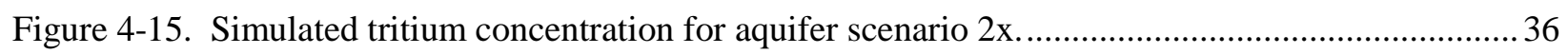

Figure 4-16. Simulated tritium concentration for aquifer scenario 3x.................................................. 36

Figure 4-17. Simulated tracer concentration for aquifer scenario 0x..................................................... 37

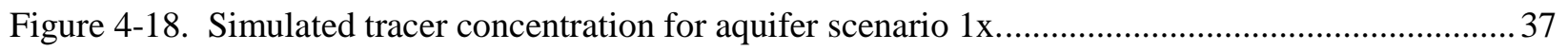

Figure 4-19. Simulated tracer concentration for aquifer scenario 2x.................................................... 38

Figure 4-20. Simulated tracer concentration for aquifer scenario 3x..................................................... 38

Figure 5-1. Simulated vadose zone concentrations for modeling Case01 w/adjusted water content -- PA + grid refinement + no side slope usage. ….................................................................................... 41

Figure 5-2. Simulated vadose zone concentrations for modeling Case02 w/adjusted water content -- B-25

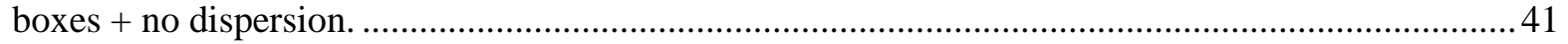

Figure 5-3. Simulated vadose zone concentrations for modeling Case03 w/adjusted water content -- B-25

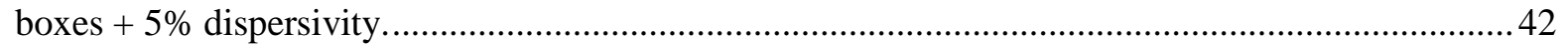

Figure 5-4. Simulated vadose zone concentrations for modeling Case04 w/adjusted water content -- B-25

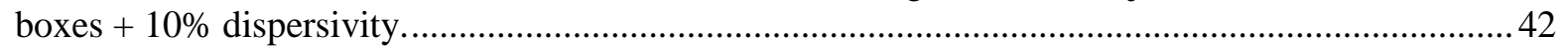


SRNL-STI-2016-00546

Revision 0

Figure 5-5. Simulated vadose zone concentrations for modeling Case05 w/adjusted water content -- B25 boxes $+25 \%$ low permeability in undisturbed soil. ........................................................................... 43

Figure 5-6. Simulated vadose zone concentrations for modeling Case06 w/adjusted water content -- B25 boxes $+35 \%$ low permeability in undisturbed soil. ....................................................................... 43 


\section{LIST OF ABBREVIATIONS}

$\begin{array}{ll}\text { CI } & \text { Confidence Interval } \\ \text { H-3 } & \text { Tritium } \\ \text { ET } & \text { Engineered Trench } \\ \text { OSC } & \text { Operational Soil Cover } \\ \text { PA } & \text { Performance Assessment } \\ \text { SOF } & \text { Sum-of-fractions } \\ \text { SRNL } & \text { Savannah River National Laboratory } \\ \text { VZMS } & \text { Vadose Zone Monitoring System } \\ \text { WITS } & \text { Waste Information Tracking System } \\ \text { WSRC } & \text { Washington Savannah River Company LLC } \\ \text { WT } & \text { Water Table }\end{array}$




\subsection{Introduction}

Solid low-level waste, primarily contained in B-25 boxes, was disposed of in E-Area Engineered Trench (ET) 1 from February 2001 through July 2015 based on Waste Information Tracking System (WITS) records. The radionuclides contributing at least one percentage point (0.01) to the Beta-Gamma 0-12 year (most limiting PA pathway) sum-of-fractions (SOF; WSRC 2008, NRC 2016) in order of decreasing fraction are C-14, I-129, H-3, Tc-99 and Nb-94. As of 9/30/2015 the SOF for these radionuclides of interest was 0.854 compared to a total SOF of 0.868 per WITS.

The E-Area Vadose Zone Monitoring System (VZMS) includes lysimeter sampling points at many locations alongside the ET1 disposal unit footprint. Considering the relative mobility of H-3 compared to other nuclides, VZMS sampling events have focused on tritium analyses, although other species have also been analyzed on occasion. The VZMS tritium dataset for ET1 from 2001 through 2015 comprises concentrations at or near background levels at approximately half of locations through time, concentrations up to about $600 \mathrm{pCi} / \mathrm{mL}$ at a few locations, and concentrations at two locations that have exceeded $1000 \mathrm{pCi} / \mathrm{mL}$. The maximum values through 2015 were $6472 \mathrm{pCi} / \mathrm{mL}$ in 2014 and $4533 \mathrm{pCi} / \mathrm{mL}$ in 2013 at location VL-17, and $3152 \mathrm{pCi} / \mathrm{mL}$ in 2007 at location VL-15. As a point of reference, the drinking water standard for tritium and a performance objective at the 100-meter facility perimeter is 20 $\mathrm{pCi} / \mathrm{mL}$. The purpose of this study is to assess whether these elevated concentrations are indicative of a general trend that could challenge 2008 E-Area Performance Assessment (PA) conclusions, or are isolated perturbations that when considered in the context of a complete disposal unit would not dispute PA conclusions.

ET1 disposal limits are designed to satisfy the performance objectives of DOE Order 435.1 based on a 2008 E-Area Performance Assessment (PA) analysis (WSRC 2008). The E-Area PA predicted the radionuclide flux crossing the water table (WT) and the peak radionuclide concentration in the aquifer underlying E-Area along the facility 100 meter perimeter. Simulated radionuclide concentrations in the vadose zone soil column are not reported in the PA document, precluding direct comparison of VZMS data to published model predictions. Furthermore, the PA analysis assumed that

- a uniform distribution of radionuclide activity occurred within the ET1 footprint,

- that disposals filling ET1 occurred at a single instant,

- the equivalent of backfill soil would fill the entire trench volume at closure, and

- $\quad$ waste containers would not hinder (e.g. delay) waste release.

The as-disposed-of ET1 condition comprises a non-uniform waste distribution, disposals occurring over a nearly 15 year period, significant void space within the waste zone due to disposal of low-density boxed waste, and waste containers that likely affect radionuclide release. These differences constitute a mixture of conservative and non-conservative conditions relative to the 2008 PA.

To enable comparison of VZMS data to model predictions, 2008 E-Area PA models were retrieved from the archives and modified in this study to: 1) capture vadose zone concentrations at locations representative of VZMS lysimeters, and 2) more closely reflect the as-disposed-of condition of ET1 now that the disposal unit has reached $99.5 \%$ volume capacity. The study also includes a statistical analysis of VZMS tritium data to determine a representative (e.g. average) concentration for comparison to model predictions, and assess whether the field observations are consistent with the tritium inventory reported in WITS and PA-based model predictions. Inferences concerning the overarching question of whether ET1 will meet performance objectives are then drawn. 


\subsection{Quality Assurance}

Requirements for performing reviews of technical reports and the extent of review are established in Manual E7 Procedure 2.60. SRNL documents the extent and type of review using the SRNL Technical Report Design Checklist contained in WSRC-IM-2002-00011, Rev. 2. 


\subsection{Engineered Trench 1 disposal history}

Figure 2-1 through Figure 2-3 contain key disposal history information provided by Solid Waste Engineering. Although the SOF for ET1 is less than one (0.868) indicating remaining radionuclide disposal capacity, the disposal unit is practically full from a volume capacity perspective (99.5\%). Disposal unit closure is anticipated in FY2017. Figure 2-4 illustrates the sequence and approximate timing of ET1 waste disposals inferred from Figure 2-2 and Figure 2-3. Figure 2-5 compares disposed H3 activity to volume consumption as percentages of their totals and indicates an overall distribution of tritium throughout the trench. The most limiting PA pathway is Beta-Gamma 12-100 years (BG2) and the disposal limit for this pathway for H-3 is 12 Ci (Swingle 2012a, Table 20, Swingle 2012b). The disposed $\mathrm{H}-3$ activity is $2.21 \mathrm{Ci}$ as of September 2015, which is the dataset used by this study (Figure 2-2).

\begin{tabular}{|c|c|}
\hline ET\#1 BG2 (12 - 100 & OF for Key \\
\hline & (as of $9 / 30$ \\
\hline Isotope & SOF in BG2 \\
\hline C14 & 0.262 \\
\hline H3 & 0.185 \\
\hline 1129 & 0.245 \\
\hline NB94 & 0.017 \\
\hline TC99 & 0.145 \\
\hline Sum Total & 0.854 \\
\hline Overa & \\
\hline SOF & 0.868 \\
\hline Total & \\
\hline
\end{tabular}

Figure 2-1. Sum-of-fractions summary for ET1 for the Beta-Gamma 12-100 yr (BG2) pathway. 


\begin{tabular}{|c|c|c|c|c|c|c|c|c|c|c|c|c|c|}
\hline \multicolumn{14}{|c|}{ ET\#1 Volume Status and Cumulative H3 Disposed Inventory } \\
\hline Month & Jul-15 & Jan-15 & Jul-14 & Jan-14 & Jul-13 & Jan-13 & Jul-12 & Jan-12 & Jul-11 & Jan-11 & Jul-10 & Jan-10 & Jul-09 \\
\hline & & & & & & & & & & & & & \\
\hline Vol (\% full) & $99.5 \%$ & $99.0 \%$ & $98.7 \%$ & $98.7 \%$ & $98.7 \%$ & $98.7 \%$ & $98.7 \%$ & $98.7 \%$ & $98.7 \%$ & $98.7 \%$ & $88.4 \%$ & $88.4 \%$ & $88.4 \%$ \\
\hline H-3 Disposed (Ci) - Cumulative & $2.21 \mathrm{E}+00$ & $2.21 \mathrm{E}+00$ & $2.21 \mathrm{E}+00$ & \begin{tabular}{|l|l|l|l|l}
$2.21 \mathrm{E}+00$ & 2 \\
\end{tabular} & $2.21 \mathrm{E}+00$ & $2.21 \mathrm{E}+00$ & $2.21 \mathrm{E}+00$ & $2.21 \mathrm{E}+00$ & $2.21 \mathrm{E}+00$ & $2.21 \mathrm{E}+00$ & $1.69 \mathrm{E}+00$ & $1.69 \mathrm{E}+00$ & $1.69 \mathrm{E}+00$ \\
\hline Month & Feb-09 & Jul-08 & Jan-08 & Jul-07 & Jan-07 & Jul-06 & Jan-06 & Jul-05 & Jan-05 & Jul-04 & Jan-04 & Jul-03 & Jan-03 \\
\hline Vol (\% full) & $88.4 \%$ & $88.4 \%$ & $88.4 \%$ & $88.4 \%$ & $88.4 \%$ & $87.8 \%$ & $83.5 \%$ & $79.2 \%$ & $74.3 \%$ & $73.2 \%$ & $69.3 \%$ & $57.6 \%$ & $45.9 \%$ \\
\hline H-3 Disposed (Ci) - Cumulative & $1.69 \mathrm{E}+00$ & $1.69 \mathrm{E}+00$ & $1.69 E+00$ & \begin{tabular}{|l|l}
$1.69 \mathrm{E}+00$ & 1 \\
\end{tabular} & $1.69 \mathrm{E}+00$ & $1.69 \mathrm{E}+00$ & $1.69 \mathrm{E}+00$ & $1.69 \mathrm{E}+00$ & $1.69 \mathrm{E}+00$ & $1.68 \mathrm{E}+00$ & $1.65 \mathrm{E}+00$ & $1.30 \mathrm{E}+00$ & $9.59 \mathrm{E}-01$ \\
\hline Month & Jul-02 & Jan-02 & Jul-01 & Feb-01 & & & & & & & & & \\
\hline Vol (\% full) & $24.7 \%$ & $18.0 \%$ & $8.1 \%$ & $0.0 \%$ & & & & & & & & & \\
\hline H-3 Disposed (Ci) - Cumulative & $3.19 \mathrm{E}-01$ & $2.64 \mathrm{E}-01$ & $8.17 \mathrm{E}-02$ & $0.00 \mathrm{E}+00$ & & & & & & & & & \\
\hline
\end{tabular}

Figure 2-2. Tritium (H-3) disposal and volume consumption history for ET1.

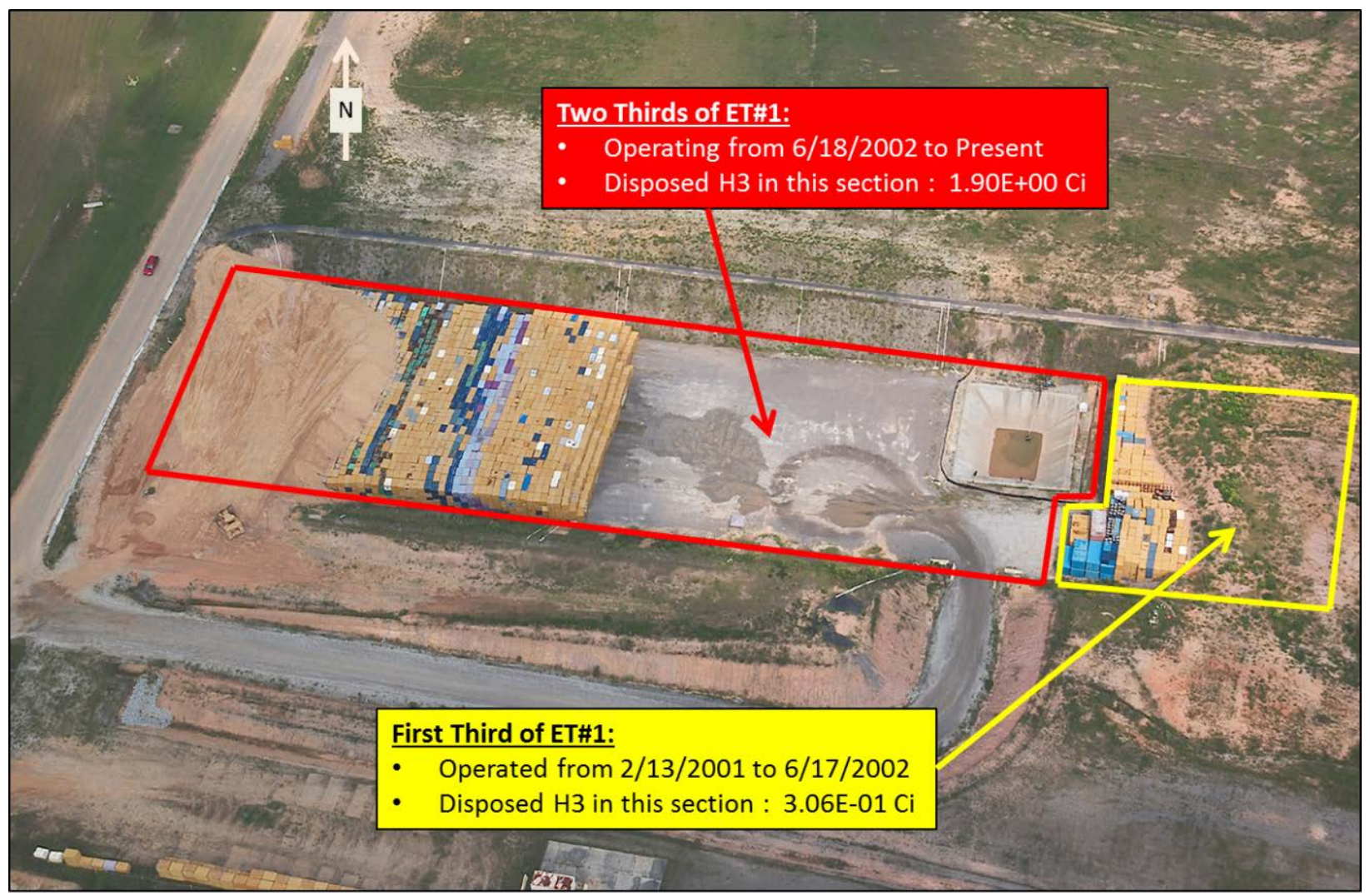

Figure 2-3. Annotated aerial photo of ET1 from August 2003. 


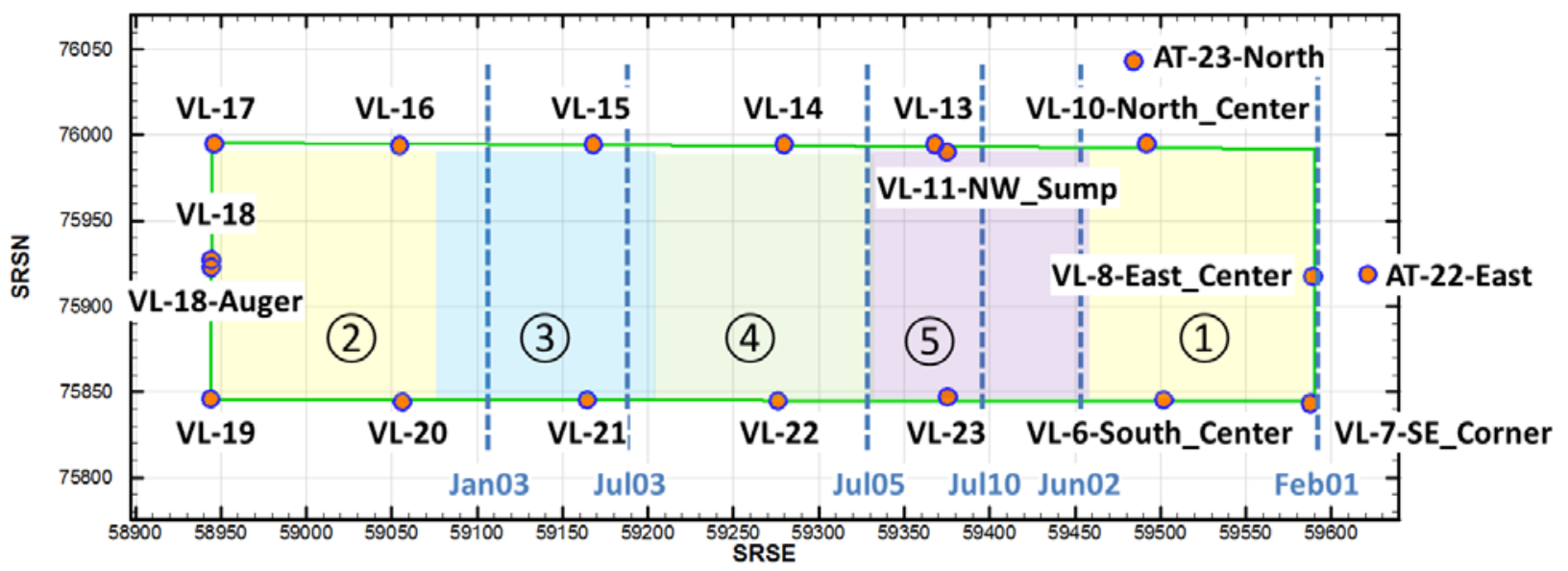

Key:

- $\quad$ Shaded blocks represent $20 \%$ of disposal volume and (1) $\rightarrow$ (5) indicates sequence of disposals

- Dashed lines and month/year indicate approximate position of disposal progress

- Points represent ground surface locations of lysimeters

Figure 2-4. Sequence and approximate timing of waste disposals in ET1.

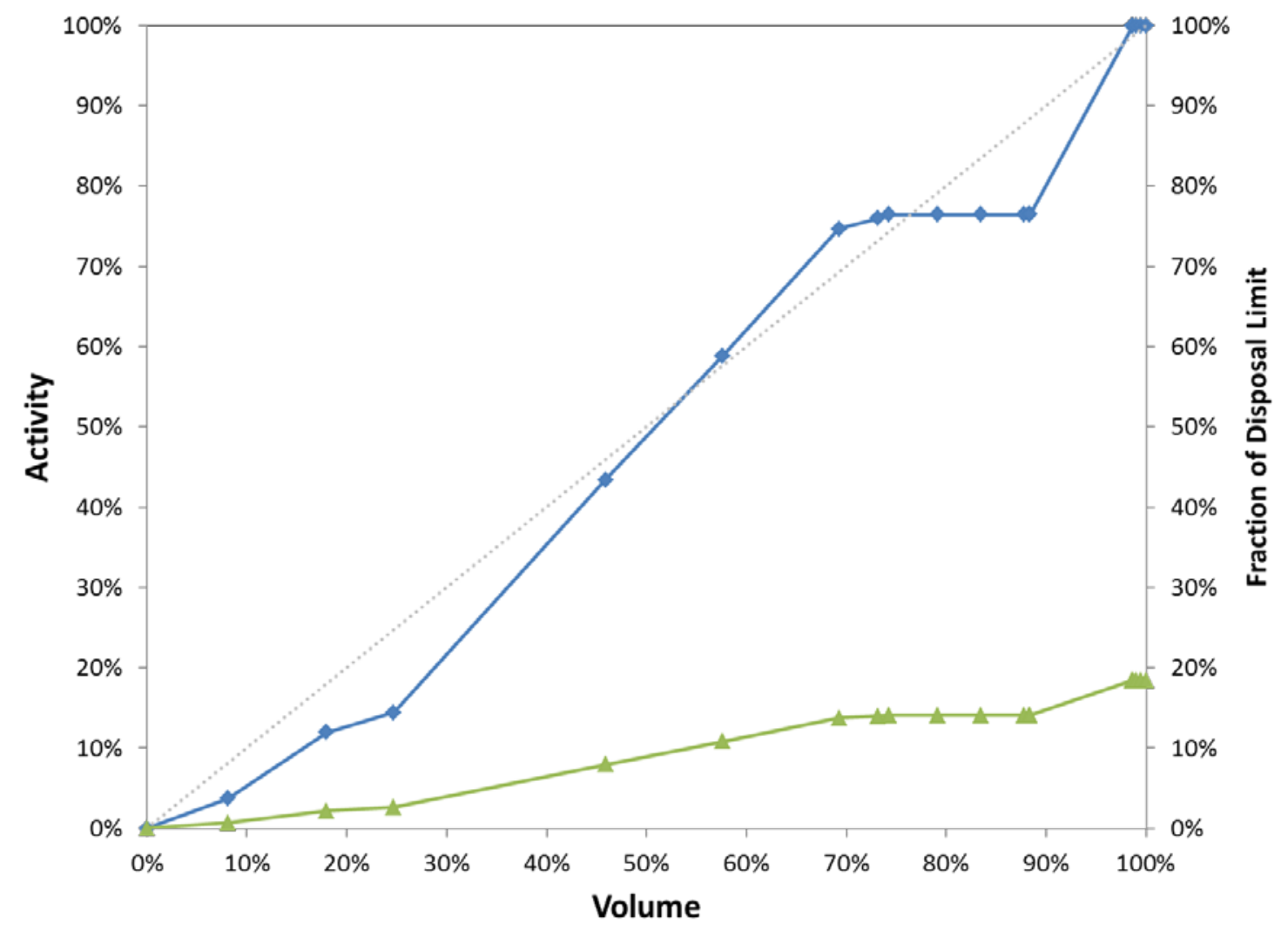

Figure 2-5. H-3 activity versus volume consumption in ET1. 


\subsection{Vadose Zone Monitoring System (VZMS)}

The Vadose Zone Monitoring System (WSRC 2008, Millings 2009, 2012, Hiergesell et al. 2015, 2016) is a collection of lysimeters that have been placed alongside and beneath the footprint of ET1 and other EArea trench disposal units. Soil moisture samples are drawn from the system twice annually and analyzed for tritium. The objectives of the VZMS are to: “1) monitor trends in performance, 2) evaluate whether a facility is operating and behaving as expected and predicted by the PA, 3) evaluate the conservativeness of the PA conclusions, 4) provide input for refining the PA and building integrity in the PA analyses, and 5) provide a means to evaluate the potential for future regulatory exceedances” (Hiergesell et al. 2015).

Figure 3-1 identifies the locations of single lysimeters and multi-depth clusters, and those Action Level lysimeters that exceeded administrative limits for tritium in FY2014 and FY2015. The administrative limit is set to $25 \%$ of the average tritium concentration that would occur in the deep vadose zone for a trench operating at its radiological disposal limit for tritium. The administrative limit for Engineered Trenches is $101 \mathrm{pCi} / \mathrm{mL}$ (Hiergesell et al. 2016, Table 5-1). Exceedance of the administrative limit in a deep vadose zone lysimeter triggers further study to assess whether the disposal unit is performing as expected based on the E-Area Performance Assessment (WSRC 2008). At ET1, a single administrative limit was exceeded in FY2014 and three in FY2015. Further information is provided in Table 5-2 of both Hiergesell et al. (2015) and (2016). 


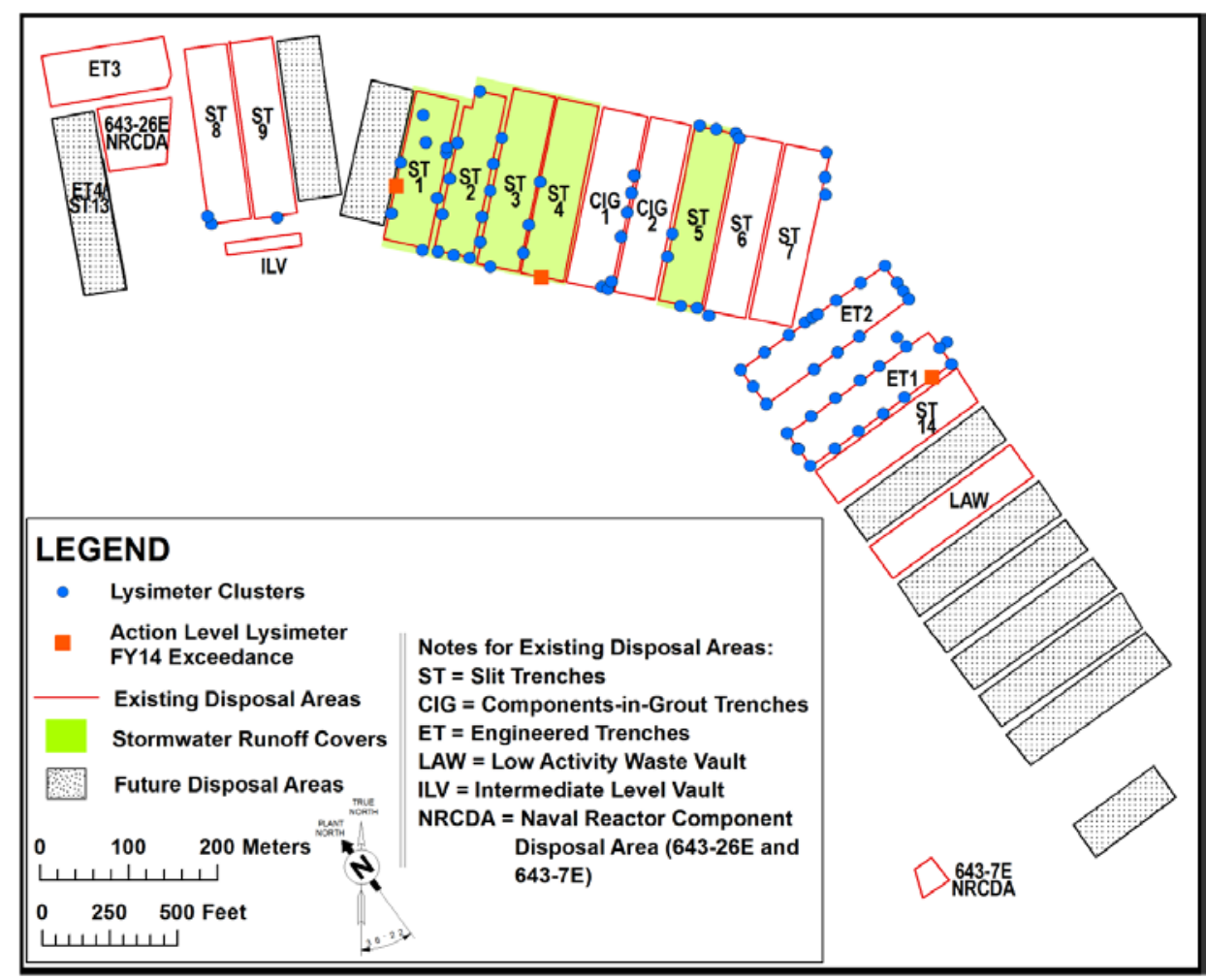

(a)

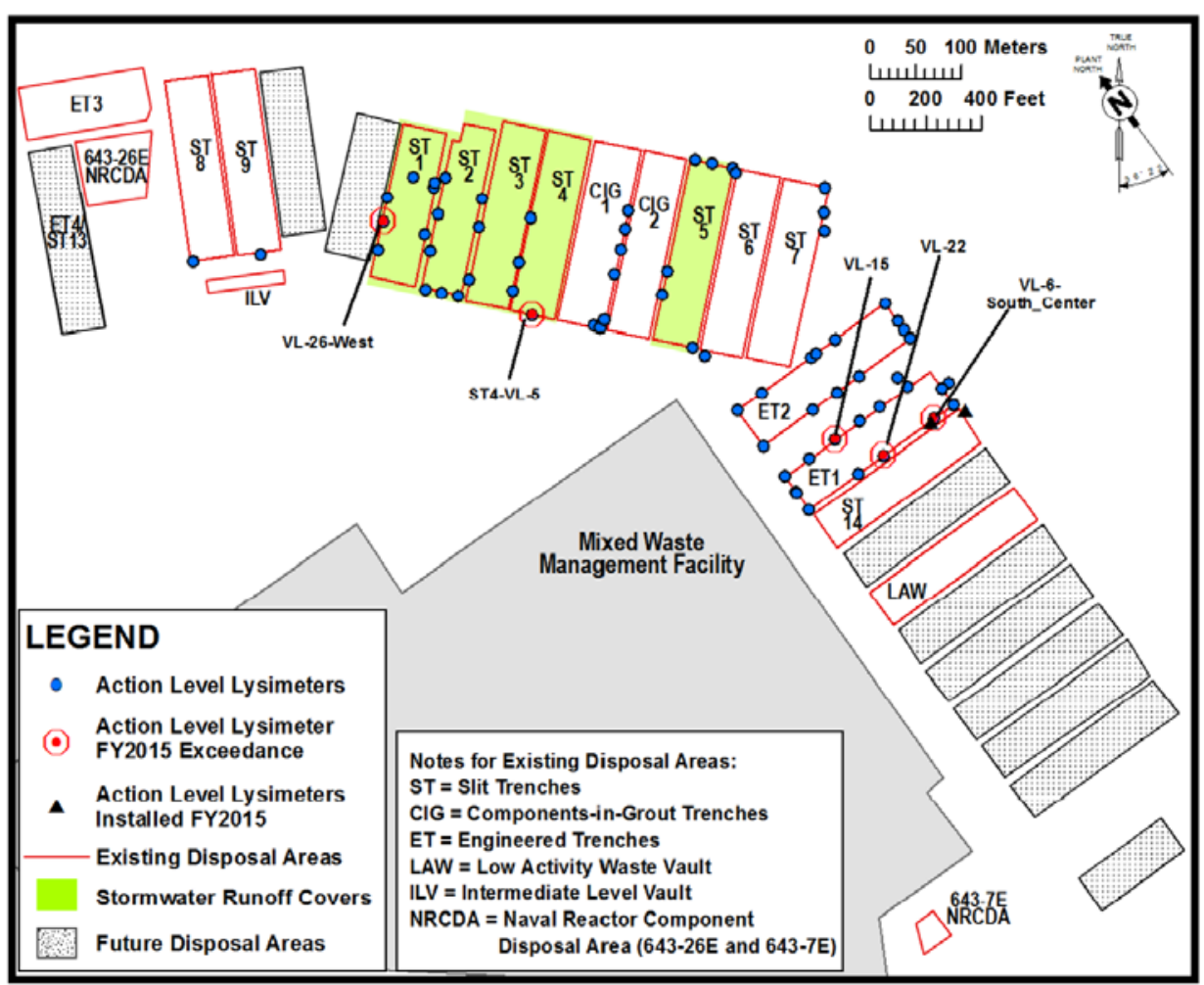

(b)

Figure 3-1. VZMS lysimeter locations and Action Level Lysimeter Exceedances in (a) FY2014 (SRNL-STI-2014-00582) and (b) FY2015 (SRNL-STI-2015-00691). 
Shown in Figure 3-2 are tritium concentrations from the shallow-most lysimeter at each ET1 location, generally a few feet below the trench bottom, as a function of time. The shallow-most lysimeter generally produces the highest concentration at a particular location, but not always. Approximately half of the shallow lysimeters have produced tritium concentrations in vadose zone soil moisture exceeding the drinking water standard $(20 \mathrm{pCi} / \mathrm{mL})$ for at least one sampling event. The drinking water standard is used here as a point of reference and is not a performance objective for tritium in vadose zone soil moisture. Table 3-1 lists the maximum VZMS concentration for each sampling year through 2015. Lysimeters are generally sampled twice per year. For each year Table 3-1 lists the peak concentration recorded for that calendar year. A blank entry for a location indicates that no data were acquired for that calendar year, because the lysimeter was abandoned, not sampled, or insufficient sample volume was obtained for analysis.

As noted earlier, the highest three concentrations through 2015 were $6472 \mathrm{pCi} / \mathrm{mL}$ (VL-17, 2014), 4533 $\mathrm{pCi} / \mathrm{mL}$ (VL-17, 2013) and $3152 \mathrm{pCi} / \mathrm{mL}$ (VL-15, 2007). The three locations exhibiting the high concentrations at any point in time are VL-17, VL-15 and VL-6-South_Center. Figure 3-3 illustrates the spatial distribution of tritium concentrations from shallow lysimeters in 2015 based on kriging interpolation of sample data across the trench footprint. No data were available from lysimeter VL-10North_Center in 2015, and for the purpose of kriging interpolation, VL-11_NW_Sump data were omitted in favor of nearly co-located VL-13 data, and data from the AT lysimeter was repositioned to the perimeter of ET1.

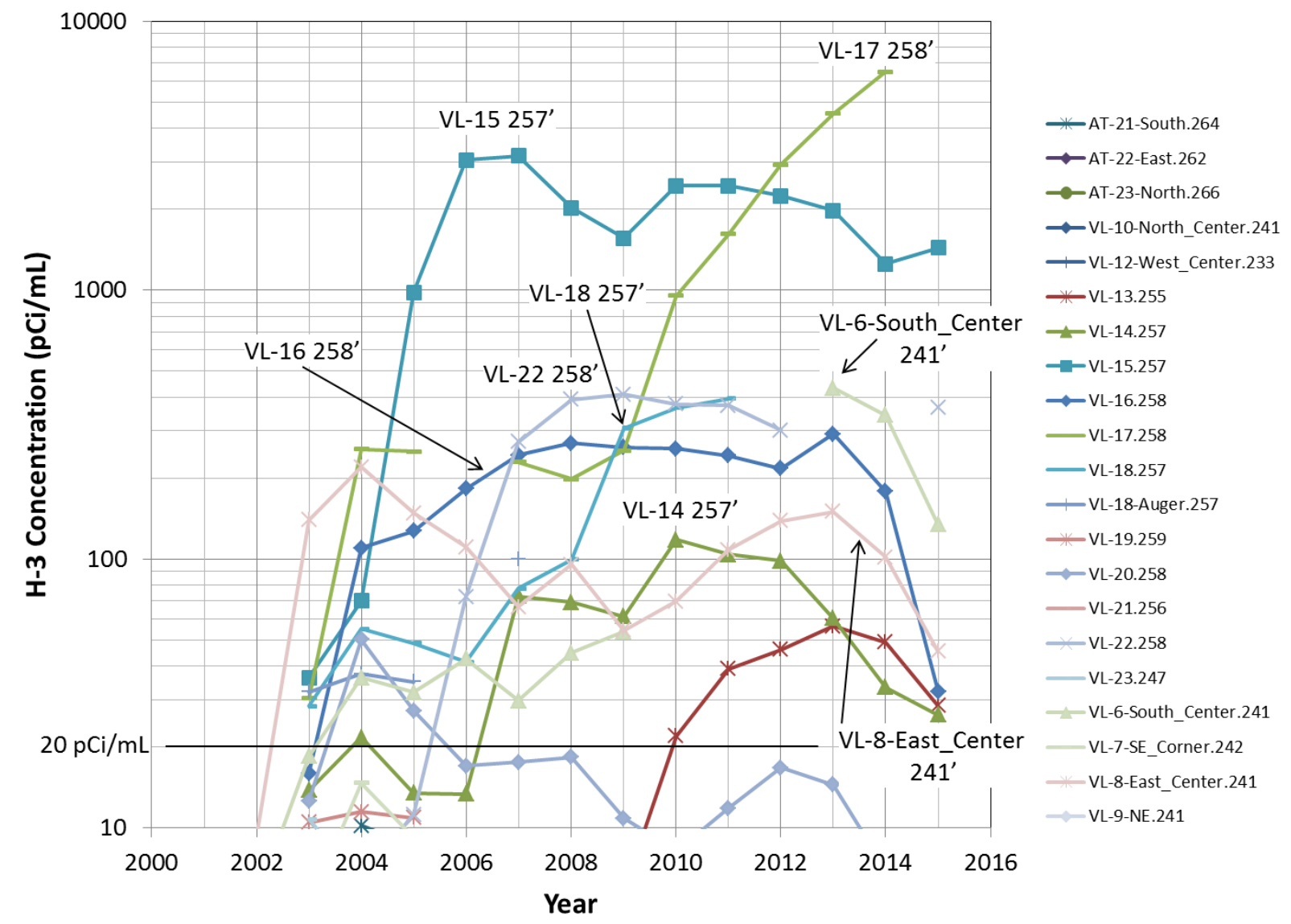

Figure 3-2. Time history of tritium concentrations in ET1 shallow vadose zone VZMS lysimeters. 


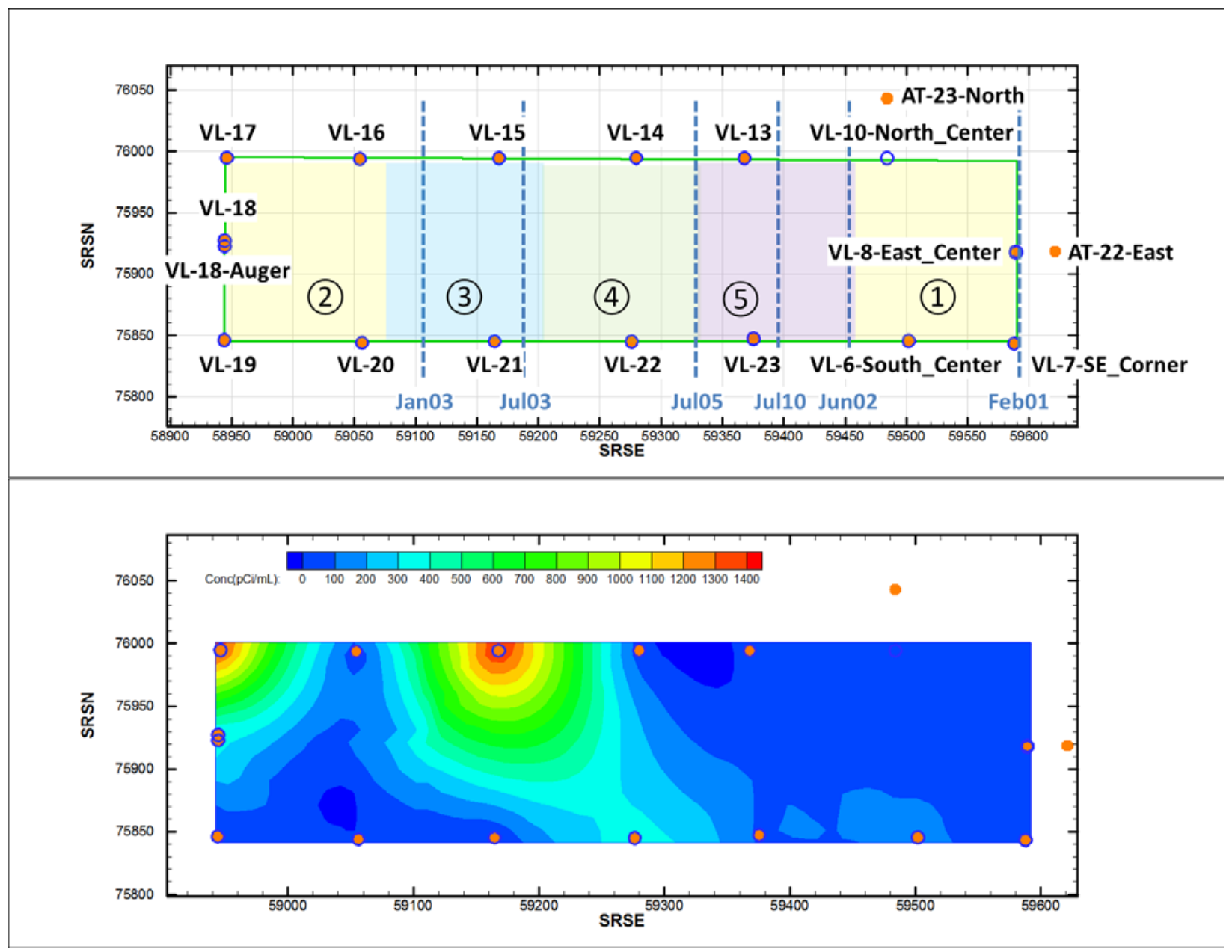

Figure 3-3. Labeled locations of ET1 lysimeters and spatial map of shallow lysimeter concentrations in 2015. 
Table 3-1. VZMS tritium concentrations at ET1 through 2015.

\begin{tabular}{|c|c|c|c|c|c|c|c|c|c|c|c|c|c|c|c|c|}
\hline H-3 Conc. [pCi/mL] & 2001 & 2002 & 2003 & 2004 & 2005 & 2006 & 2007 & 2008 & 2009 & 2010 & 2011 & 2012 & 2013 & 2014 & 2015 & $\begin{array}{l}\text { Max. } \\
\text { conc. }\end{array}$ \\
\hline AT-21-South & 8.5 & 9.5 & 8.6 & 11.9 & 11.0 & 10.7 & 8.9 & 7.3 & 6.3 & & 5.7 & 6.1 & & & & 11.9 \\
\hline 235 & 8.5 & 9.5 & 8.6 & 9.0 & 8.5 & 7.9 & 7.0 & 7.3 & 6.3 & & 5.7 & 6.1 & & & & 9.5 \\
\hline 240 & & & & 9.3 & 7.4 & & & & & & & & & & & 9.3 \\
\hline 255 & & & & 11.9 & 11.0 & 10.7 & 8.9 & & & & & & & & & 11.9 \\
\hline 264 & & & & 10.2 & 8.6 & 8.1 & & & & & & & & & & 10.2 \\
\hline AT-22-East & 10.1 & 11.3 & 9.5 & 10.7 & 7.9 & 8.6 & 6.6 & 6.9 & 6.3 & 6.7 & 6.3 & 6.1 & 6.7 & 5.8 & 4.8 & 11.3 \\
\hline $233(\mathrm{AL})$ & 9.4 & 8.6 & 8.6 & 8.6 & 7.5 & 6.5 & 6.6 & 6.6 & 6.3 & 6.7 & 6.3 & 6.1 & 6.7 & 5.8 & 4.8 & 9.4 \\
\hline 238 & 9.0 & 8.7 & 8.8 & 8.6 & 7.7 & 8.6 & 6.3 & 6.9 & 5.3 & 4.9 & 4.7 & 4.4 & 4.7 & 4.1 & 3.5 & 9.0 \\
\hline 253 & 10.1 & 11.3 & 9.5 & 10.7 & 6.6 & 5.4 & & 5.1 & 4.7 & 4.5 & 4.6 & 4.5 & 4.6 & 4.0 & 3.9 & 11.3 \\
\hline 262 & 9.2 & 9.4 & 9.1 & 9.0 & 7.9 & 6.7 & 5.8 & 5.6 & & & & & & & & 9.4 \\
\hline AT-23-North & 6.7 & 6.1 & 5.8 & 10.6 & 7.6 & 7.0 & 5.1 & 4.2 & 3.5 & 3.3 & 3.7 & 3.8 & 3.5 & 3.5 & 3.2 & 10.6 \\
\hline $237(\mathrm{AL})$ & 5.5 & 5.8 & 5.8 & 10.6 & 4.8 & 4.4 & 4.5 & 4.2 & 3.5 & 3.3 & 3.1 & 3.3 & 3.3 & 3.5 & 3.2 & 10.6 \\
\hline 242 & 6.7 & 6.1 & 5.6 & 5.9 & 5.3 & 4.1 & 4.0 & 3.7 & 3.4 & 3.3 & 3.7 & 3.8 & 3.5 & 3.2 & 2.7 & 6.7 \\
\hline 257 & & & & 6.3 & 6.5 & 5.9 & 5.1 & & & & & & & & & 6.5 \\
\hline 266 & & & & 9.1 & 7.6 & 7.0 & & & & & & & & & & 9.1 \\
\hline VL-10-North_Center & 4.5 & 7.2 & 6.4 & 8.5 & 7.2 & 7.4 & 6.8 & 6.3 & 5.1 & 5.2 & 4.7 & 3.9 & 4.9 & & & 8.5 \\
\hline 219 & & & & 5.5 & & & 0.5 & & & & & & & & & 5.5 \\
\hline $233(\mathrm{AL})$ & 4.1 & 7.2 & 6.4 & 6.3 & 7.0 & 7.4 & 6.8 & 6.3 & 5.1 & 5.2 & 4.7 & 3.9 & 4.9 & & & 7.4 \\
\hline 241 & 4.5 & 6.2 & 5.9 & 8.5 & 7.2 & 6.7 & 6.7 & & & & & & & & & 8.5 \\
\hline VL-11-NW_Sump & 6.5 & 6.5 & 3.6 & 4.7 & 6.0 & & 4.5 & 9.3 & 10.3 & 6.7 & & & & & & 10.3 \\
\hline 220 & 6.5 & 6.5 & 3.6 & 4.7 & 6.0 & & 4.2 & 9.3 & 10.3 & 6.7 & & & & & & 10.3 \\
\hline 241 & & & & & & & 4.5 & & & & & & & & & 4.5 \\
\hline VL-12-West_Center & 8.4 & 6.6 & 4.8 & 11.1 & 4.7 & 4.5 & & & & & & & & & & 11.1 \\
\hline 219 & & & & 11.1 & & & & & & & & & & & & 11.1 \\
\hline 233 & 8.4 & 6.6 & 4.8 & 3.9 & 4.7 & 4.5 & & & & & & & & & & 8.4 \\
\hline VL-13 & & & 9.0 & 8.2 & 6.2 & 6.5 & 20.6 & 4.7 & 8.2 & 22.0 & 39.0 & 46.1 & 56.4 & 89.5 & 28.7 & 89.5 \\
\hline $229(\mathrm{AL})$ & & & 9.0 & 8.2 & 4.2 & 2.6 & 2.7 & 3.3 & 3.1 & 3.1 & 3.6 & 4.0 & 5.6 & 7.1 & 7.1 & 9.0 \\
\hline
\end{tabular}


SRNL-STI-2016-00546

Revision 0

\begin{tabular}{|c|c|c|c|c|c|c|c|c|c|c|c|c|c|c|c|c|}
\hline H-3 Conc. [pCi/mL] & 2001 & 2002 & 2003 & 2004 & 2005 & 2006 & 2007 & 2008 & 2009 & 2010 & 2011 & 2012 & 2013 & 2014 & 2015 & $\begin{array}{l}\text { Max. } \\
\text { conc. }\end{array}$ \\
\hline 237 & & & 4.3 & 5.3 & 3.5 & 4.8 & 4.5 & 4.3 & 8.2 & & 8.2 & 26.1 & 20.2 & 89.5 & 28.7 & 89.5 \\
\hline 244 & & & 5.9 & 6.1 & 4.5 & & & & & & & & & & & 6.1 \\
\hline 247 & & & 5.9 & 5.3 & 4.5 & 5.2 & 20.6 & 4.7 & 4.7 & 4.9 & 9.5 & 15.1 & 35.5 & 34.8 & 13.9 & 35.5 \\
\hline 254 & & & 6.3 & 4.2 & 5.7 & 5.4 & 7.1 & & 6.2 & 10.7 & 23.5 & 17.9 & 26.3 & & 20.0 & 26.3 \\
\hline 255 & & & 4.8 & 5.4 & 6.2 & 6.5 & 7.7 & 4.7 & 5.8 & 22.0 & 39.0 & 46.1 & 56.4 & 49.0 & 28.5 & 56.4 \\
\hline VL-14 & & & 13.8 & 21.7 & 13.5 & 45.6 & 73.1 & 69.1 & 61.1 & 118.0 & 115.1 & 98.3 & 60.2 & 77.4 & 62.7 & 118.0 \\
\hline 229 & & & 6.1 & 5.6 & 5.8 & & & & & & & & & & & 6.1 \\
\hline $239(\mathrm{AL})$ & & & 6.3 & 8.2 & 7.9 & 8.3 & 8.5 & 11.4 & 11.1 & 11.2 & 10.2 & 11.1 & 20.5 & 53.0 & 49.5 & 53.0 \\
\hline 246 & & & 8.3 & 8.3 & 8.6 & 31.2 & 73.1 & 20.3 & 18.2 & 18.0 & 26.0 & 31.7 & 44.9 & 49.1 & 45.0 & 73.1 \\
\hline 251 & & & 9.4 & 9.9 & 10.4 & 11.0 & 25.2 & 20.4 & 19.5 & 25.9 & 115.1 & 47.0 & 55.0 & 77.4 & 62.7 & 115.1 \\
\hline 255 & & & 9.6 & 15.6 & 9.9 & 45.6 & 29.9 & 32.1 & 37.9 & 38.0 & 47.1 & 61.3 & 40.6 & 51.8 & 26.2 & 61.3 \\
\hline 257 & & & 13.8 & 21.7 & 13.5 & 13.4 & 72.8 & 69.1 & 61.1 & 118.0 & 104.1 & 98.3 & 60.2 & 33.3 & 26.4 & 118.0 \\
\hline VL-15 & & & 36.2 & 69.8 & 1003.0 & 3040.0 & 3151.8 & 2051.7 & 1831.0 & 2447.7 & 2445.5 & 2387.3 & 2128.0 & 1467.9 & 1434.5 & 3151.8 \\
\hline 228 (AL) & & & 6.2 & 4.5 & 5.6 & 6.4 & 4.3 & 5.7 & 5.3 & 6.7 & 10.7 & & & 26.2 & & 26.2 \\
\hline 235 & & & 3.8 & 4.4 & 7.7 & 17.9 & 23.1 & 27.3 & & 39.9 & 90.6 & 204.0 & & 704.6 & 1057.9 & 1057.9 \\
\hline 241 & & & 4.7 & 47.4 & 52.3 & 49.4 & 196.5 & 450.2 & 558.1 & 726.6 & 1376.2 & 1577.4 & 1454.9 & 993.7 & 722.1 & 1577.4 \\
\hline 244 & & & 6.1 & 55.4 & 52.8 & 123.0 & 409.8 & 618.2 & 763.0 & 1098.7 & 1366.9 & 2387.3 & 1576.5 & 1467.9 & 446.4 & 2387.3 \\
\hline 251 & & & 24.7 & 53.7 & & & & & & & & & & & & 53.7 \\
\hline 255 & & & 33.8 & 66.6 & 1003.0 & 1930.0 & 2594.9 & 2051.7 & 1831.0 & 2358.1 & 2370.4 & 2273.2 & 2128.0 & 1063.0 & 1174.0 & 2594.9 \\
\hline 257 & & & 36.2 & 69.8 & 980.0 & 3040.0 & 3151.8 & 2026.0 & 1558.4 & 2447.7 & 2445.5 & 2242.8 & 1977.8 & 1252.9 & 1434.5 & 3151.8 \\
\hline VL-16 & & & 97.0 & 110.0 & 127.0 & 183.0 & 243.2 & 270.1 & 259.9 & 257.5 & 242.6 & 217.5 & 292.3 & 214.9 & 60.0 & 292.3 \\
\hline 229 & & & 97.0 & 57.7 & 20.3 & 26.4 & 11.3 & & & & & & & & & 97.0 \\
\hline 235 (AL) & & & 8.0 & 13.9 & 8.2 & 8.0 & 8.6 & 8.4 & 8.4 & 8.8 & 8.7 & 9.4 & 8.5 & 8.1 & 8.4 & 13.9 \\
\hline 241 & & & 8.9 & 11.0 & 10.9 & 10.7 & 11.5 & 11.1 & 11.3 & 11.4 & 11.4 & 14.8 & 15.3 & 13.4 & 13.9 & 15.3 \\
\hline 247 & & & 12.1 & 15.4 & 16.9 & 17.6 & 15.2 & 14.5 & 16.5 & 23.4 & 19.6 & 14.9 & 17.4 & 14.4 & 10.4 & 23.4 \\
\hline 251 & & & 14.0 & 23.4 & 23.1 & 25.6 & 31.8 & 40.7 & 43.6 & 28.1 & 20.4 & 41.9 & 25.0 & 24.5 & 19.1 & 43.6 \\
\hline 255 & & & 15.7 & 49.5 & 95.6 & 140.0 & 144.2 & 148.6 & 152.6 & 149.2 & 146.5 & 144.4 & 146.6 & 214.9 & 60.0 & 214.9 \\
\hline 258 & & & 16.0 & 110.0 & 127.0 & 183.0 & 243.2 & 270.1 & 259.9 & 257.5 & 242.6 & 217.5 & 292.3 & 178.1 & 32.2 & 292.3 \\
\hline
\end{tabular}




\begin{tabular}{|c|c|c|c|c|c|c|c|c|c|c|c|c|c|c|c|c|}
\hline H-3 Conc. [pCi/mL] & 2001 & 2002 & 2003 & 2004 & 2005 & 2006 & 2007 & 2008 & 2009 & 2010 & 2011 & 2012 & 2013 & 2014 & 2015 & $\begin{array}{l}\text { Max. } \\
\text { conc. }\end{array}$ \\
\hline VL-17 & & & 30.3 & 256.0 & 251.0 & 177.0 & 229.7 & 215.9 & 264.0 & 955.2 & 1618.7 & 2932.8 & 4533.0 & 6472.1 & 1387.7 & 6472.1 \\
\hline $231(\mathrm{AL})$ & & & 8.4 & 17.9 & 10.5 & 10.2 & 11.3 & 9.3 & 9.3 & 9.2 & 8.6 & 10.7 & 10.2 & 14.4 & 11.2 & 17.9 \\
\hline 238 & & & 9.7 & 10.9 & 11.6 & 11.4 & 11.7 & 11.2 & 10.7 & 12.2 & 11.2 & 15.1 & 17.8 & 19.5 & 25.3 & 25.3 \\
\hline 243 & & & 9.1 & & & & & & & & & & & & & 9.1 \\
\hline 247 & & & 10.0 & 18.9 & 17.7 & 18.3 & 22.9 & 32.0 & 58.9 & 65.6 & 69.8 & 199.6 & 247.1 & 255.7 & 202.4 & 255.7 \\
\hline 251 & & & 11.3 & 25.9 & 42.9 & & 82.3 & & & & & & & & & 82.3 \\
\hline 256 & & & 16.3 & 104.0 & 146.0 & 177.0 & 199.9 & 215.9 & 264.0 & 259.0 & 277.3 & 483.1 & 1043.3 & 1232.9 & 1387.7 & 1387.7 \\
\hline 258 & & & 30.3 & 256.0 & 251.0 & & 229.7 & 198.2 & 253.3 & 955.2 & 1618.7 & 2932.8 & 4533.0 & 6472.1 & & 6472.1 \\
\hline VL-18 & & & 148.0 & 638.0 & 428.0 & 186.0 & 77.8 & 99.5 & 307.1 & 365.0 & 394.3 & 459.2 & 256.9 & 267.9 & 262.7 & 638.0 \\
\hline 229 & & & 148.0 & 638.0 & 428.0 & 186.0 & & & & & & & & & & 638.0 \\
\hline 234 (AL) & & & 8.0 & 8.0 & 7.0 & 7.3 & 6.0 & 6.5 & 5.8 & 5.7 & 6.9 & 12.6 & 5.3 & 5.7 & 5.6 & 12.6 \\
\hline 240 & & & 7.7 & 8.6 & 7.9 & 8.2 & 7.8 & 7.9 & 7.7 & 7.1 & 7.6 & 7.4 & 8.6 & 6.8 & 6.7 & 8.6 \\
\hline 248 & & & 8.7 & 11.8 & 14.0 & 15.6 & 17.8 & 20.8 & 23.8 & 29.4 & 33.6 & 45.5 & 41.0 & 44.2 & 46.8 & 46.8 \\
\hline 250 & & & 10.1 & 15.5 & 16.8 & 18.2 & 21.5 & 29.6 & 42.0 & 52.0 & 61.4 & 246.3 & 67.2 & 71.0 & 72.2 & 246.3 \\
\hline 254 & & & 16.9 & 61.1 & 63.4 & 61.7 & 71.6 & 86.3 & 139.0 & 157.8 & 220.5 & 459.2 & 256.9 & 267.9 & 262.7 & 459.2 \\
\hline 257 & & & 28.4 & 54.9 & 48.7 & 41.5 & 77.8 & 99.5 & 307.1 & 365.0 & 394.3 & & & & & 394.3 \\
\hline VL-18-Auger & & & 32.1 & 39.1 & 141.0 & 33.6 & 99.8 & 182.3 & 351.6 & 460.6 & 472.6 & & 434.6 & 414.6 & 370.5 & 472.6 \\
\hline 229 & & & & & 141.0 & & 8.8 & & & & & & & & & 141.0 \\
\hline 234 (AL) & & & 6.1 & 6.6 & 6.7 & 6.7 & 6.1 & 2.1 & 5.3 & 5.0 & 5.7 & & 5.3 & 4.4 & 4.7 & 6.7 \\
\hline 240 & & & 6.6 & 8.3 & 7.3 & 9.4 & 6.0 & 6.0 & 6.0 & 6.6 & 6.6 & & 6.4 & 6.8 & & 9.4 \\
\hline 248 & & & 14.3 & 31.0 & 25.1 & 21.4 & 20.0 & 21.1 & 20.8 & 28.3 & 35.7 & & 47.7 & 52.5 & 59.4 & 59.4 \\
\hline 250 & & & & 25.8 & 25.2 & & & 25.8 & 34.8 & 52.2 & 78.9 & & 97.1 & 102.4 & 103.3 & 103.3 \\
\hline 254 & & & 20.1 & 39.1 & 37.4 & 33.6 & 41.7 & 182.3 & 351.6 & 460.6 & 472.6 & & 434.6 & 414.6 & 370.5 & 472.6 \\
\hline 257 & & & 32.1 & 37.4 & 35.0 & & 99.8 & & & & & & & & & 99.8 \\
\hline VL-19 & & & 163.0 & 264.0 & 181.0 & 174.0 & 145.8 & 7.4 & 8.4 & 9.0 & 8.4 & 7.9 & 7.7 & 6.7 & 5.6 & 264.0 \\
\hline 230 & & & 163.0 & 264.0 & 181.0 & 174.0 & 145.8 & & & & & & & & & 264.0 \\
\hline $238(\mathrm{AL})$ & & & 7.9 & 8.4 & 7.4 & 7.2 & 6.9 & 7.4 & 8.4 & 8.5 & 8.4 & 7.9 & 7.7 & 6.7 & 5.6 & 8.5 \\
\hline 245 & & & 7.4 & 14.8 & & & & & & & & & & & & 14.8 \\
\hline
\end{tabular}


SRNL-STI-2016-00546

Revision 0

\begin{tabular}{|c|c|c|c|c|c|c|c|c|c|c|c|c|c|c|c|c|}
\hline H-3 Conc. [pCi/mL] & 2001 & 2002 & 2003 & 2004 & 2005 & 2006 & 2007 & 2008 & 2009 & 2010 & 2011 & 2012 & 2013 & 2014 & 2015 & $\begin{array}{l}\text { Max. } \\
\text { conc. }\end{array}$ \\
\hline 252 & & & 7.9 & 8.4 & 7.6 & & & & & & & & & & & 8.4 \\
\hline 255 & & & & 9.2 & 8.7 & 9.6 & 10.6 & & & & & & & & & 10.6 \\
\hline 259 & & & 10.5 & 11.5 & 10.9 & & & & & 9.0 & & & & & & 11.5 \\
\hline VL-20 & & & 12.6 & 50.2 & 27.3 & 17.0 & 17.6 & 18.3 & 10.9 & 161.7 & 11.9 & 16.7 & 14.5 & 7.0 & 7.3 & 161.7 \\
\hline 230 & & & 7.5 & & & & & & & & & & & & & 7.5 \\
\hline $240(\mathrm{AL})$ & & & & & & 6.6 & & & & & & & & & & 6.6 \\
\hline 243 & & & 9.6 & 8.8 & 8.2 & 7.5 & 7.1 & 6.5 & 5.8 & 161.7 & 5.9 & 6.0 & 6.2 & 5.8 & & 161.7 \\
\hline 258 & & & 12.6 & 50.2 & 27.3 & 17.0 & 17.6 & 18.3 & 10.9 & 8.2 & 11.9 & 16.7 & 14.5 & 7.0 & 7.3 & 50.2 \\
\hline VL-21 & & & 8.7 & 9.3 & 9.8 & 6.7 & 5.9 & 15.7 & 4.0 & 4.6 & 88.2 & 6.1 & & & 5.7 & 88.2 \\
\hline 228 & & & 5.6 & 6.8 & 6.7 & 6.7 & 5.9 & & & & & & & & & 6.8 \\
\hline 235 & & & & & & & 3.7 & & & & & & & & & 3.7 \\
\hline 239 & & & 6.2 & 7.2 & 5.4 & 5.2 & 4.3 & 4.6 & 4.0 & 4.6 & 88.2 & 6.1 & & & 5.7 & 88.2 \\
\hline 246 & & & 6.6 & 8.8 & 9.8 & & & 15.7 & & & & & & & & 15.7 \\
\hline 254 & & & 5.4 & & & & & & & & & & & & & 5.4 \\
\hline 256 & & & 8.7 & 9.3 & & & & & & & & & & & & 9.3 \\
\hline VL-22 & & & 6.6 & 6.6 & 11.2 & 72.5 & 272.9 & 391.7 & 409.6 & 376.7 & 380.5 & 356.9 & & & 404.8 & 409.6 \\
\hline $230(\mathrm{AL})$ & & & 6.6 & 6.5 & 5.7 & 5.6 & 5.2 & 5.0 & 4.3 & 4.0 & 4.1 & 4.5 & & & 48.6 & 48.6 \\
\hline 241 & & & 5.6 & 6.3 & 5.2 & 4.9 & 4.5 & 5.3 & 8.6 & 27.4 & 165.1 & 191.5 & & & 245.8 & 245.8 \\
\hline 251 & & & 5.5 & 5.9 & 6.0 & 13.8 & 55.0 & 121.7 & 263.1 & 373.1 & 380.5 & 356.9 & & & 404.8 & 404.8 \\
\hline 258 & & & 5.1 & 6.6 & 11.2 & 72.5 & 272.9 & 391.7 & 409.6 & 376.7 & 373.0 & 300.7 & & & 364.9 & 409.6 \\
\hline VL-23 & & & 10.8 & 8.3 & 6.2 & 6.4 & 5.2 & 5.6 & 5.2 & 5.5 & 4.7 & 5.2 & & 11.8 & 121.2 & 121.2 \\
\hline $230(\mathrm{AL})$ & & & & 6.8 & 6.2 & 6.3 & 5.2 & 5.6 & 4.6 & 4.7 & 4.7 & 5.2 & & 11.8 & 121.2 & 121.2 \\
\hline 241 & & & 6.2 & 8.3 & 6.1 & 6.4 & & 5.6 & 5.2 & 5.5 & & & & & & 8.3 \\
\hline 247 & & & 10.8 & 6.3 & & & & & & & & & & & & 10.8 \\
\hline VL-6-South_Center & 11.4 & 11.9 & 18.5 & 36.2 & 32.0 & 42.6 & 39.4 & 44.8 & 53.8 & & 41.5 & 49.4 & 502.1 & 342.8 & 389.0 & 502.1 \\
\hline 219 & 11.4 & & & 10.7 & 9.3 & 7.7 & & & & & & & & & & 11.4 \\
\hline $233(\mathrm{AL})$ & & 11.9 & 10.8 & 8.7 & 26.2 & 36.3 & 39.4 & & 34.6 & & 41.5 & 49.4 & 502.1 & & 389.0 & 502.1 \\
\hline 241 & & 5.4 & 18.5 & 36.2 & 32.0 & 42.6 & 29.7 & 44.8 & 53.8 & & & & 432.9 & 342.8 & 134.6 & 432.9 \\
\hline
\end{tabular}




\begin{tabular}{|c|c|c|c|c|c|c|c|c|c|c|c|c|c|c|c|c|}
\hline H-3 Conc. [pCi/mL] & 2001 & 2002 & 2003 & 2004 & 2005 & 2006 & 2007 & 2008 & 2009 & 2010 & 2011 & 2012 & 2013 & 2014 & 2015 & $\begin{array}{l}\text { Max. } \\
\text { conc. }\end{array}$ \\
\hline VL-7-SE_Corner & 10.8 & 10.5 & 5.1 & 14.7 & 9.2 & 9.1 & 9.1 & 9.2 & 5.8 & 5.4 & 5.3 & 5.7 & 6.5 & 9.3 & 9.2 & 14.7 \\
\hline 214 & & & & & & 7.4 & & & & & & & & & & 7.4 \\
\hline 219 & 10.8 & 10.5 & & & & & & & & & & & & & & 10.8 \\
\hline 233 & & 9.5 & & & & & & & & & & & & & & 9.5 \\
\hline $235.7(\mathrm{AL})$ & & & & & & & & & 5.2 & 5.1 & 5.0 & 5.1 & 5.5 & 9.3 & 9.2 & 9.3 \\
\hline 242 & & 5.6 & 5.1 & 14.7 & 9.2 & 9.1 & 9.1 & 9.2 & 5.8 & 5.4 & 5.3 & 5.7 & 6.5 & 5.9 & 5.4 & 14.7 \\
\hline VL-8-East_Center & 8.3 & 9.3 & 140.0 & 220.0 & 148.0 & 111.0 & 66.5 & 95.5 & 54.3 & 69.7 & 108.4 & 138.6 & 149.8 & 101.6 & 63.0 & 220.0 \\
\hline 220 & 5.6 & & & 6.7 & & & & & & & & & & & & 6.7 \\
\hline 233 & 7.0 & & & & & & & & & & & & & & & 7.0 \\
\hline $234.9(\mathrm{AL})$ & & & & & & & & & 9.8 & 16.4 & 29.4 & 41.1 & 48.9 & 64.0 & 63.0 & 64.0 \\
\hline 241 & 8.3 & 9.3 & 140.0 & 220.0 & 148.0 & 111.0 & 66.5 & 95.5 & 54.3 & 69.7 & 108.4 & 138.6 & 149.8 & 101.6 & 45.3 & 220.0 \\
\hline VL-9-NE & 5.1 & 6.5 & 6.0 & 6.7 & 4.3 & 4.4 & 4.2 & & & & 4.1 & 3.8 & 4.4 & & & 6.7 \\
\hline 213 & & & & & & 4.4 & 4.2 & & & & & & & & & 4.4 \\
\hline 219 & 5.0 & 6.5 & & & & & & & & & & & & & & 6.5 \\
\hline 233 & & 6.3 & 6.0 & 6.7 & 4.3 & 4.4 & & & & & & & & & & 6.7 \\
\hline 241 & 5.1 & & & & & & & & & & 4.1 & 3.8 & 4.4 & & & 5.1 \\
\hline Max. conc. & 11.4 & 11.9 & 163.0 & 638.0 & 1003.0 & 3040.0 & 3151.8 & 2051.7 & 1831.0 & 2447.7 & 2445.5 & 2932.8 & 4533.0 & 6472.1 & 1434.5 & 6472.1 \\
\hline
\end{tabular}


The average concentration of tritium expected in the waste zone can be estimated from the WITS inventory $(2.21 \mathrm{Ci})$ and estimated material properties and saturation state. Table 3-2 summarizes a calculation assuming boxed waste (Phifer and Wilhite 2001) and waste zone soil saturation based on three assumptions: gravity equilibrium (no infiltration), 100\% saturation, and field measurements (Phifer et al. 2006). Initial H-3 concentrations range from 742 to $1348 \mathrm{pCi} / \mathrm{mL}$ depending on the saturation assumption. Although dispersion and decay will attenuate tritium as it migrates from the waste zone into the shallow vadose zone, this calculation suggests that concentrations averaging several hundred $\mathrm{pCi} / \mathrm{mL}$ can be expected from shallow lysimeters.

Because the VZMS tritium data vary widely, spanning approximately three orders of magnitude, average concentrations observed in the field just beneath the ET1 waste zone are not obvious from simple visual inspection of the spatial-temporal data. However, statistical techniques can be applied to subsets of the overall database to estimate representative concentrations (e.g. mean, median) and uncertainty ranges. In the statistical analysis that follows, concentrations acquired from lysimeters positioned around the perimeter of the disposal unit are assumed to be representative of the entire disposal area.

Going forward, two data subsets are considered to characterize tritium release to the shallow vadose zone. The first data population is composed of the peak H-3 concentration observed at any point in time for the shallow-most lysimeter at each plan-view location. For brevity these concentrations are identified using labels such as "Max_high" or "MaxAtHighElev". The intent of this dataset is to include the peak concentrations leaving the ET1 waste zone and entering undisturbed native soil regardless of timing, recognizing that varying disposal times and container integrity likely resulted in varying plume arrival times at the various lysimeters. The second data population is a snapshot of $\mathrm{H}-3$ concentrations observed in the shallow-most lysimeters in FY2015. The intent of this dataset is to consider near current conditions directly beneath the waste zone. This dataset is identified with abbreviated labels such as "2015_high" and "2015AtHighElev".

A random process is described most completely through a detailed statistical distribution relating values to probability of occurrence, for example, a normal distribution $\mathbb{N}$ with mean $\mu$ and variance $\sigma^{2}, \mathbb{N}\left(\mu, \sigma^{2}\right)$. Figure 3-4 illustrates normal probability plots for (a) untransformed concentration and (b) the base 10 logarithm of concentration, based on the "Max_high" dataset. The observed degrees of fit indicate that VZMS concentrations are much better approximated by a log-normal distribution than a normal distribution.

Key characteristics of a distribution can be identified through various statistical measures, or statistics. Common statistics are the mean and median which define a representative central value, and the variance (or equivalently standard deviation) which measures the spread of the population. The statistical mean is the arithmetic average of the random variable or population. The median is the value for which half of the population values are below and the other half are above (50\% cumulative probability).

The means of the "Max_high" and "2015_high" data are $m=(1 / N) \sum_{i=1}^{N} c_{i}=536$ and $247 \mathrm{pCi} / \mathrm{mL}$, respectively, where $N$ is the number of samples and $c$ is concentration. These simple arithmetic averages do not account for data location or potential clustering. The spatial average of the interpolated "2015_high" data in Figure 3-3 is $228 \mathrm{pCi} / \mathrm{mL}$. Thus accounting for data location slightly lowers the estimated mean $(-8 \%)$. Because this difference is small, further statistical analyses ignore data locations and give equal weight to each lysimeter location. 
Table 3-2. Estimated initial concentration of tritium in the ET1 waste zone.

\begin{tabular}{|c|c|c|c|}
\hline Parameter & Value & Units & Comments \\
\hline \multirow[t]{2}{*}{ Trench length, $\mathrm{L}$} & 650 & $\mathrm{ft}$ & \\
\hline & 198.1 & $\mathrm{~m}$ & \\
\hline \multirow[t]{2}{*}{ Trench width, W } & 160 & $\mathrm{ft}$ & \\
\hline & 48.8 & $\mathrm{~m}$ & \\
\hline \multirow[t]{2}{*}{ Cover thickness, D } & 4 & $\mathrm{ft}$ & \\
\hline & 1.22 & $\mathrm{~m}$ & \\
\hline \multirow[t]{2}{*}{ Waste zone thickness, D } & 17.3 & $\mathrm{ft}$ & B-25 boxes, stacked four high \\
\hline & 5.27 & $\mathrm{~m}$ & \\
\hline \multirow[t]{2}{*}{ Equiv. soil thick. in waste layer, D } & 2.2 & $\mathrm{ft}$ & Based on WSRC-RP-2001-00613 \\
\hline & 0.67 & $\mathrm{~m}$ & \\
\hline Fraction of waste zone thickness & $13 \%$ & & \\
\hline \multirow[t]{2}{*}{ Vadose zone thickness, D } & 35 & $\mathrm{ft}$ & \\
\hline & 10.67 & $\mathrm{~m}$ & \\
\hline Waste volume, V & 6479 & $m^{3}$ & \\
\hline Porosity, $n$ & 0.46 & $\mathrm{~cm}^{3}$ void $/ \mathrm{cm}^{3}$ & Operational soil cover prior to compaction \\
\hline $\mathrm{H}-3$ inventory, I & 2.21 & $\mathrm{Ci}$ & As-disposed-of through 9/30/2015 \\
\hline Gravity equilbrium: & & & Expected average concentration - high estimate \\
\hline \multirow[t]{2}{*}{ Mean waste height above WT } & 13.30 & $\mathrm{~m}$ & Vadose zone + half of waste zone thickness \\
\hline & 1330 & $\mathrm{~cm}$ & Use to estimate saturation \\
\hline Saturation, S & 0.73 & $\mathrm{~cm}^{3}$ water $/ \mathrm{cm}^{3}$ void & Manual lookup: Table 5-21, WSRC-STI-2006-00198 \\
\hline Water content, $\theta$ & 0.33 & $\mathrm{~cm}^{3}$ water $/ \mathrm{cm}^{3}$ & \\
\hline Water volume, $V_{w}$ & 2161 & $m^{3}$ & $2160711 \mathrm{~L}$ \\
\hline \multirow[t]{2}{*}{$\mathrm{H}-3$ concentration, $\mathrm{C}$} & 1022811 & $\mathrm{pCi} / \mathrm{L}$ & \\
\hline & 1023 & $\mathrm{pCi} / \mathrm{mL}$ & \\
\hline Full saturation: & & & Expected average concentration - low estimate \\
\hline Saturation, S & 1.00 & $\mathrm{~cm}^{3}$ water $/ \mathrm{cm}^{3}$ void & \\
\hline Water content, $\theta$ & 0.46 & $\mathrm{~cm}^{3}$ water $/ \mathrm{cm}^{3}$ & \\
\hline Water volume, $\mathrm{V}_{\mathrm{w}}$ & 2980 & $m^{3}$ & $2980291 \mathrm{~L}$ \\
\hline \multirow[t]{2}{*}{$\mathrm{H}-3$ concentration, $\mathrm{C}$} & 741538 & $\mathrm{pCi} / \mathrm{L}$ & \\
\hline & 742 & $\mathrm{pCi} / \mathrm{mL}$ & \\
\hline Field observations: & & & Expected average concentration - field observations \\
\hline Saturation, S & 0.55 & $\mathrm{~cm}^{3}$ water $/ \mathrm{cm}^{3}$ void & Average of Sec. 5.8, WSRC-STI-2006-00198 range \\
\hline Water content, $\theta$ & 0.25 & $\mathrm{~cm}^{3}$ water $/ \mathrm{cm}^{3}$ & \\
\hline Water volume, $V_{w}$ & 1639 & $m^{3}$ & $1639160 \mathrm{~L}$ \\
\hline \multirow[t]{2}{*}{$\mathrm{H}-3$ concentration, $\mathrm{C}$} & 1348251 & $\mathrm{pCi} / \mathrm{L}$ & \\
\hline & 1348 & $\mathrm{pCi} / \mathrm{mL}$ & \\
\hline
\end{tabular}


The standard deviations of the "Max_high" and "2015_high" sample means (standard error) are $s_{m}=$ $s / \sqrt{N}=316$ and $110 \mathrm{pCi} / \mathrm{mL}$, where $s$ is the sample standard deviation:

$$
\sqrt{\frac{1}{N-1} \sum_{i=1}^{N}\left(c_{i}-m\right)^{2}}
$$

Unless a set of samples exhibits a normal distribution, the distribution of the sample mean only approaches a normal distribution in the limit $N \rightarrow \infty$ (Central Limit Theorem; Box et al. 1978). The sample counts are $N=22$ and 17 for the "Max_high" and "2015_high" datasets, respectively. Because the VZMS data exhibit a highly-skewed, approximately log-normal, population distribution (Figure 3-4), a substantially larger sample count $N$ is almost certainly required to achieve an approximately normally distributed sample mean $m$. This expectation is supported by a calculation of the $95 \%$ confidence interval for the "Max_high" sample mean, naïvely assuming a normal distribution: $m-1.96 s_{m}<\mu<m+$ $1.96 s_{m}$ where $\mu$ is the true mean of the population. The result is $-83<\mu<1156 \mathrm{pCi} / \mathrm{mL}$. The negative concentration defining the lower end of the interval is nonsensical and clear indication of non-normality. Thus uncertainty in the "Max_high" and "2015_high" sample means can only be crudely estimated assuming normality (e.g. $\pm 1.96 s_{m} \approx \pm 2 s_{m}$ or "2-sigma” uncertainty range).

A statistical bootstrapping technique (Efron, 1982) can be used to estimate the distribution of VZMS sample means, from which more accurate confidence intervals can be computed. In the bootstrap approach, the sample data are used as a surrogate for the true underlying population. Each bootstrapping realization is generated by randomly re-sampling the actual set of sample results (with replacement) to produce a new sample of the same size (e.g. a set of 15 samples would be randomly sampled 15 times for each realization). A sample mean is computed for each of many realizations. The distribution of realization sample means approximates the true distribution. Figure 3-5 summarizes bootstrapping results for the "Max_high" and "2015_high" datasets. Both sample mean distributions are skewed, but far less than the underlying sample data. The "Max_high" distribution displays several peaks, which apparently result from a small sample size $(N=22)$ and sample variability over three orders of magnitude. The "2015_high" distribution is much smoother, and probably a better approximation of its true population distribution. The median (50\% cumulative probability) and 95\% confidence interval results (2.5\% and 97.5\% cumulative probability) based on bootstrapping are $80<510<1156 \mathrm{pCi} / \mathrm{mL}$ for the "Max_high" data and $65<237<485 \mathrm{pCi} / \mathrm{mL}$ for the "2015_high" concentrations as shown in Table 3-3 (a).

Because the "Max_high" bootstrapping distribution exhibits clear artifacts of small sample size, the confidence interval for the "Max_high" sample mean is also estimated assuming the sample data are lognormally distributed, based on Figure 3-4(b). The mean and standard deviation of $\log 10(c)$ are taken as the sample mean and sample standard deviation. The resulting median and 95\% confidence intervals are $84<315<1914 \mathrm{pCi} / \mathrm{mL}$, which is similar to the bootstrapping result (Table 3-3 (b)).

An alternative approach for analyzing VZMS sample data is to generate samples of $\log 10(c)$, compute the sample mean $(\mathrm{m})$ and standard deviation $\left(s_{m}\right)$ of $\log 10(\mathrm{c})$ for each realization, and estimate the sample mean of $c$ by assuming a log-normal distribution and using the back transformation (Appendix D in Flach 2002):

$$
10^{m+s_{m}^{2} \ln (10) / 2}
$$

This approach can be applied to bootstrapping samples or samples drawn from an assumed log-normal distribution following Table 3-3 (a) and (b), respectively. The results of the alternative approach applied 
to the "Max_high" data set are summarized in Table 3-3 (c) and (d). The results from the four analyses of "Max_high" are similar.

While Figure 3-4 indicates that a log-normal distribution better represents the sample data distribution than a normal distribution, the data distribution can be refined by optimizing a Box-Cox transformation of the sample set (Box et al. 1978, Section 7.9). For the "Max_high" data set the optimal power $\lambda$ is -0.2 , which is close to a log-normal distribution $(\lambda=0)$ and supports the log-normal approximations discussed above.

Qualitatively, the average sample concentrations appear to be consistent with the range of $\mathrm{H}-3$ average concentrations estimated to initially reside in the ET1 waste zone (Table 3-2). However, decay and dispersion will attenuate the waste zone concentration as the plume migrates toward shallow lysimeter locations, precluding direct comparison of Table 3-2 and Table 3-3. To remedy this disconnect, subsurface flow and solute transport modeling is used in the next section to estimate $\mathrm{H}-3$ concentrations in the undisturbed vadose zone beneath ET1.

Also of interest are tritium concentrations in the deep vadose zone from ET1 Action Level lysimeters, where lower concentrations are expected due to further decay and dispersion. Table 5-2 of Hiergesell et al. (2016) presents these data for FY2015. Statistical results for this dataset are presented in Figure 3-6 and Table 3-3. 


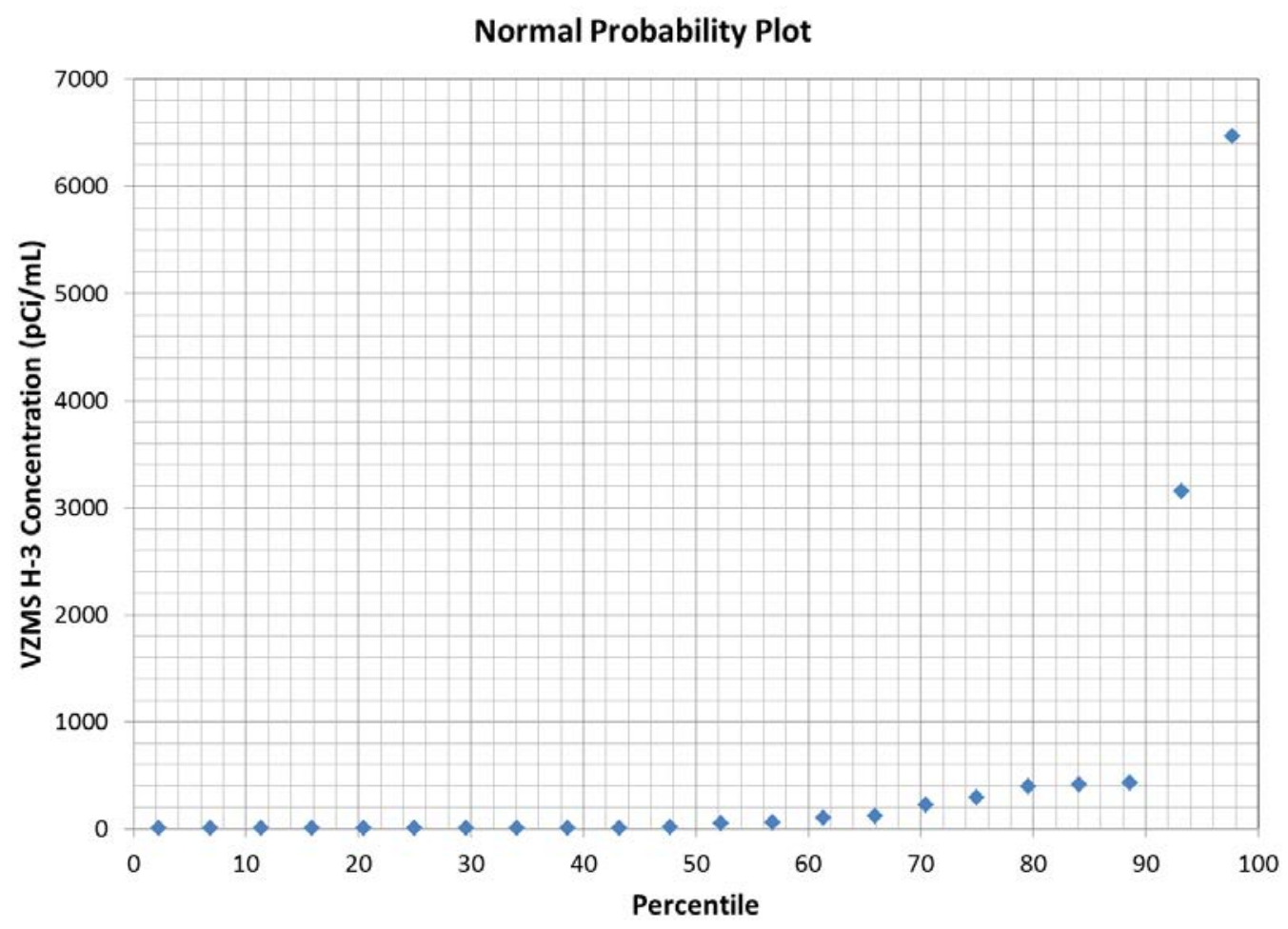

(a)

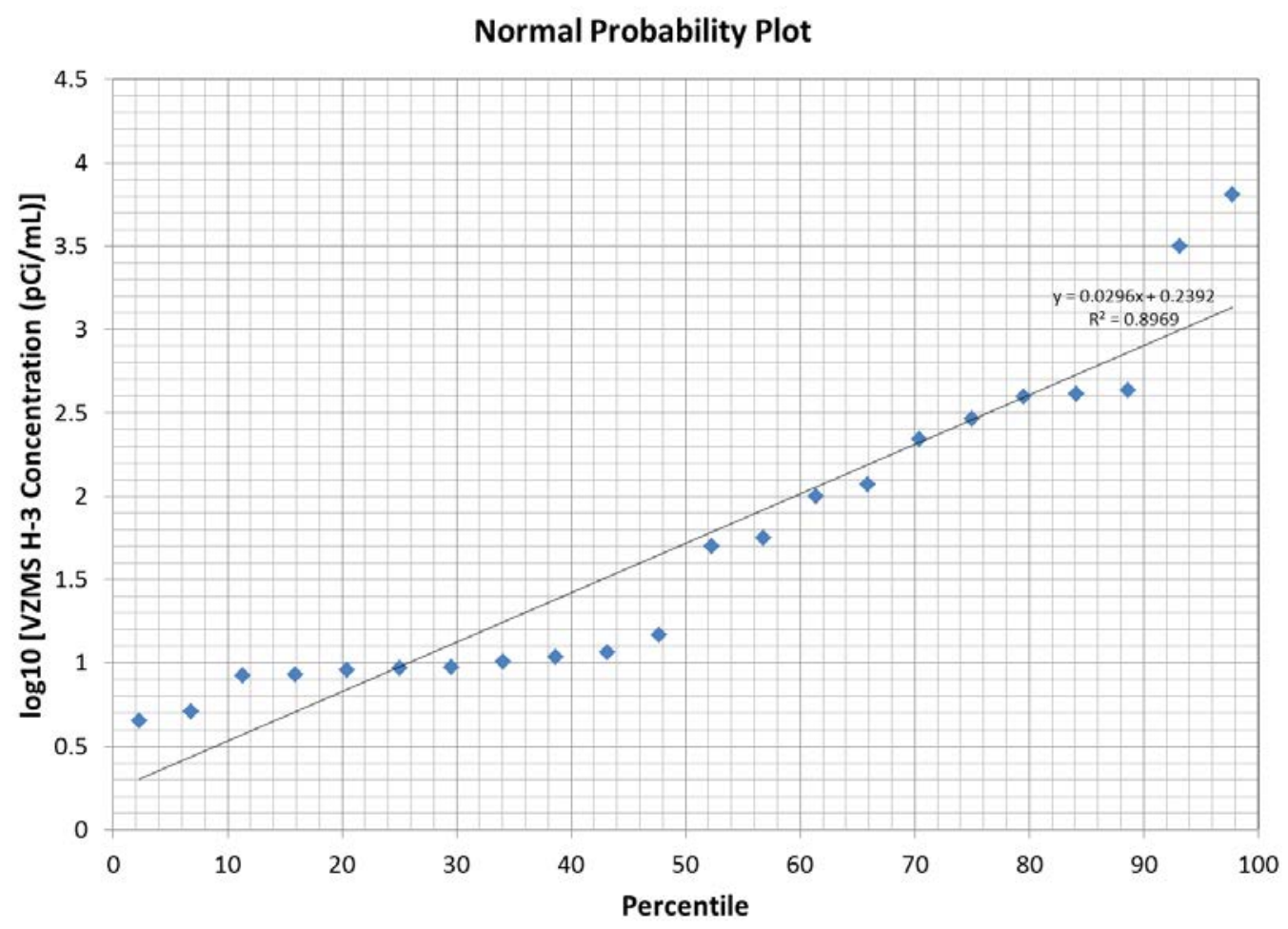

(b)

Figure 3-4. Normal probability plots for concentration and log10[concentration] using “Max_high” dataset. 


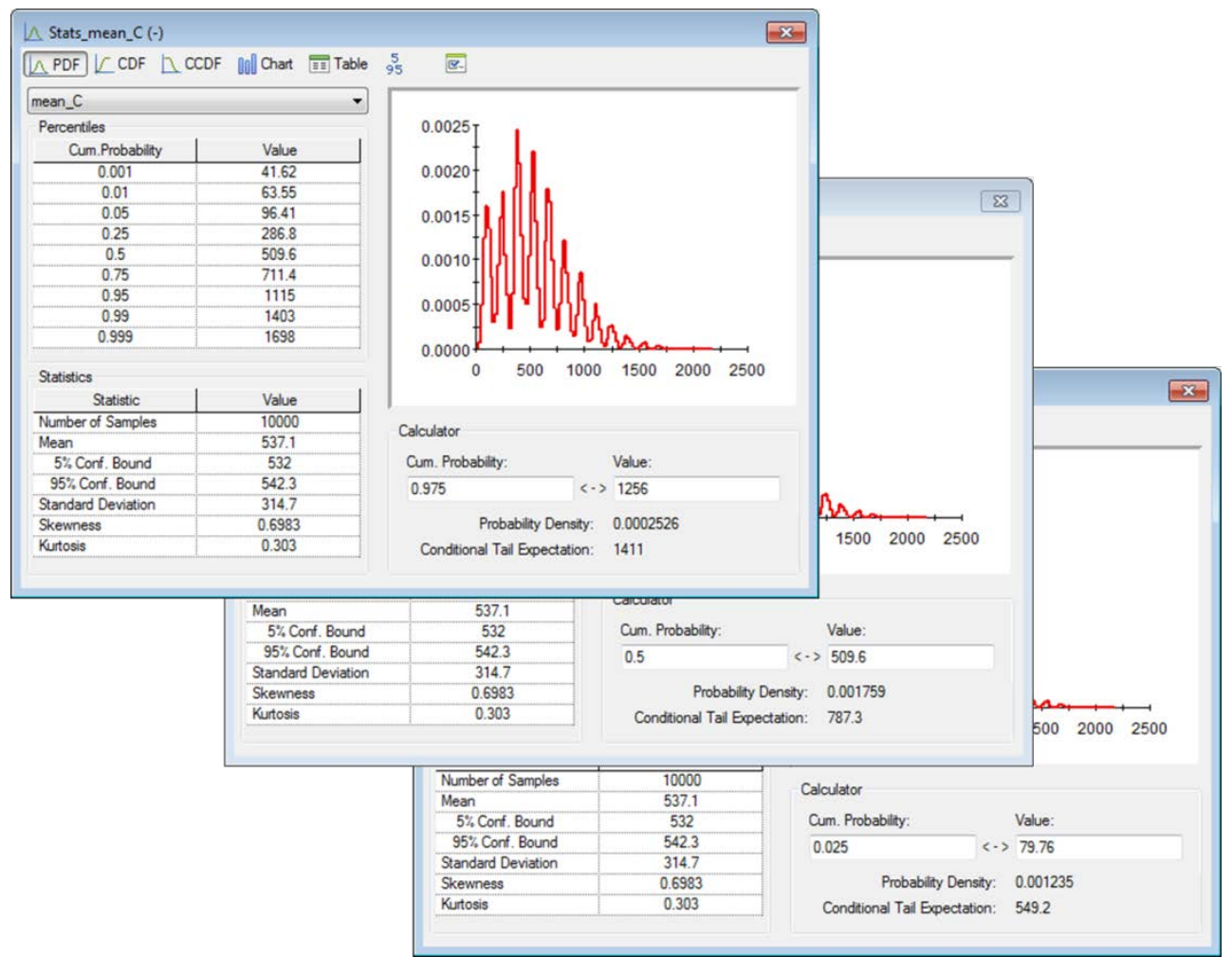

(a)

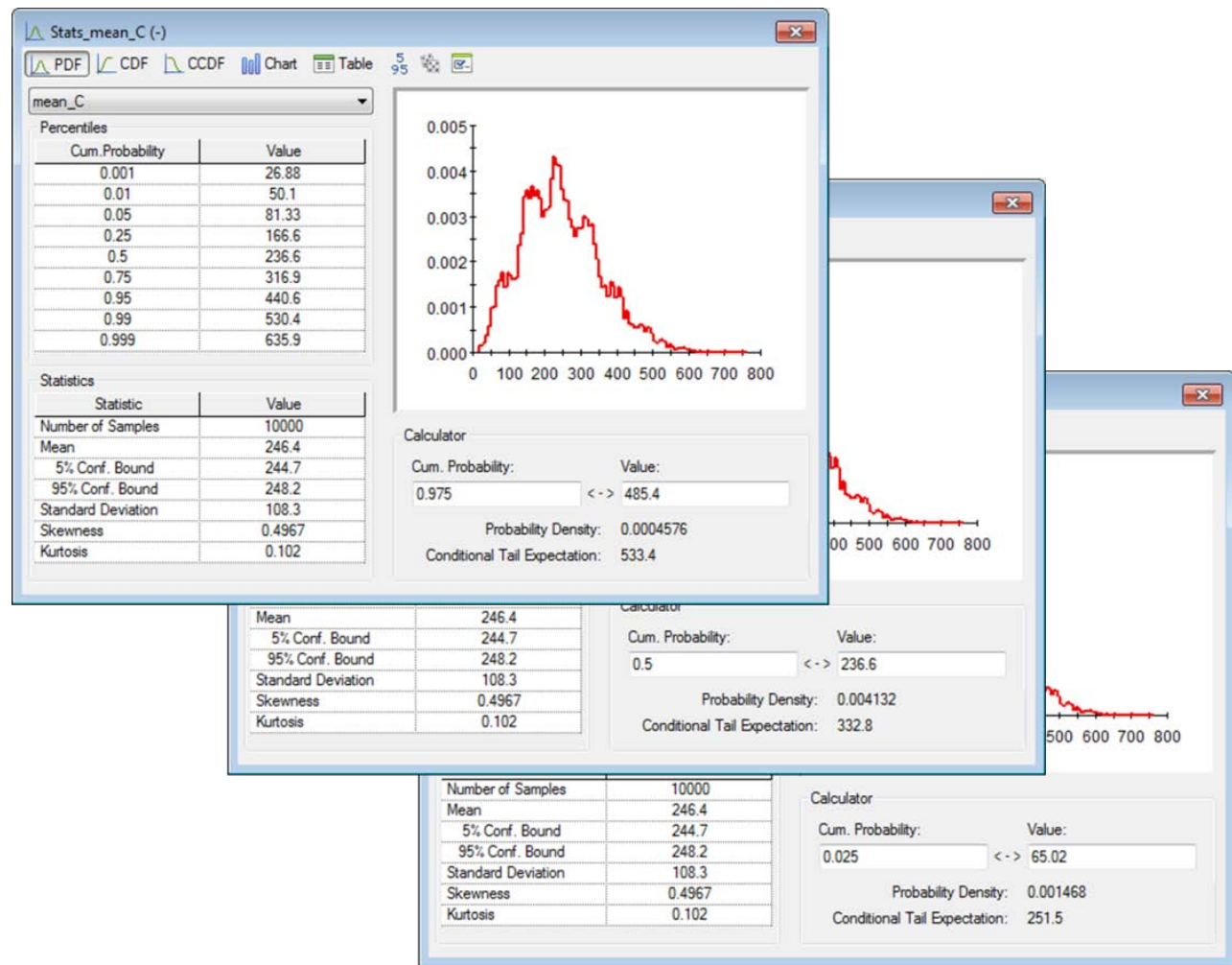

(b)

Figure 3-5. Bootstrapping results for the (a) “Max_high” and (b) “2015_high” datasets. 
Table 3-3. Summary of statistical analysis of VZMS data.

\begin{tabular}{|c|c|c|c|}
\hline Dataset: & "Max_high" & "2015_high" & 2015 Action Level \\
\hline \multicolumn{4}{|l|}{ Standard sample statistics } \\
\hline Sample count & 22 & 17 & 16 \\
\hline Sample mean & 536 & 247 & 121 \\
\hline Spatial average based on kriging interpolation & - & 228 & - \\
\hline Standard deviation of the mean (Standard error) & 316 & 110 & 68 \\
\hline \multicolumn{4}{|c|}{ Statistics on the Sample Mean } \\
\hline \multicolumn{4}{|c|}{ (a) Bootstrapping technique w/ Sampling concentration } \\
\hline $2.5 \%$ cumulative probability, Lower limit of $95 \% \mathrm{Cl}^{\star}$ & 80 & 65 & 22 \\
\hline $25 \%$ cumulative probability & 287 & 167 & 72 \\
\hline $50 \%$ cumulative probability, Median & 510 & 237 & 112 \\
\hline $75 \%$ cumulative probability & 711 & 317 & 161 \\
\hline $97.5 \%$ cumulative probability, Upper limit of $95 \% \mathrm{Cl}$ & 1156 & 485 & 267 \\
\hline \multicolumn{4}{|c|}{ (b) Log-normal distribution assumption w/ Sampling concentration } \\
\hline $2.5 \%$ cumulative probability, Lower limit of $95 \% \mathrm{Cl}$ & 84 & - & - \\
\hline $25 \%$ cumulative probability & 193 & - & - \\
\hline $50 \%$ cumulative probability, Median & 315 & - & - \\
\hline $75 \%$ cumulative probability & 541 & - & - \\
\hline $97.5 \%$ cumulative probability, Upper limit of $95 \% \mathrm{Cl}$ & 1914 & - & - \\
\hline \multicolumn{4}{|c|}{ (c) Bootstrapping technique w/ Sampling log10(concentration) } \\
\hline $2.5 \%$ cumulative probability, Lower limit of $95 \% \mathrm{Cl}$ & 75 & - & - \\
\hline $25 \%$ cumulative probability & 238 & - & - \\
\hline $50 \%$ cumulative probability, Median & 453 & - & - \\
\hline $75 \%$ cumulative probability & 862 & - & - \\
\hline $97.5 \%$ cumulative probability, Upper limit of $95 \% \mathrm{Cl}$ & 2873 & - & - \\
\hline \multicolumn{4}{|c|}{ (d) Log-normal distribution assumption w/ Sampling log10(concentration) } \\
\hline $2.5 \%$ cumulative probability, Lower limit of $95 \% \mathrm{Cl}$ & 109 & - & - \\
\hline $25 \%$ cumulative probability & 279 & - & - \\
\hline $50 \%$ cumulative probability, Median & 480 & - & - \\
\hline $75 \%$ cumulative probability & 858 & - & - \\
\hline $97.5 \%$ cumulative probability, Upper limit of $95 \% \mathrm{Cl}$ & 3081 & - & - \\
\hline
\end{tabular}

${ }^{*} \mathrm{Cl}=$ Confidence Interval 
SRNL-STI-2016-00546

Revision 0

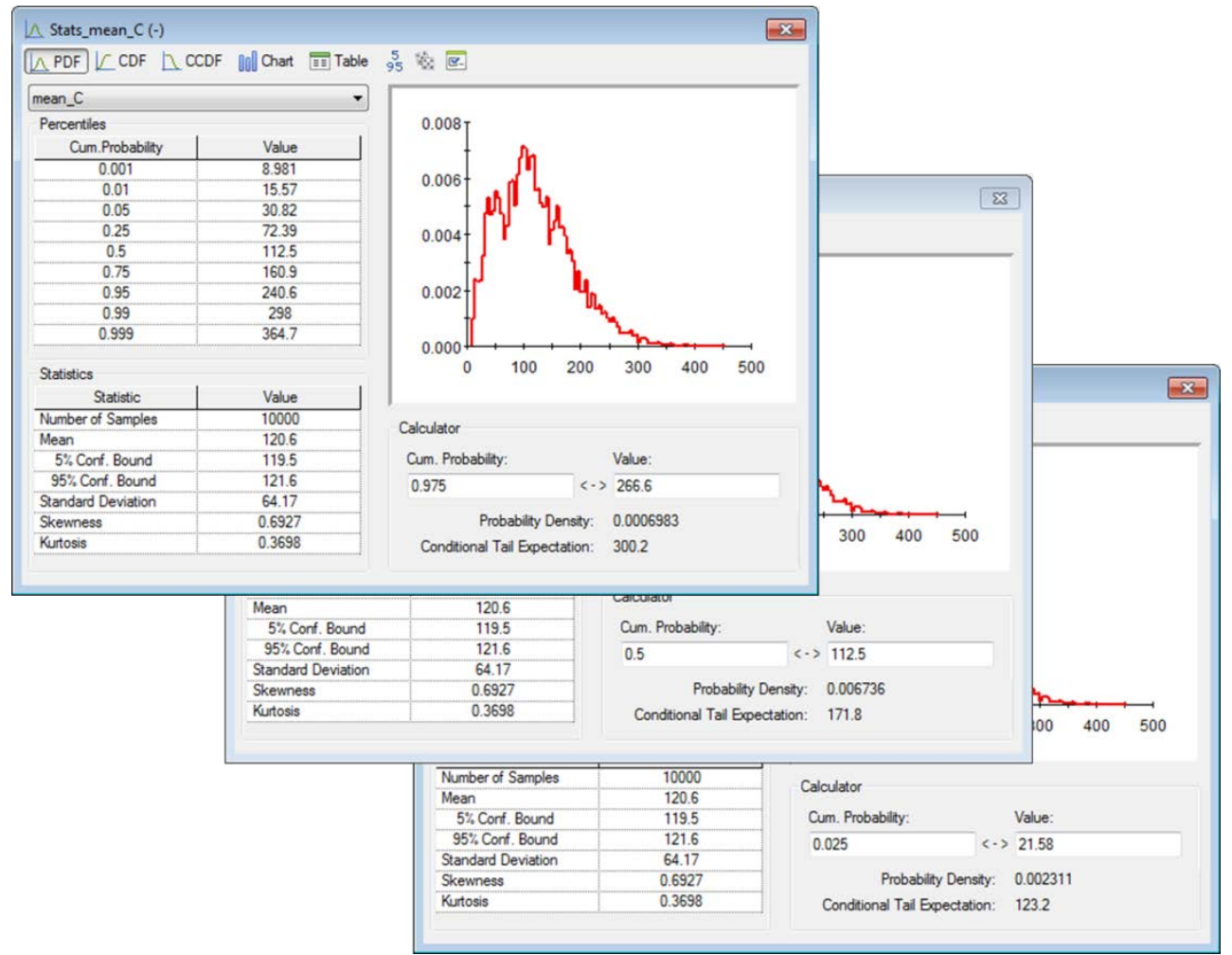

Figure 3-6. Bootstrapping results for FY2015 ET1 Action Level lysimeter dataset. 


\subsection{Radionuclide transport simulations}

Performance Assessment simulations of radionuclide transport from disposal units to the 100-meter perimeter are composed of two-dimensional vadose zone flow and transport simulations and a threedimensional aquifer transport simulation using a flow field extracted from the GSA/PORFLOW model (Flach 2004).

\subsection{Vadose zone model simulations}

A vadose zone flow and solute transport model for Engineered Trenches was developed for the 2008 E-Area Performance Assessment (WSRC 2008). Because model development occurred while disposal operations were underway for ET1 and predated ET2 operation, hypothetical conditions were assumed regarding waste disposal timing and distribution. Specifically, the 2008 PA assumed: (a) uniform waste disposal within the entire excavated volume (including side slopes), (b) the disposal unit was filled to capacity in a single instant, and (c) soil backfilled the entire excavated volume (no consideration of waste containers).

The as-disposed-of conditions of ET1 are: (a) non-uniform waste distribution excluding use of side-slope volume, (b) disposal operations occurring over several years, and (c) most waste disposals taking the form of B-25 and similar containers with low-density contents. To better reflect as-disposed-of condition (a), the 2008 PA vadose zone model was modified in this study by confining waste inventory to the full-depth portion of the trench excavation. Secondly, the model grid was refined to reduce numerical dispersion and enable more accurate monitoring of simulated concentrations at lysimeter elevations, as shown in Figure 4-1. This initial modification to the 2008 PA model is labeled Case01.

Figure 4-2 illustrates simulated concentration for Case 01 at 1.0 year. The initial waste zone concentration is only $123 \mathrm{pCi} / \mathrm{mL}$ compared to much higher values in Table 3-3, because the waste zone is assumed to be completely filled with backfilled soil. Thus downstream plume concentrations cannot exceed 123 $\mathrm{pCi} / \mathrm{mL}$.

Figure 4-3 plots simulated H-3 concentrations at Edge and Center lysimeters at four depths: Upper, Middle, Lower and Water table elevations. VZMS data statistics from Table 3-3 are also shown for comparison, and those individual "Max_high" concentrations greater than $10 \mathrm{pCi} / \mathrm{mL}$. The elapsed times for the sample data are the difference between the sample acquisition date and the estimated date of burial of nearby waste. The Upper_Edge and Upper_Center locations correspond most closely to the selected shallow VZMS data. Several VZMS data peaks occur after the time window predicted by the model. This discrepancy may be due to delays in waste release due to waste containment in B-25 boxes.

Although the magnitudes of the simulated Upper_Edge and Upper_Center concentrations are within the 95\% confidence intervals for VZMS data, they fall close to the lower bound of the interval. This observation suggests a systemic model bias. The most likely cause of model bias in Case01 is the assumption of $100 \%$ backfill soil in the waste zone, which leads to a low initial concentration as discussed earlier. Table 3-2 indicates that a substantial increase in concentration can be realized by accounting for the low-density of uncompacted boxed waste. Figure 4-3through Figure 4-8 illustrate the results from five additional modeling cases that account for the B-25 box disposals in ET1. 


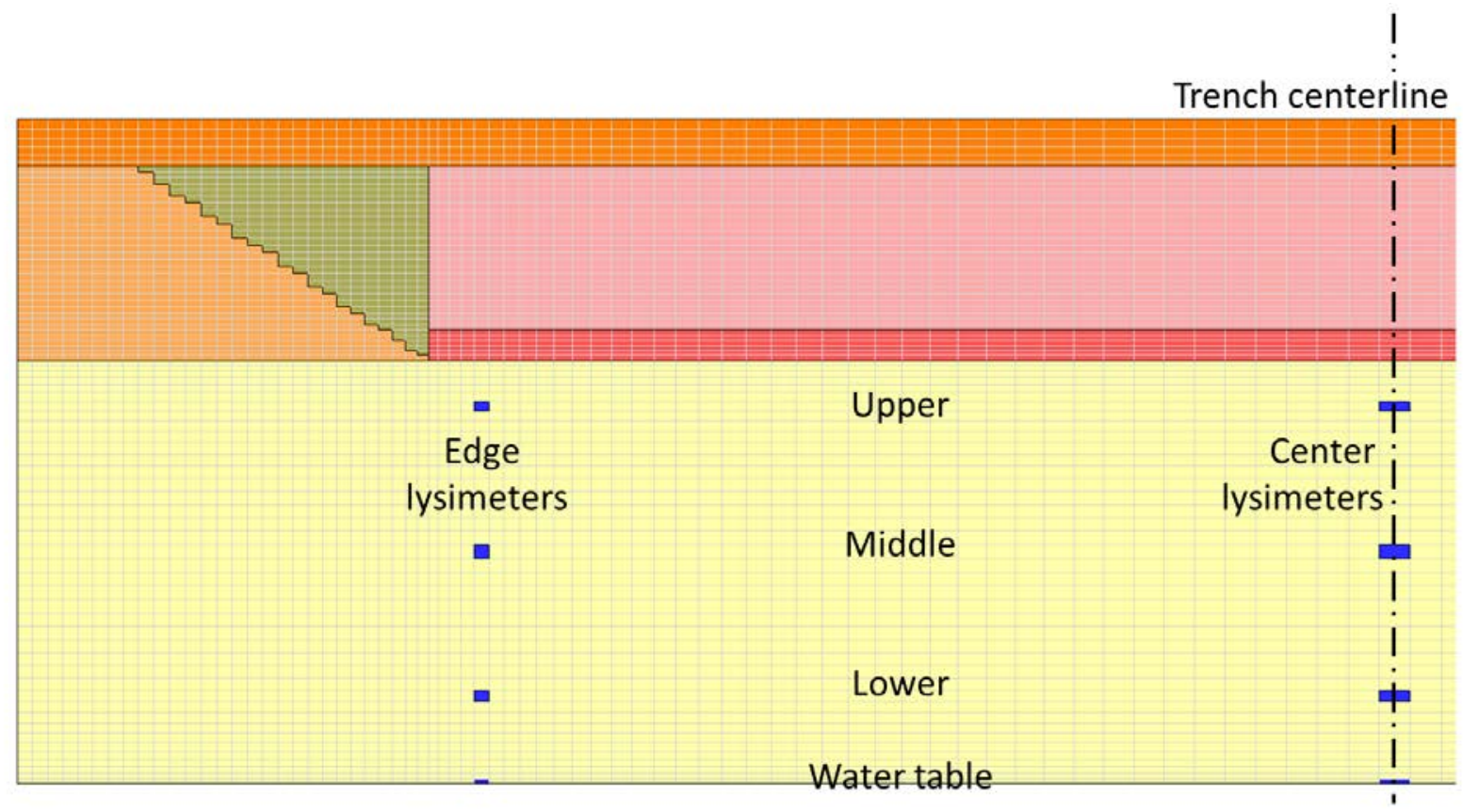

Figure 4-1. Refined vadose zone grid and added edge and center lysimeter observation points.

C_1.00

$\mathrm{pCi} / \mathrm{mL}$

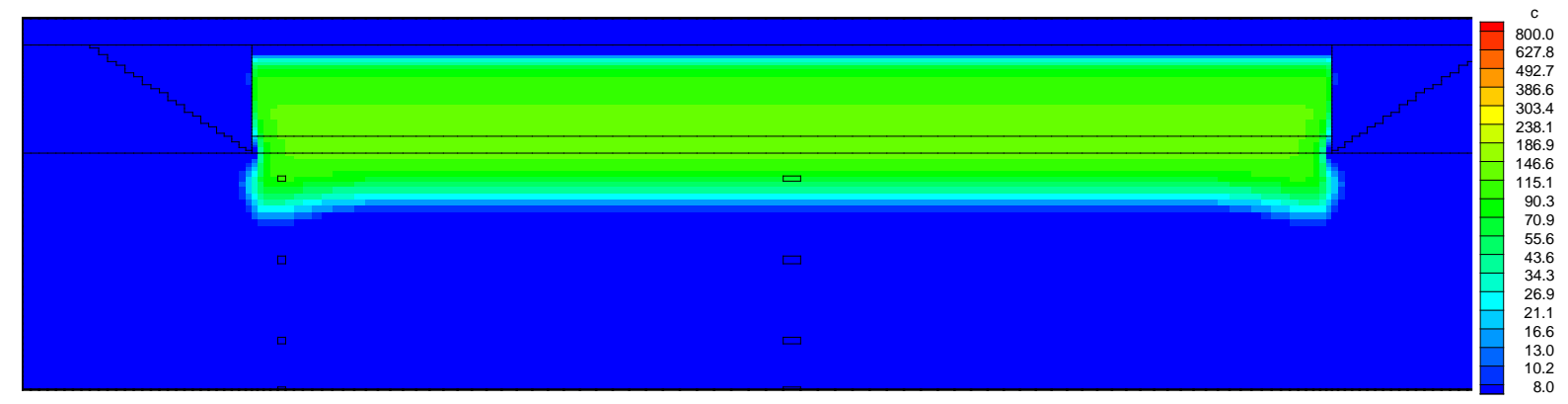

Figure 4-2. Simulated $\mathrm{H}-3$ plume for Case01 at an elapsed time of $1.0 \mathrm{yr}$. 


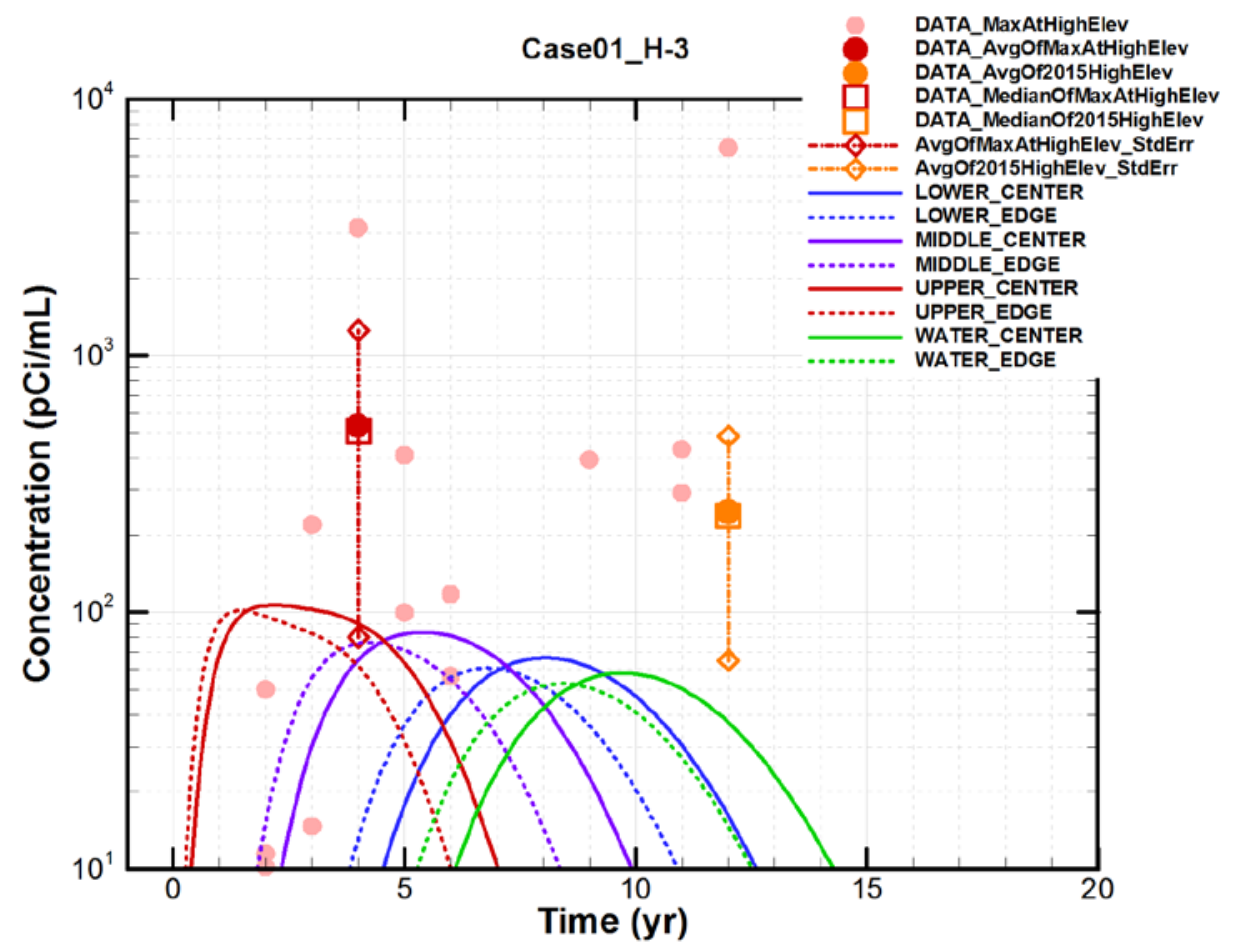

Figure 4-3. Simulated vadose zone concentrations for modeling Case01 -- PA + grid refinement + no side slope usage.

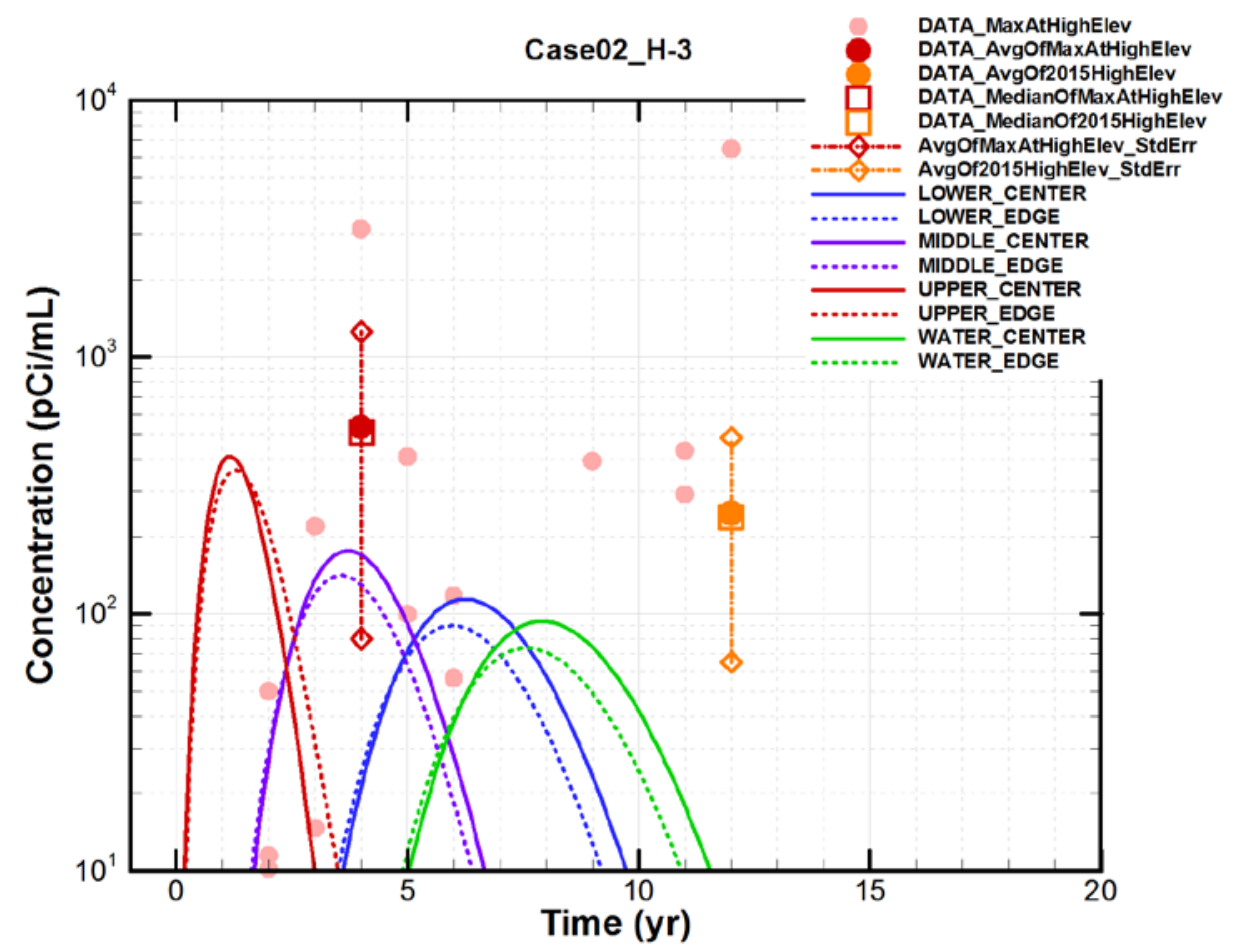

Figure 4-4. Simulated vadose zone concentrations for modeling Case02 -- B-25 boxes + no dispersion. 


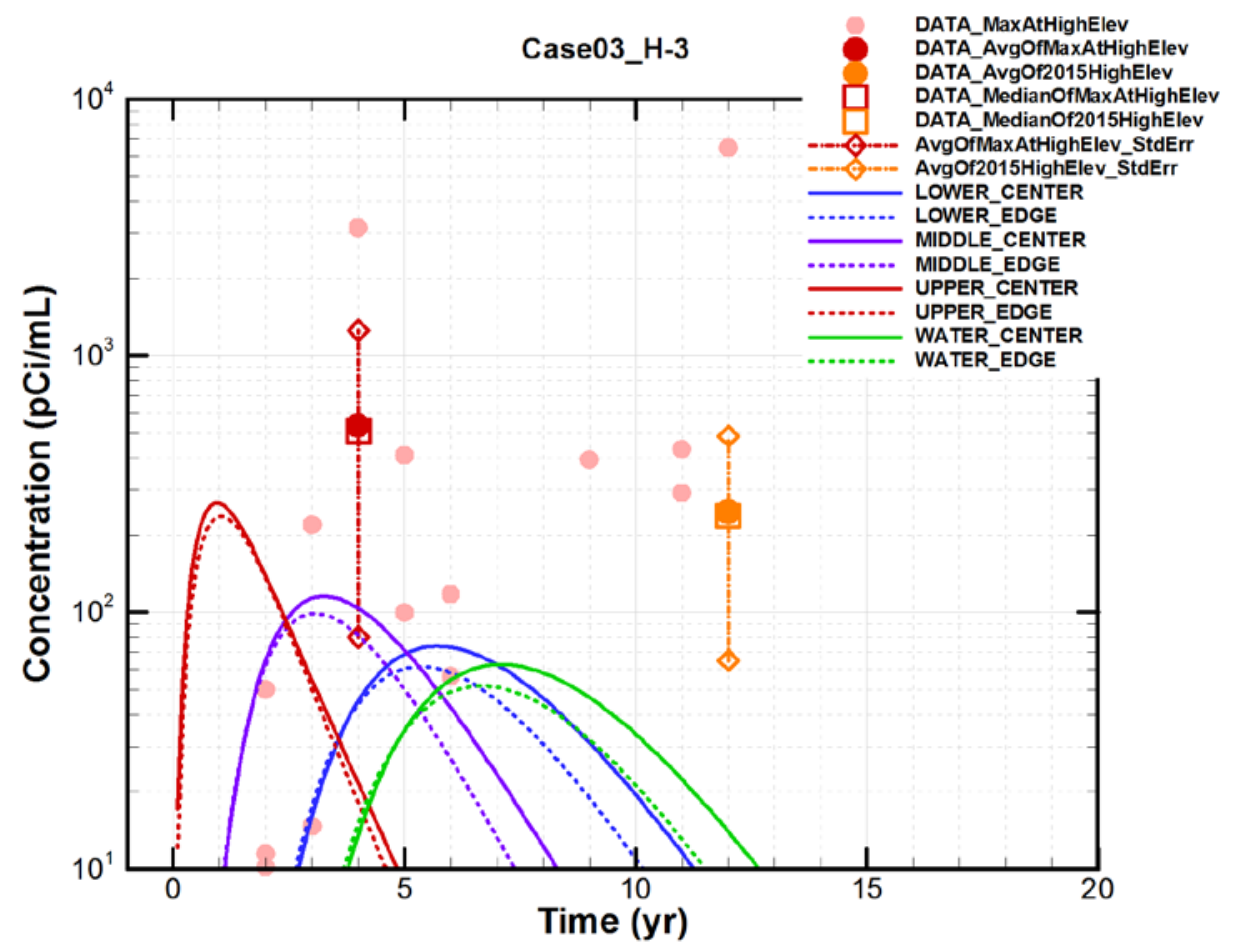

Figure 4-5. Simulated vadose zone concentrations for modeling Case03 -- B-25 boxes $+5 \%$ dispersivity.

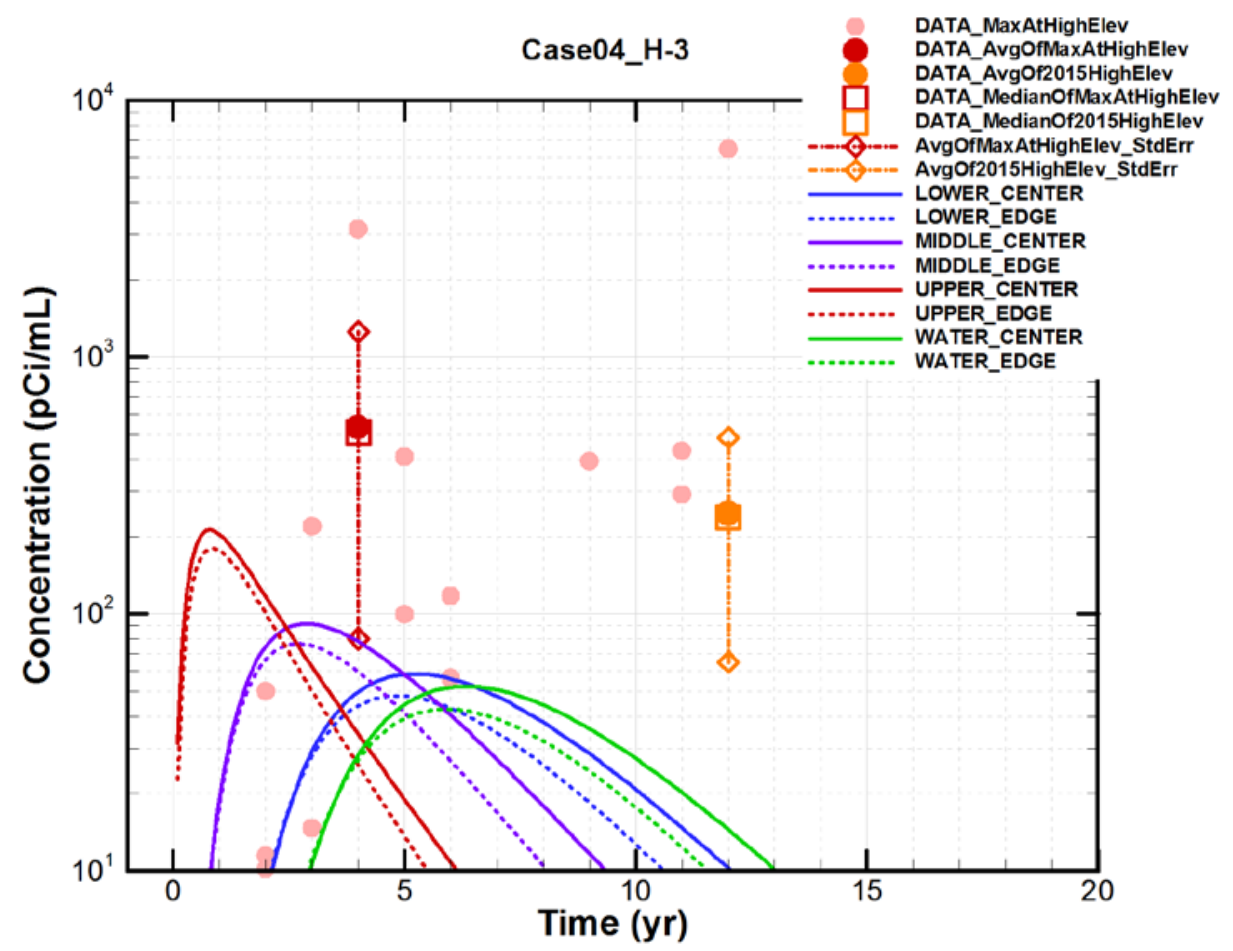

Figure 4-6. Simulated vadose zone concentrations for modeling Case04 -- B-25 boxes $+10 \%$ dispersivity. 


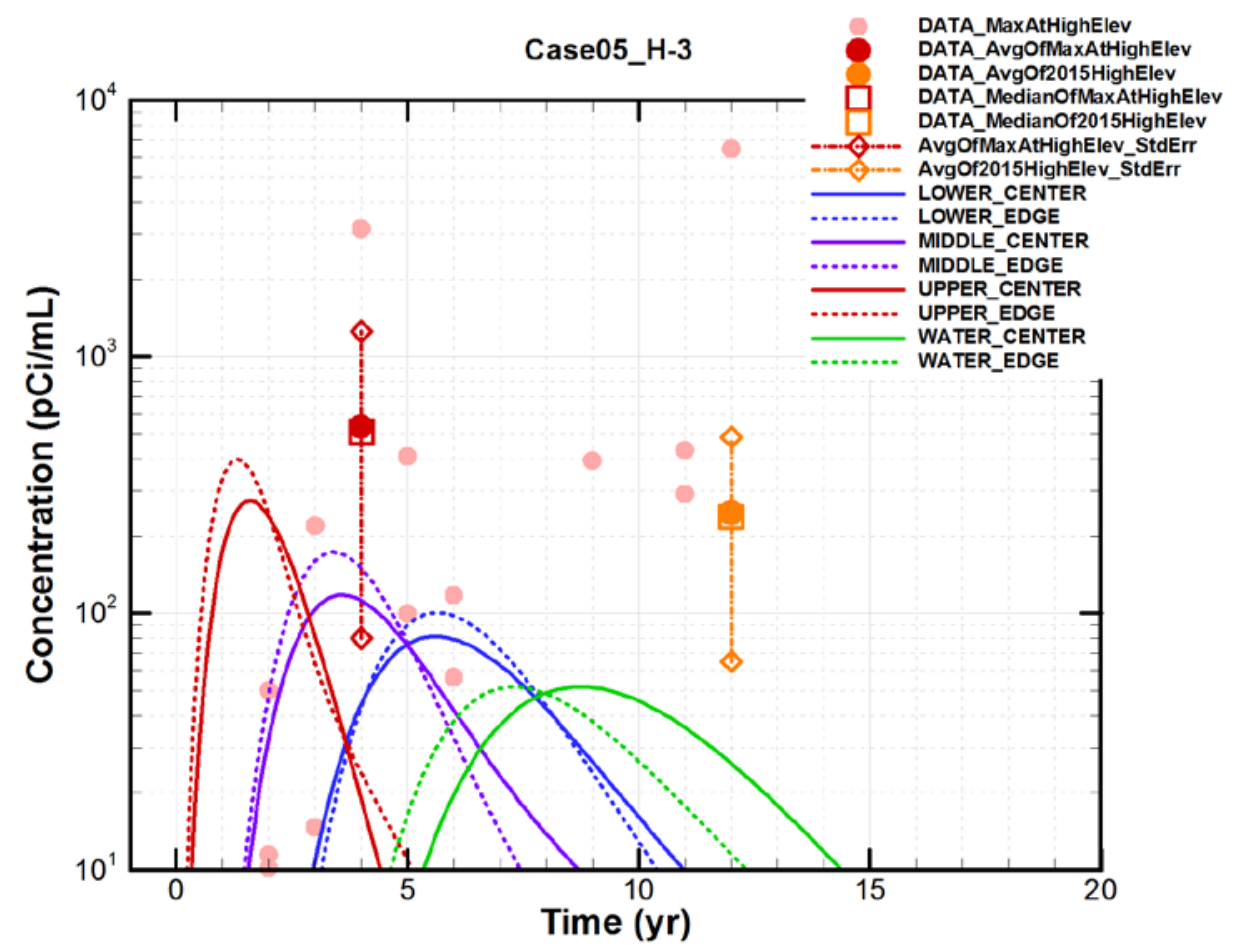

Figure 4-7. Simulated vadose zone concentrations for modeling Case05 -- B25 boxes $+25 \%$ low permeability in undisturbed soil.

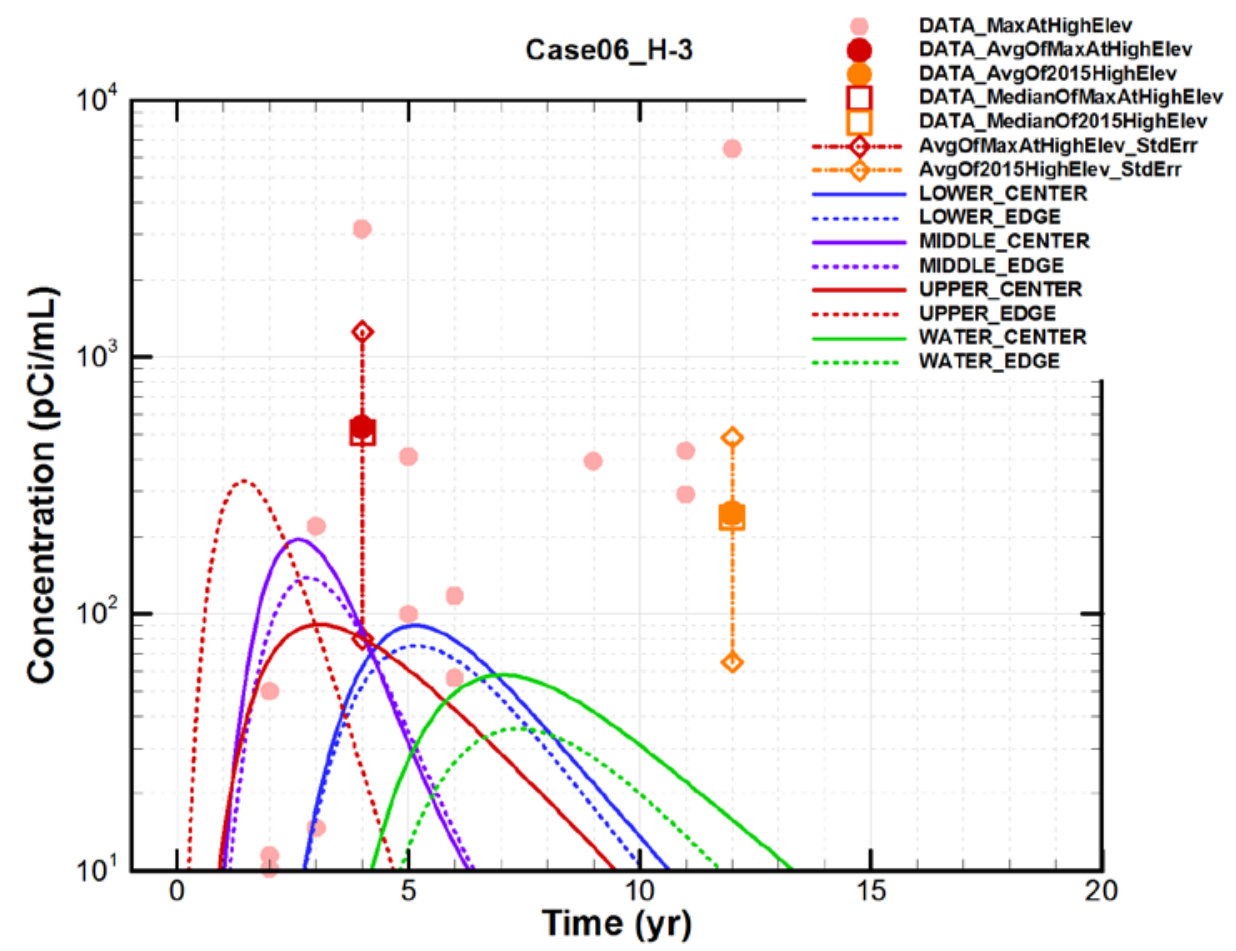

Figure 4-8. Simulated vadose zone concentrations for modeling Case06 -- B25 boxes $+35 \%$ low permeability in undisturbed soil. 
For B-25 boxes stacked four high, typical of ET1 disposal operations (Figure 2-3), Phifer and Wilhite (2001, Section 6.0) estimated a subsidence potential of $15.1 \mathrm{ft}$ out of a $17.3 \mathrm{ft}$ stack height. This subsidence potential implies $87 \%$ void space in containers not counting material porosity. Appendix A presents a derivation of equations for blending the porous-medium properties of two distinct materials to achieve the equivalent properties of the composite material. Table 4-1 and Figure 4-9 summarize the properties of the composite material. The presence of significant void space in uncompacted boxed waste disposal results in significantly lower water content (and hydraulic conductivity). The lower water content significantly raises the initial waste zone concentration, along the lines of the concentrations shown in Table 3-2.

Table 4-1. Material properties of blended backfill soil and low-density boxes.

\begin{tabular}{|c|c|c|c|c|c|}
\hline Parameter & Material 1 & Material 2 & Blend & Units & Comments \\
\hline & OSC & & & & $\begin{array}{l}\text { Operational soil cover (OSC) before dynamic } \\
\text { compaction }\end{array}$ \\
\hline & & Box & & & Box minus contents (void space) \\
\hline & & & ET waste & & $\begin{array}{l}\text { Effective properties of ET waste zone, prior to } \\
\text { compaction }\end{array}$ \\
\hline Volume fraction & 0.13 & 0.87 & 1 & & $\begin{array}{l}\text { WSRC-RP-2001-00613, Section } 6.0--15.1 \mathrm{ft} \\
\text { subsidence potential out of } 17.3 \mathrm{ft} \text { stack height } \\
\text { for B- } 25 \text { boxes }\end{array}$ \\
\hline Porosity & 0.46 & 1 & 0.9298 & & \\
\hline Solid density & 2.65 & 2.65 & 2.65 & $\mathrm{~g} / \mathrm{mL}$ & \\
\hline Bulk density & 1.431 & 0 & 0.18603 & $\mathrm{~g} / \mathrm{mL}$ & \\
\hline Saturated hydraulic & $3.8 \mathrm{E}+03$ & 0 & $4.94 \mathrm{E}+02$ & $\mathrm{~cm} / \mathrm{yr}$ & Box void space assumed to be impermeable \\
\hline conductivity & $1.2 \mathrm{E}-04$ & $0.0 \mathrm{E}+00$ & $1.6 \mathrm{E}-05$ & $\mathrm{~cm} / \mathrm{s}$ & \\
\hline
\end{tabular}

The five additional modeling cases using the waste zone properties shown in Table 4-1 and Figure 4-9 are summarized as follows:

- Case02: $\mathrm{B}-25$ box + dispersivity $=0 \%$ of plume travel distance

- Case03: B-25 box + dispersivity $=5 \%$ of plume travel distance

- Case04: B-25 box + dispersivity $=10 \%$ of plume travel distance

- Case05: B-25 box + 25\% low-permeability soil beneath waste zone

- Case06: B-25 box + 35\% low-permeability soil beneath waste zone

All five cases have an initial concentration in the waste zone of $873 \mathrm{pCi} / \mathrm{mL}$ for an ET1 tritium inventory of $2.21 \mathrm{Ci}$. Case02 maximizes the peak concentration at downstream lysimeter locations by minimizing plume dispersion. The peak simulated concentrations at the shallow Upper lysimeters are around 400 $\mathrm{pCi} / \mathrm{mL}$, which is similar to the mean and median values given in Table 3-3 and well within the 95\% confidence bounds, unlike Case01. However, the Case02 plume has a short duration, approximately three years, whereas the VZMS data suggest a broader plume (Figure 3-2). Figure 4-10 provides snapshots of the plume at elapsed times of $0.4,1.0$ and 1.6 years. 


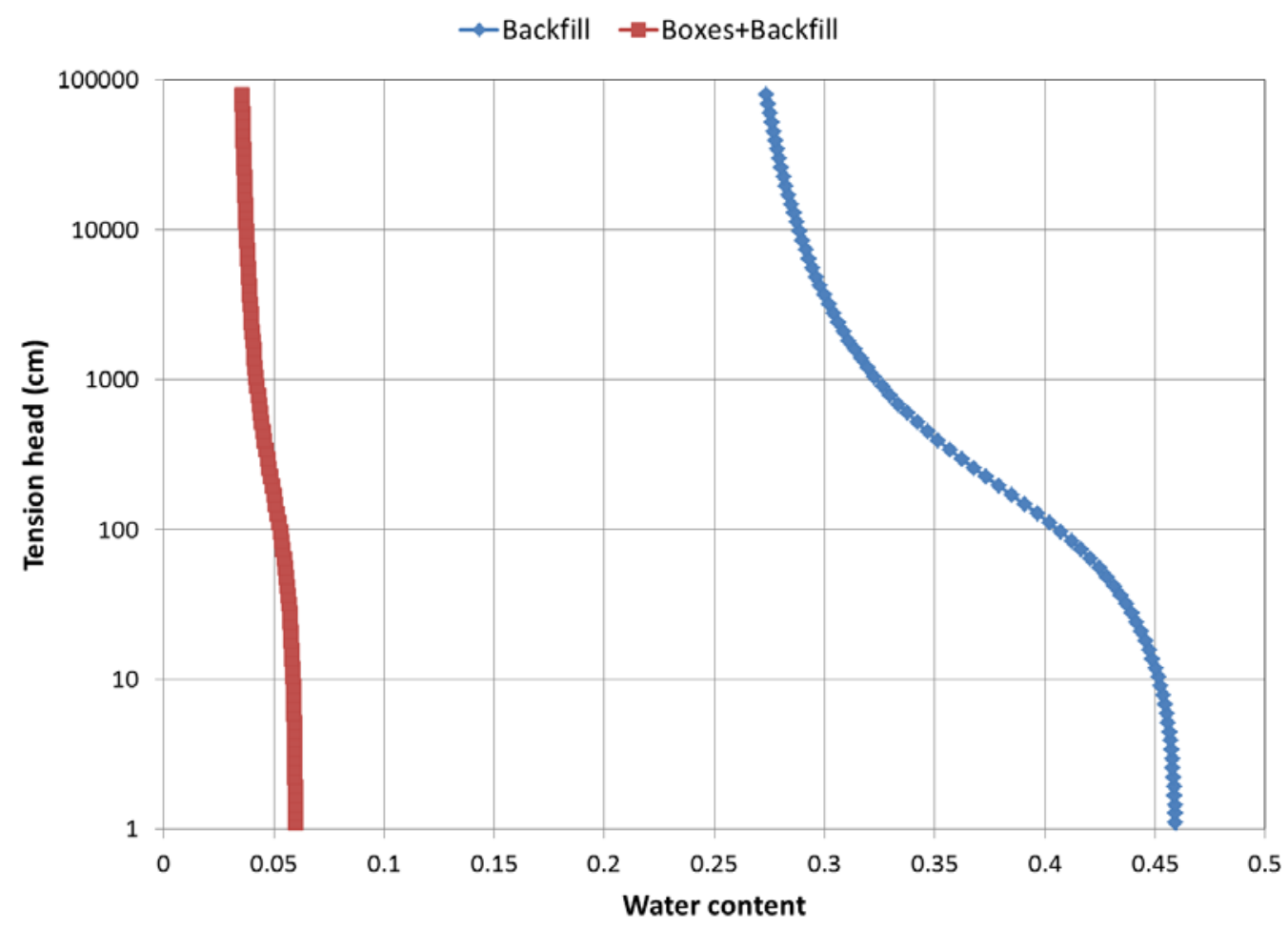

(a)

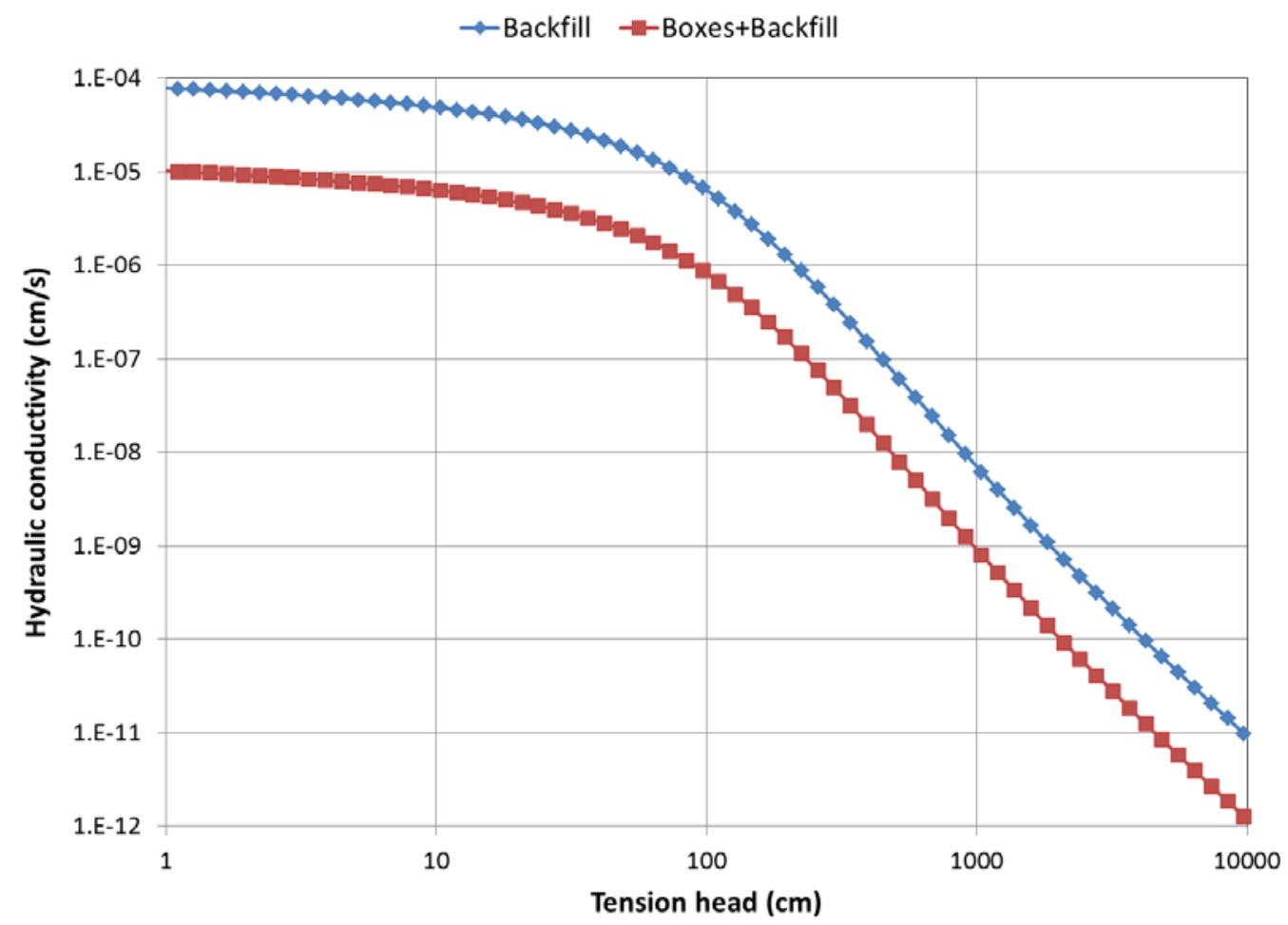

(b)

Figure 4-9. Soil characteristics curves for mixed backfill soil and uncompacted boxed waste. 
C_ 0.40

pCi/mL

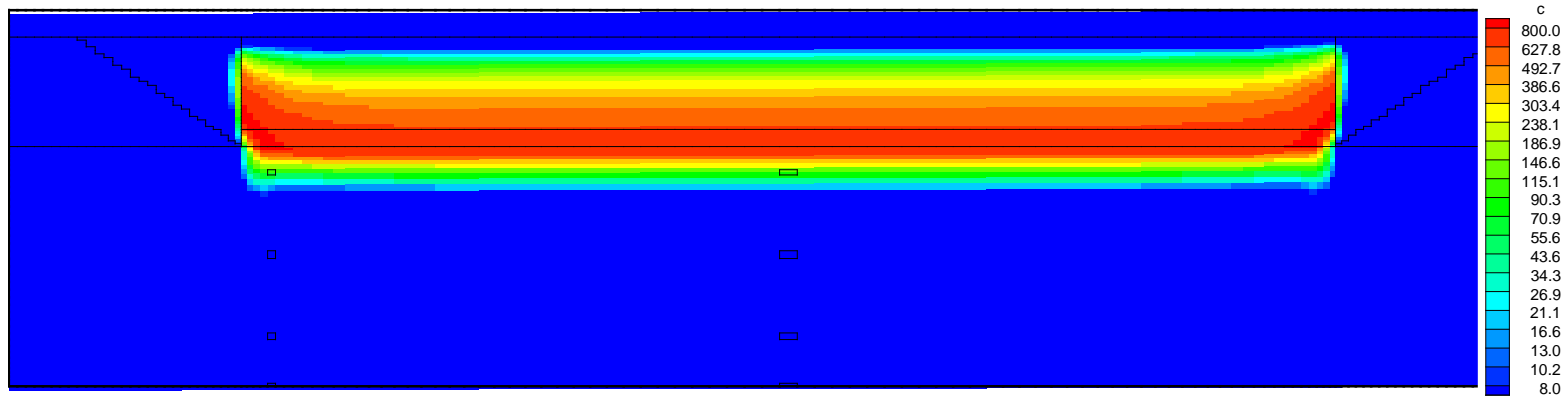

(a)

C_1.00

pCi/mL

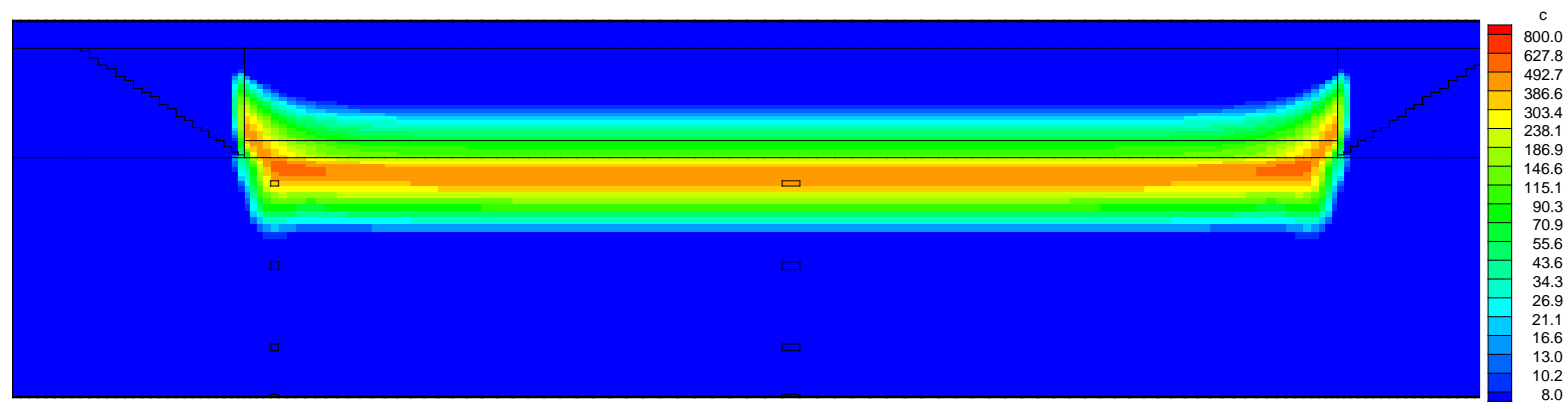

(b)

C_1.60

pCi/mL

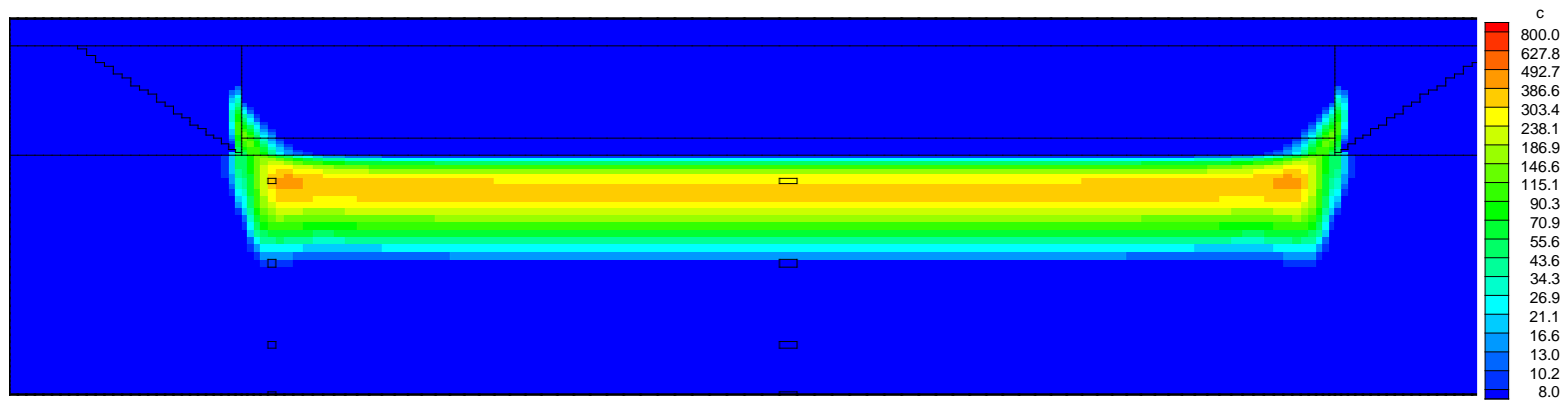

(c)

Figure 4-10. Simulated H-3 plume for Case02 at an elapsed times of (a) 0.4 yr, (b) $1.0 \mathrm{yr}$, and (c) 1.6 yr.

At the expense of reducing peak concentration, the plume duration can be expanded by introducing dispersion, which is expected due to heterogeneity. Case03 and Case04 introduce dispersion in the form of a non-zero dispersivity ranging from $5 \%$ to $10 \%$ of plume travel distance, taken as $35 \mathrm{ft}$. A dispersity of $10 \%$ is a common assumption (Zheng and Bennett 1995, Section 9.3.2). Case05 and Case06 create similar plume spreading by introducing explicit heterogeneity through a bi-modal permeability distribution, where the low-permeability fraction ranges from 25\% to 35\% (Figure 4-11). Heterogeneity creates a non-uniform velocity field (Figure 4-12) that results in portions of the plume moving at a different rate than the average. 


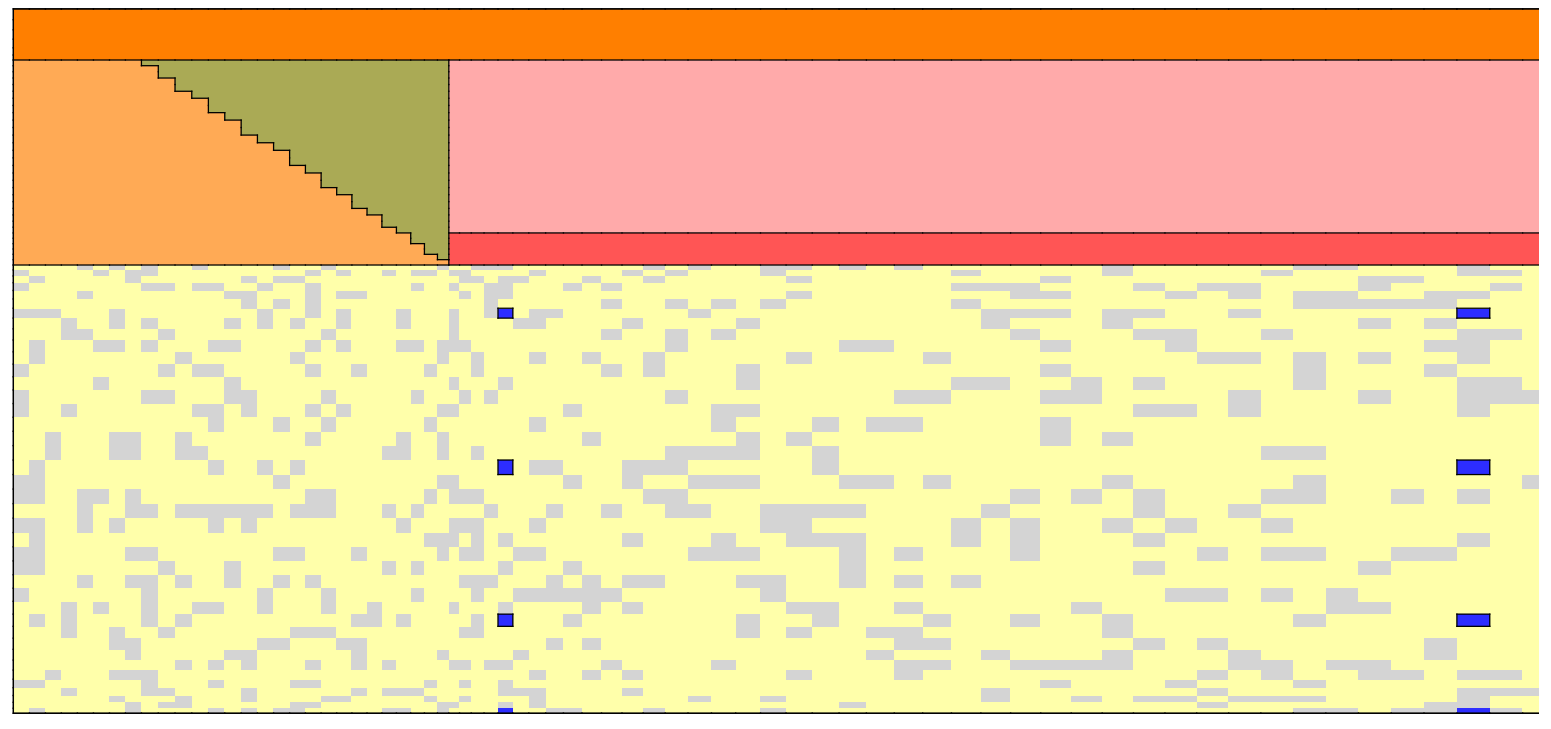

(a)

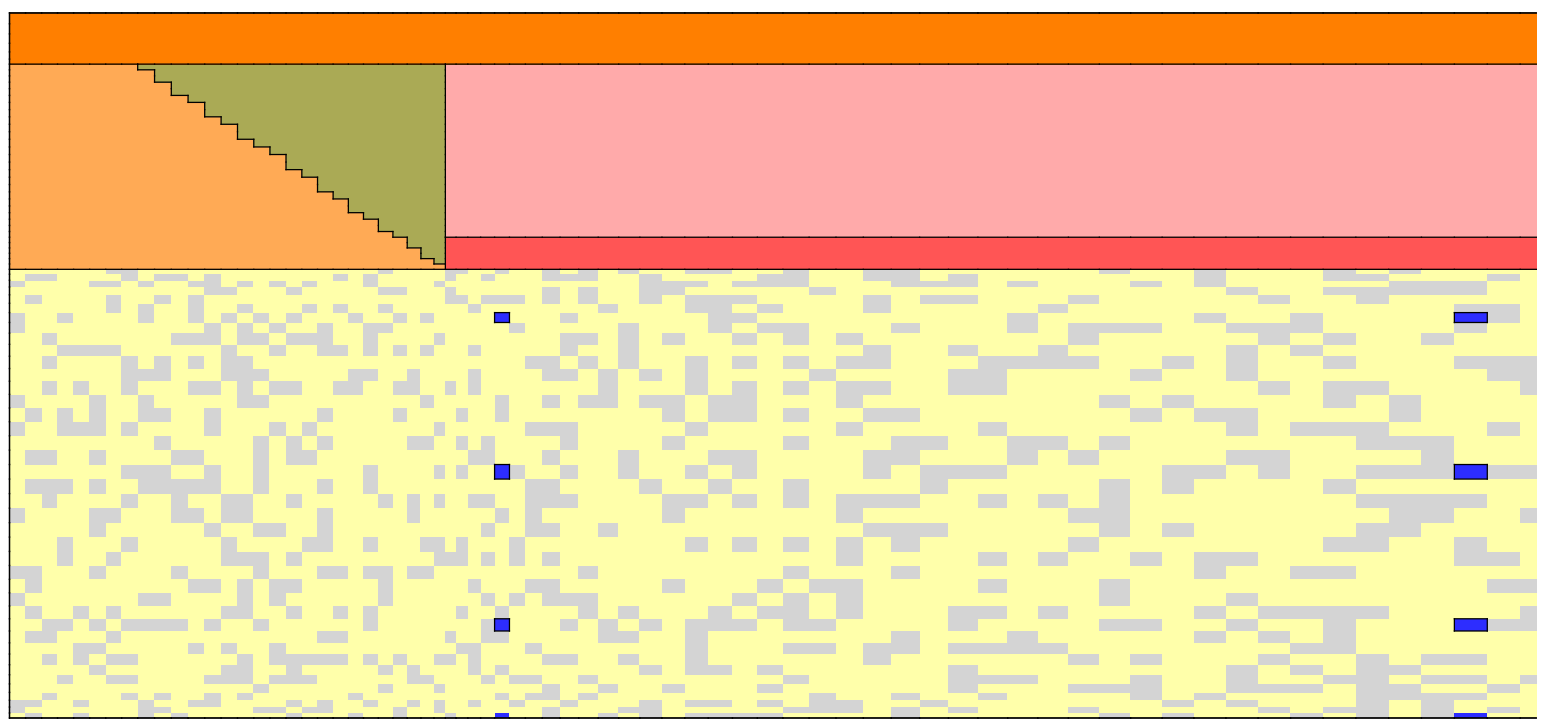

(b)

Figure 4-11. Random bi-modal hydraulic conductivity fields with low-permeability fractions of (a) $25 \%$ and (b) $35 \%$. 
Case05

s: $\begin{array}{lllllllllllllllllll}0.05 & 0.1 & 0.15 & 0.2 & 0.25 & 0.3 & 0.35 & 0.4 & 0.45 & 0.5 & 0.55 & 0.6 & 0.65 & 0.7 & 0.75 & 0.8 & 0.85 & 0.9 & 0.95\end{array}$

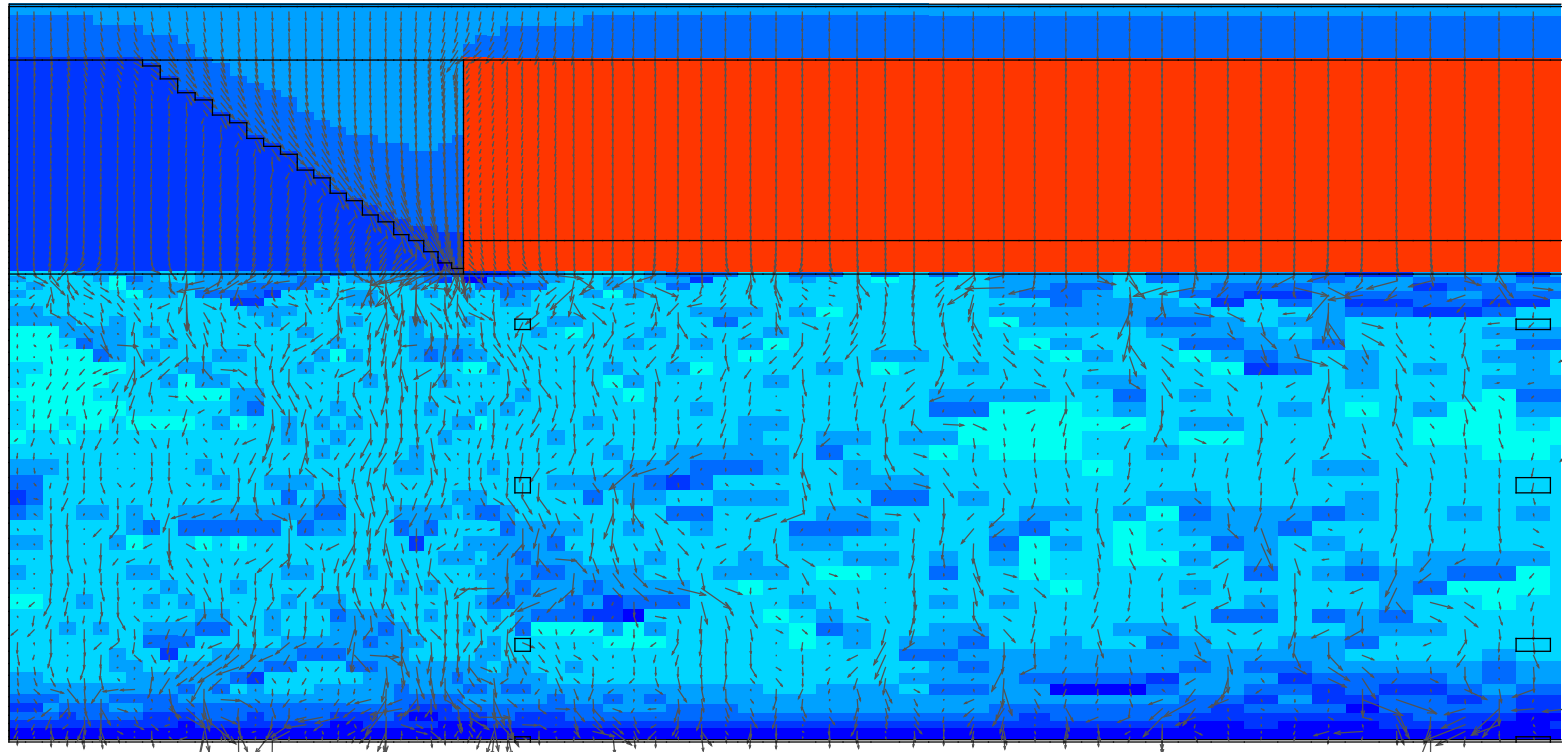

(a)

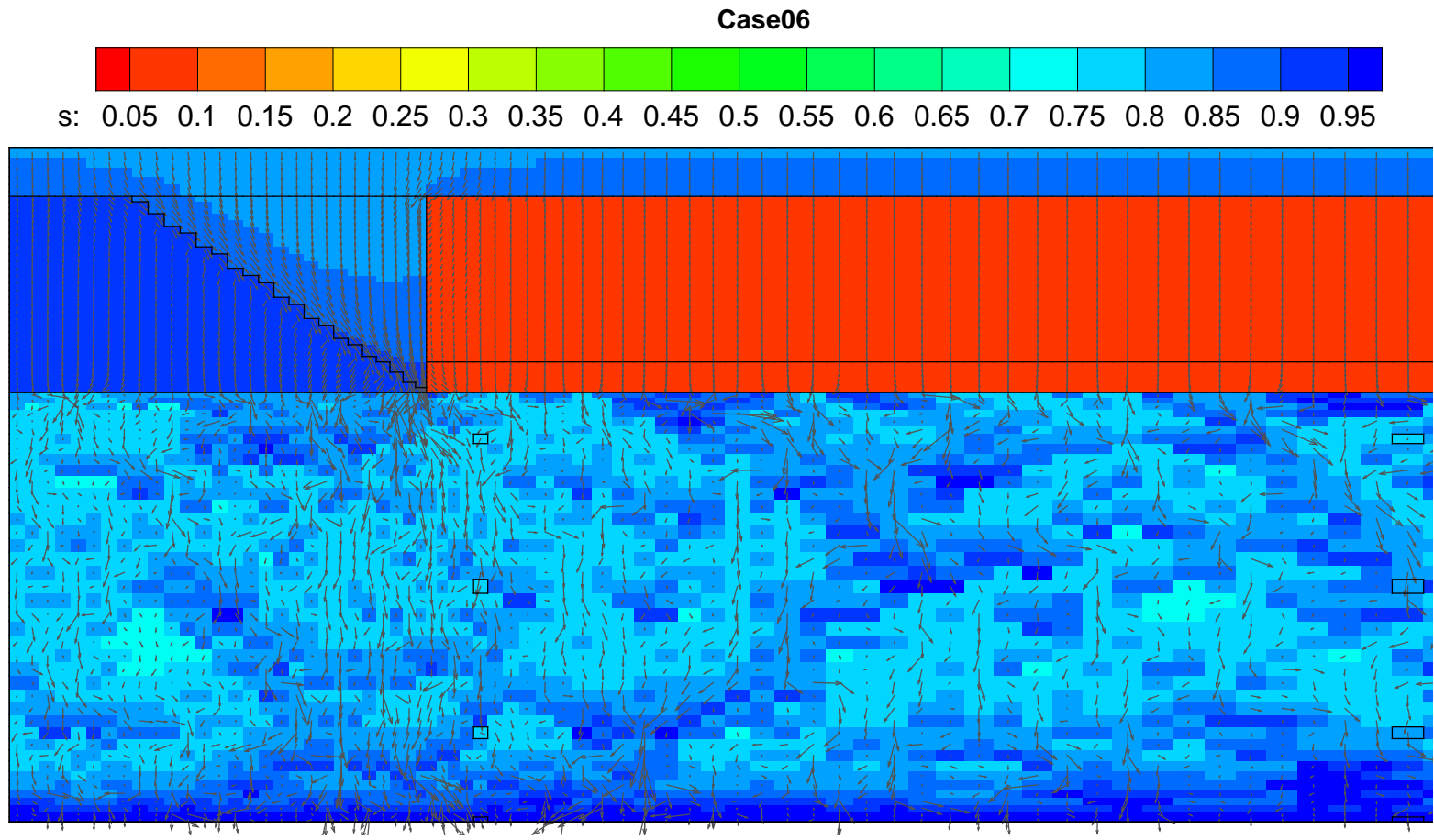

(b)

Figure 4-12. Flow fields resulting from low-permeability fractions of (a) $25 \%$ and (b) $35 \%$. 
Cases03 through Case06 exhibit broader breakthrough curves with lower peaks generally in the range of 100 to $300 \mathrm{pCi} / \mathrm{mL}$ for the Upper lysimeters (Figure 4-5 through Figure 4-8). Table 4-2 tabulates peak concentrations for both the Upper and Lower lysimeter pairs, and defines a representative value for the Edge and Center pair at each elevation. For Cases01 through Case04, the maximum of the Center and Edge concentrations is taken as the Representative value. For Case05 and Case06 the average value is taken because simulated concentration exhibits significant variability due to the modeled heterogeneity. Case03 through Case06 are considered more realistic than the no-dispersion Case02. The averages for these cases are 257 and $76 \mathrm{pCi} / \mathrm{mL}$ for the Upper and Lower simulated lysimeter elevations, respectively.

Table 4-2. Simulated peak H-3 concentrations at UPPER and LOWER lysimeter locations.

\begin{tabular}{||l|c|c|c|c|c|c|c||}
\hline $\begin{array}{l}\text { Peak H-3 Concentration } \\
\text { from Simulated Lysimeters }\end{array}$ & Case01 & Case02 & Case03 & Case04 & Case05 & Case06 & $\begin{array}{c}\text { Case03 to } \\
\text { Case06 average }\end{array}$ \\
\hline UPPER_CENTER & 107 & 411 & 267 & 213 & 275 & 91 & \\
\hline UPPER_EDGE & 102 & 363 & 238 & 180 & 399 & 331 & \\
\hline Representative UPPER & 107 & 411 & 267 & 213 & 337 & 211 & 257 \\
\hline LOWER_CENTER & 66 & 114 & 74 & 59 & 81 & 90 & \\
\hline LOWER_EDGE & 61 & 90 & 61 & 48 & 101 & 75 & \\
\hline Representative LOWER & 66 & 114 & 74 & 59 & 91 & 83 & 76 \\
\hline
\end{tabular}

While the Vadose Zone Monitoring System is focused on tritium, the overarching issue, which will be discussed in Section 6.0, is whether ET1 can be expected to meet performance objectives, considering contributions to the sum-of-fractions from all radionuclides disposed of in ET1. The other species indicated explicitly in Figure 2-1 are slow decaying relative to tritium and similarly mobile. The preceding vadose zone simulation cases were repeated for a non-decaying tracer species, which is used as surrogate for C-14, I-129, Nb-94 and Tc-99. However, vadose zone tracer simulation results are not explicitly presented, because they are similar to the $\mathrm{H}-3$ results already presented.

\subsection{Saturated zone model simulations}

Four aquifer scenarios were considered, where " $\mathrm{x}$ ” represents the last digit of a vadose zone case:

- Case0x: Uniform waste distribution + instantaneous disposal (PA case)

- Case1x: As-disposed-of ET1 waste distribution + instantaneous disposal

- Case2x: Uniform waste distribution + 10 year ET2 delay

- Case3x: As-disposed-of ET1 waste distribution + 10 year ET2 delay (best-estimate case)

All simulations were performed using the 2008 PA aquifer model (WSRC 2008), which analyzed ET1 and ET2 together as a disposal unit group. Subsequent Special Analysis modifications described by Swingle (2012) were not adopted in this study because the original PA model is suitable for assessing relative differences in modeling scenarios.

A 10 year difference in waste disposal times between ET1 and ET2 was assumed based on the volume consumption histories summarized in Figure 2-2 and Table 4-3. ET1 opened in 2001 and reached 25\% volume consumption in 2002, 50\% in early 2003, 75\% in 2005, 88\% in 2006, 99\% in 2011 and 99.5\% in 2015. ET2 opened in 2004 and reached 25\% volume consumption in 2007, 50\% in early 2011, 75\% in 2014, and 76\% in 2016. The 50\% volume consumption (or median) dates for ET1 and ET2 differ by 8 years. ET2 disposals have slowed with the opening of ET3 and are expected to continue for several years past the 75\% consumption date. The average disposal dates for ET1 and ET2 are thus expected to differ by more than 8 years, approximately 10 years as assumed in modeling herein. 
Table 4-3. Disposal volume history for ET2 from WITS.

\begin{tabular}{||c|c||}
\hline Date & Volume Consumption \\
\hline June 2004 (trench opened) & $0 \%$ \\
\hline November 2007 & $25 \%$ \\
\hline March 2011 & $50 \%$ \\
\hline September 2014 & $75 \%$ \\
\hline October 2016 (current) & $76 \%$ \\
\hline
\end{tabular}

Simulated tritium and tracer concentrations are provided in Figure 4-13 through Figure 4-20. Scenario 2x produces a double peak reflecting the 10 year delta in disposal times between ET1 and ET2. The double peak largely disappears in Scenario 3x because ET1 waste is placed in the upgradient half of the disposal unit footprint, which compensates for the time delay in uniform ET2 burials. As such, the plumes emanating from ET1 and ET2 arrive at the 100 meter perimeter much closer in time compared to Scenario $2 \mathrm{x}$.

Table 4-4 recomputes the WITS SOF for each aquifer and vadose modeling scenario, based on the relative differences in the peak concentrations from Figure 4-13 through Figure 4-20. The most realistic cases are considered to be those four in the Case33 through Case36 series. These cases consider B-25 box disposal and incorporate a realistic level of plume dispersion due to heterogeneity in soil properties (or waste release). The adjusted WITS SOF for these cases averages 0.89 compared to an actual WITS SOF of 0.87 (Figure 2-1). A second calculation considers the hypothetical possibility that the actual buried inventory of tritium (alone) is higher than that recorded in WITS. This calculation indicates that the tritium inventory could be up to $50 \%$ higher without exceeding an SOF of 1.0 for the average of Case33 through Case36. 


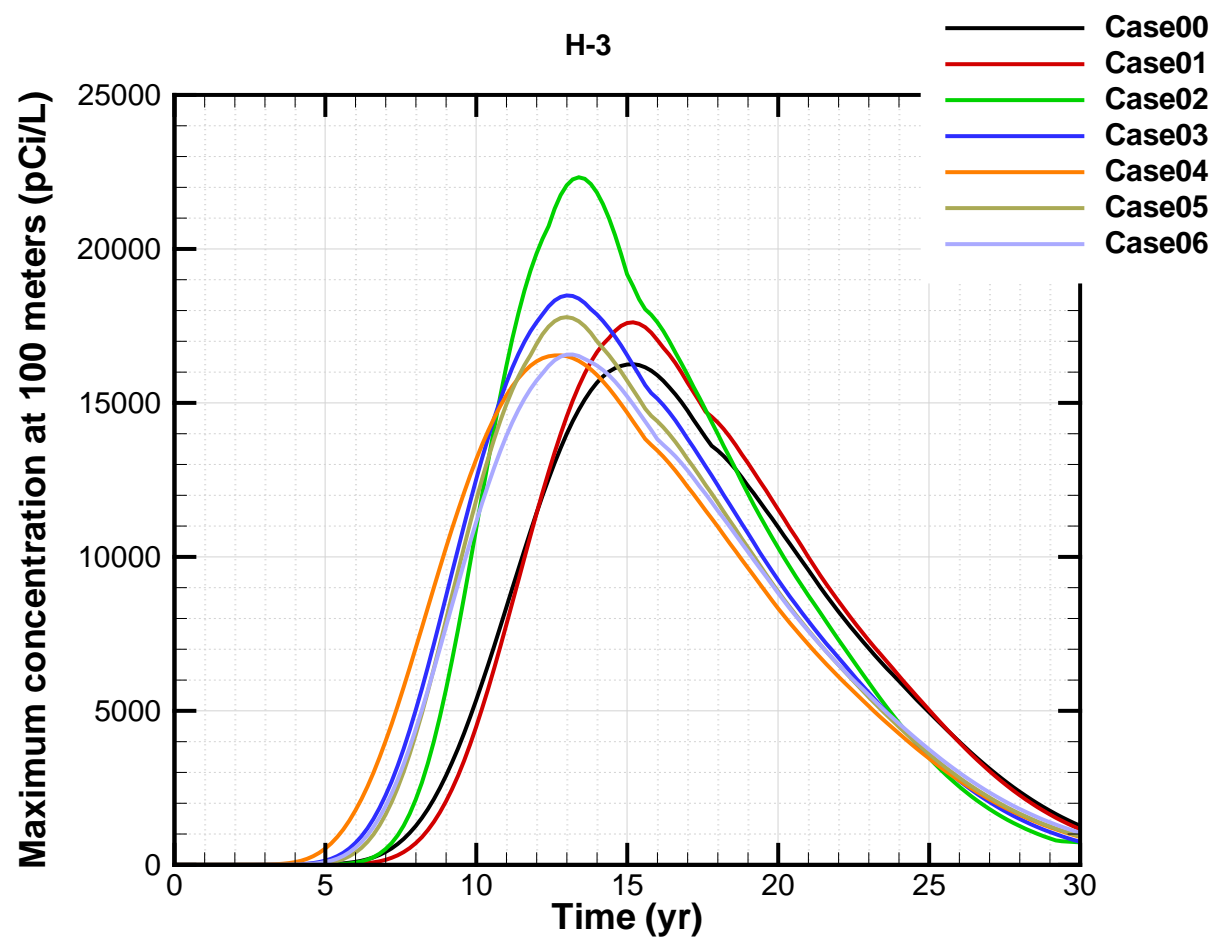

Figure 4-13. Simulated tritium concentration for aquifer scenario $0 x$.

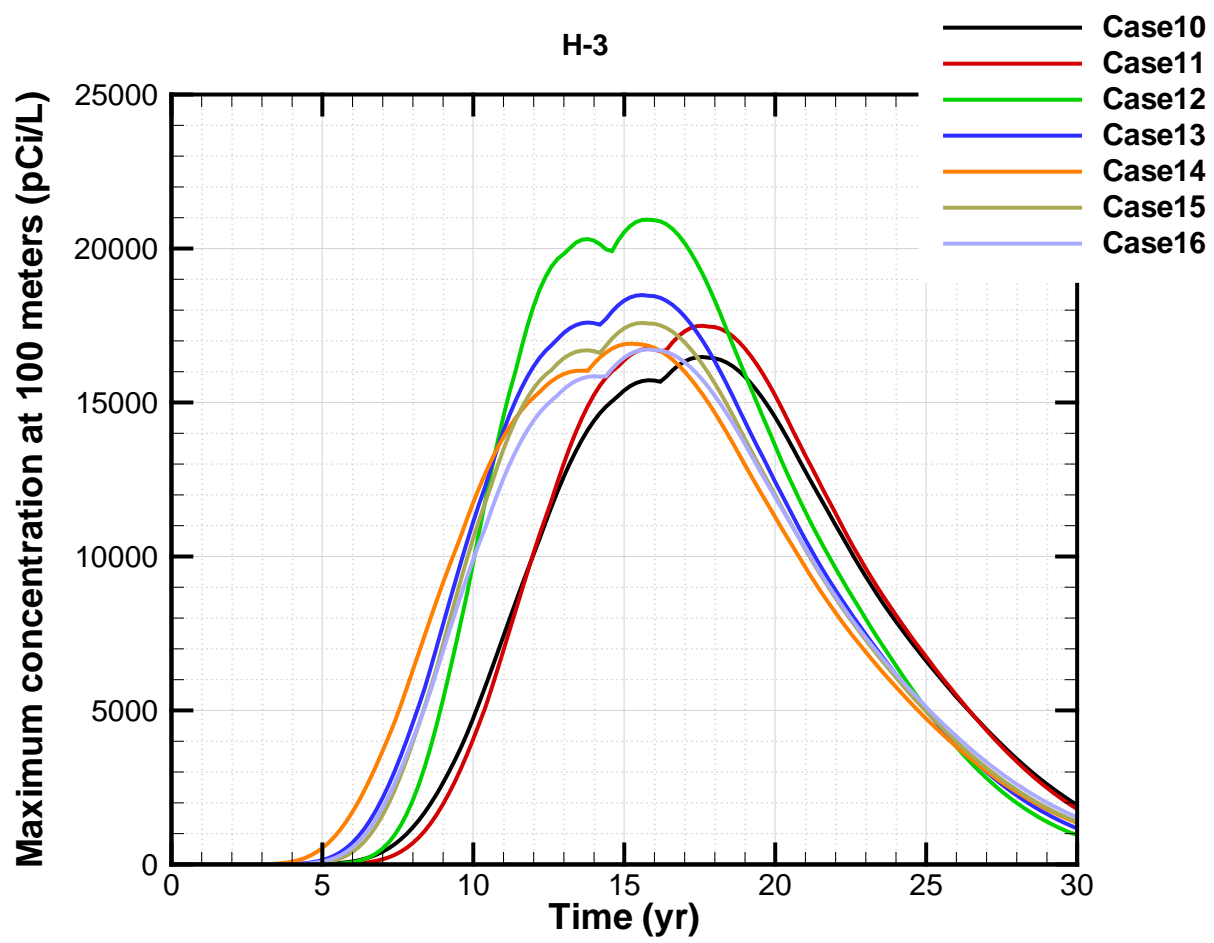

Figure 4-14. Simulated tritium concentration for aquifer scenario 1x. 


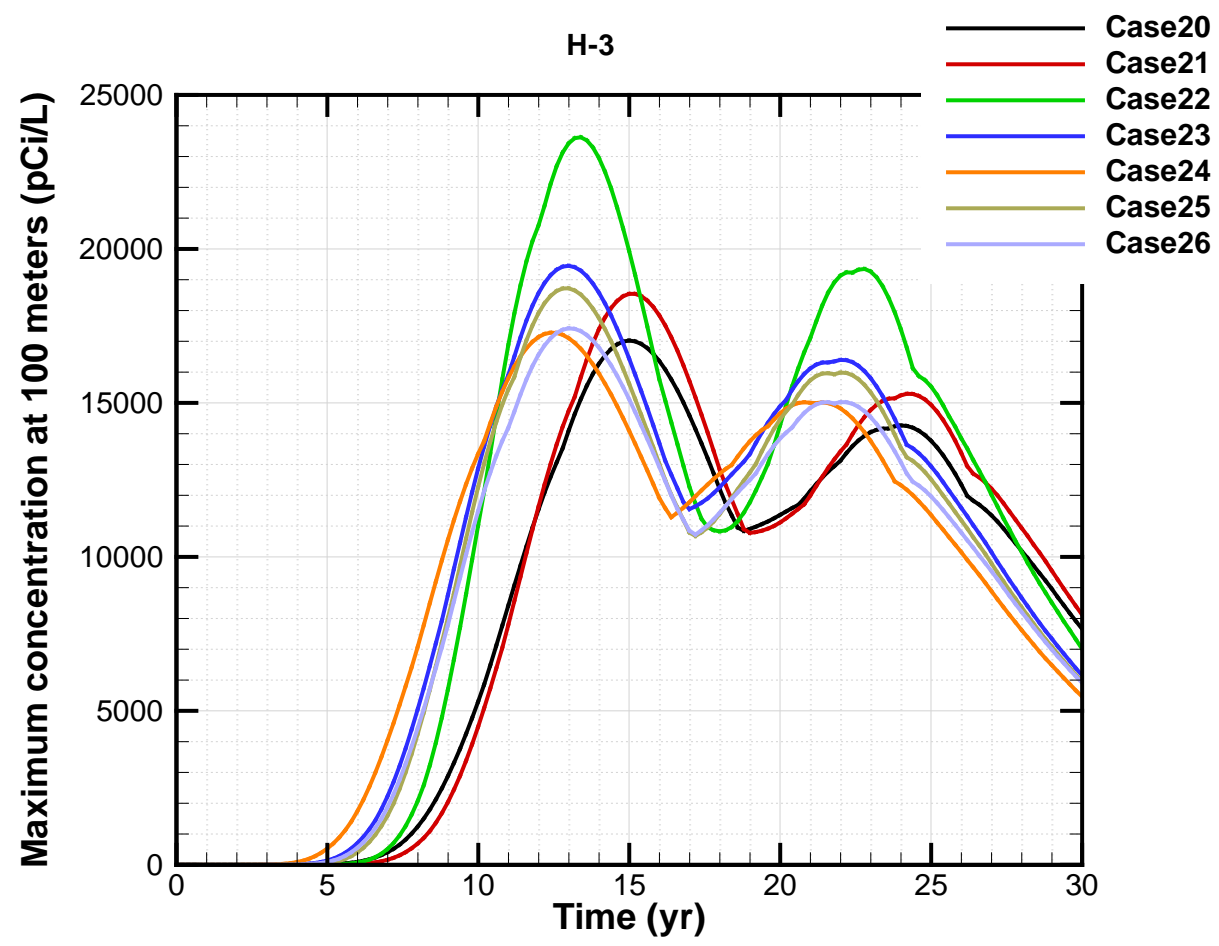

Figure 4-15. Simulated tritium concentration for aquifer scenario $2 x$.

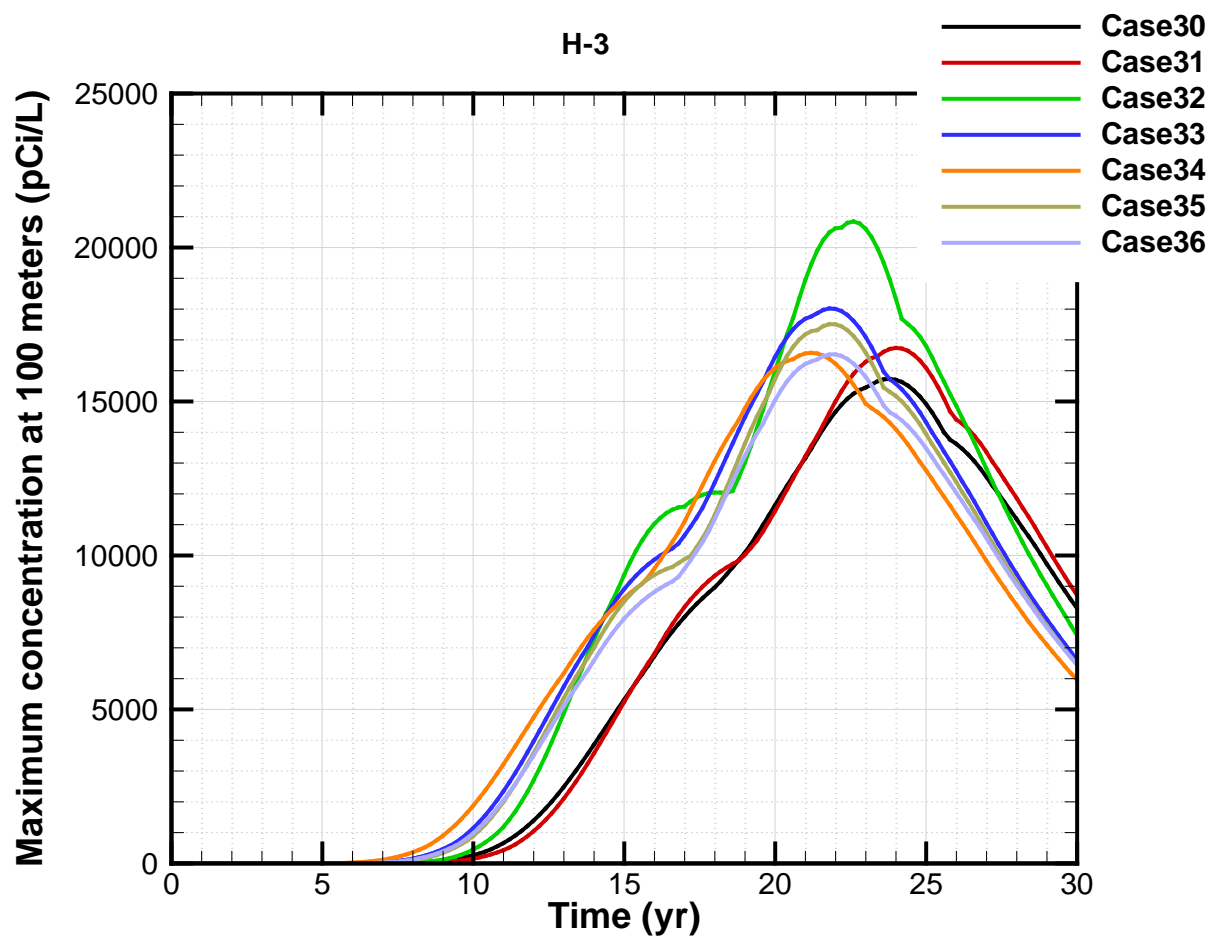

Figure 4-16. Simulated tritium concentration for aquifer scenario $3 x$. 


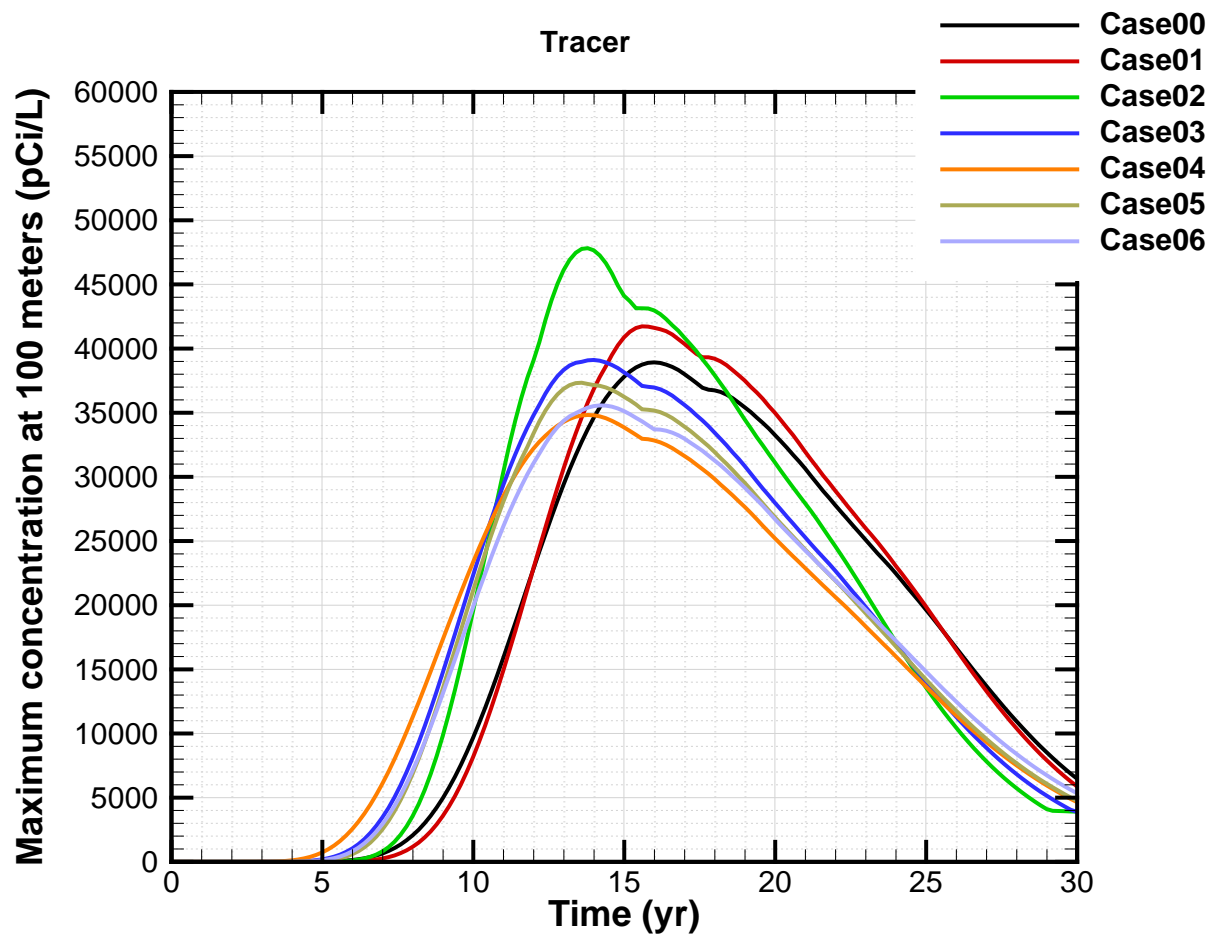

Figure 4-17. Simulated tracer concentration for aquifer scenario $0 x$.

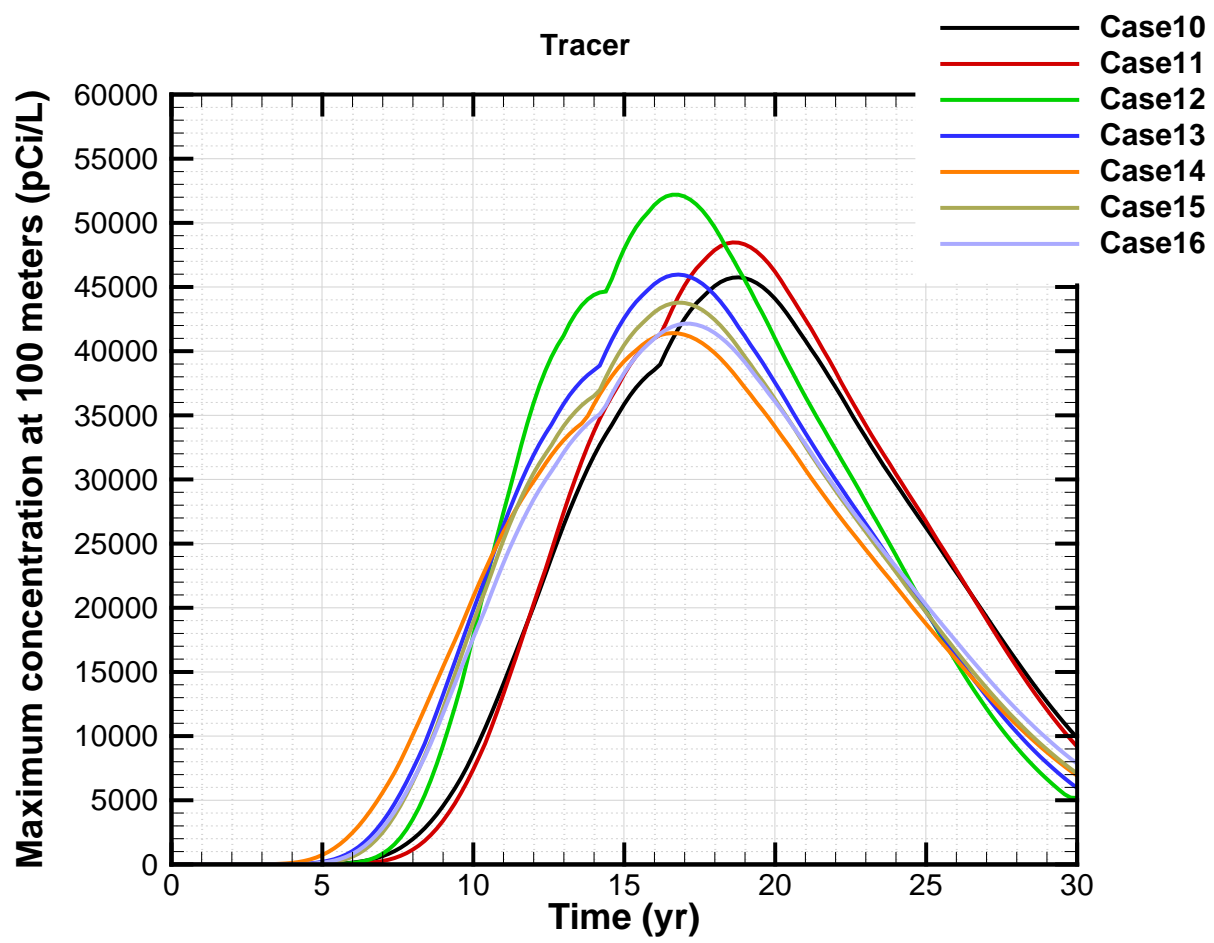

Figure 4-18. Simulated tracer concentration for aquifer scenario 1x. 


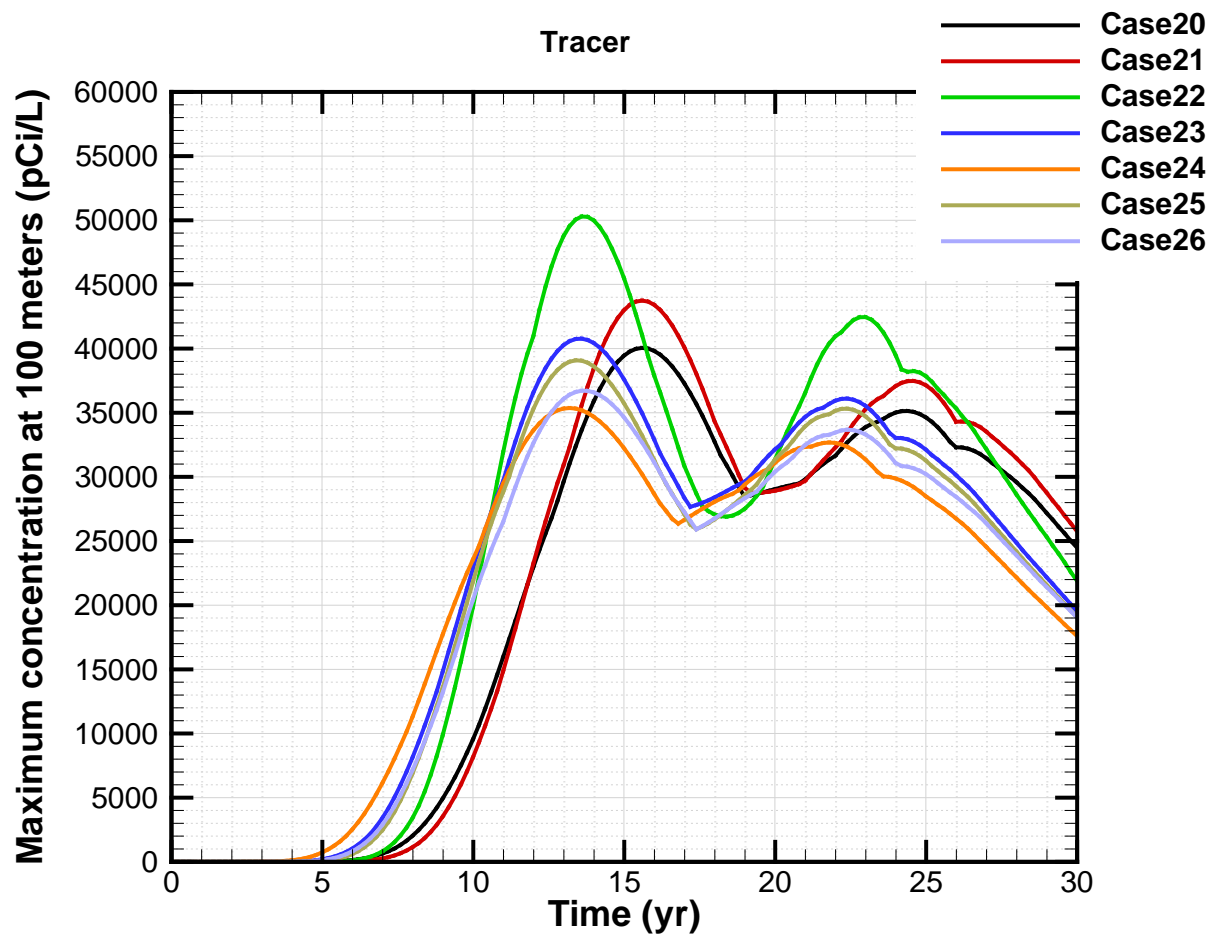

Figure 4-19. Simulated tracer concentration for aquifer scenario $2 x$.

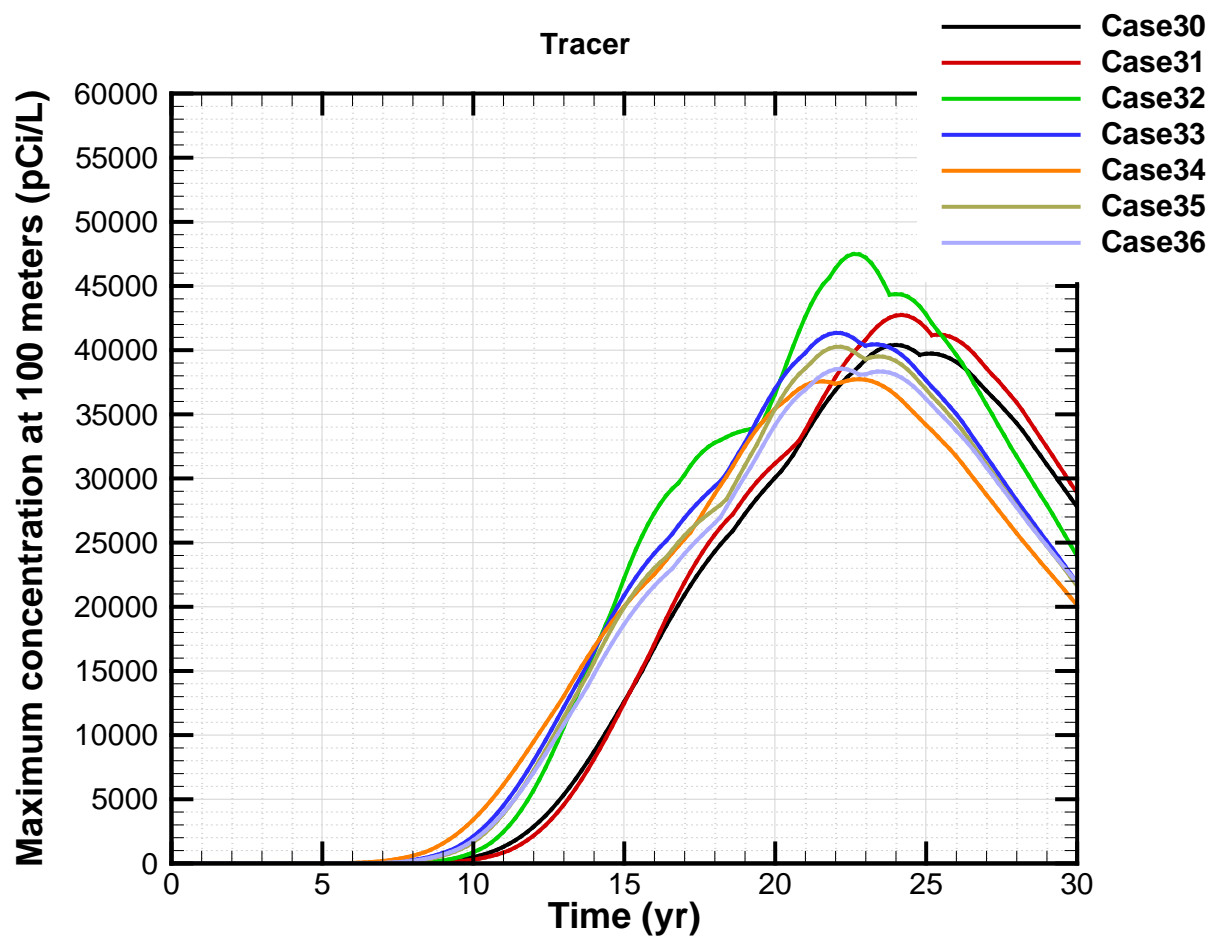

Figure 4-20. Simulated tracer concentration for aquifer scenario 3x. 
Table 4-4. Projected WITS sum-of-fractions for aquifer scenarios $0 x$ through $3 x$.

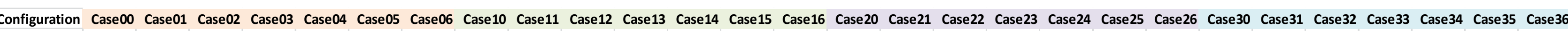
Instantaneous disposals in ET1 and ET2 As-disposed-of timing in ET1 and ET

Uniform ET1 waste distribution

As-disposed-of ETT waste distibution

$$
\text { B-25 waste containers }
$$

B-25 waste containers

Hoterogeneous soil: $25 \%$ low permeability

Heterogeneous soil: $35 \%$ low permeability

Dispersivity $=0 \mathrm{ft}$

Dispersivity $=1.75 \mathrm{ft}(5 \%$ of $35 \mathrm{ft})$

vity $=3.5 \mathrm{ft}(10 \%$ of $35 \mathrm{ft})$

Vadose zone model description: PA

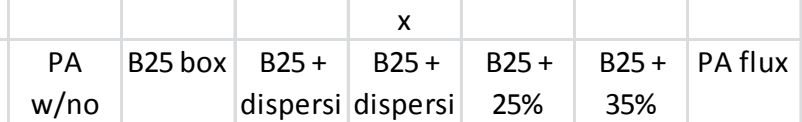

$\begin{array}{llllll}\text { waste } & \text { on }= & \text { on }= & \text { low } & \text { low } \\ \text { in side } & 5 \% \mathrm{~L} & 10 \% \mathrm{~L} & \text { perm. } & \text { perm. }\end{array}$

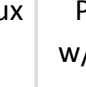

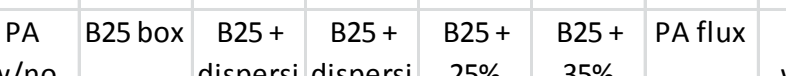

$\begin{array}{lllll} & \text { DAspersi dispersi } & 25 \% & 35 \% \\ \text { Who } & & \end{array}$

\begin{tabular}{lll|l} 
on $=$ & on $=$ & low & low \\
$5 \%$ & $10 \%$ & perm &
\end{tabular}

$w / n$

B25 box

Performance Objective Case00 Case01 Case02 Case03 Case04 Case05 Case06 Case10 Case11 Case12 Case13 Case14 Case15 Case16 Case20 Case21 Case22 Case23 Case24 Case25 Case26 Case30 Case31 Case32 Case33 Case34 Case35 Case36

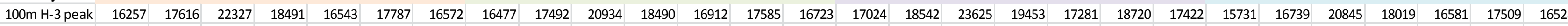

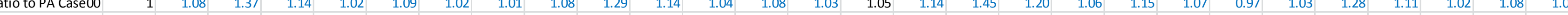

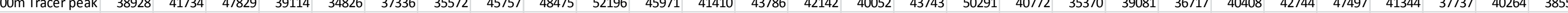
WITS inventory

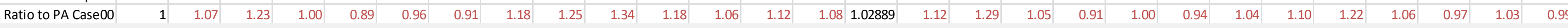

osed Sum of Fractions $\quad$ WITS Projected

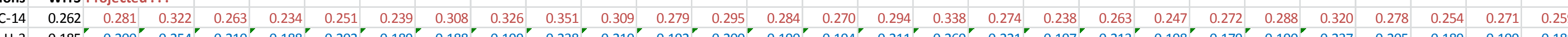

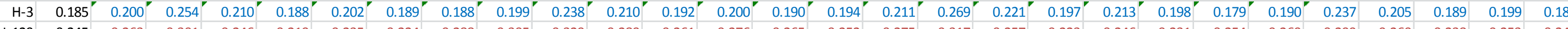

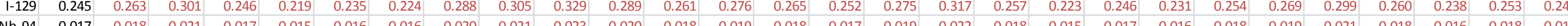

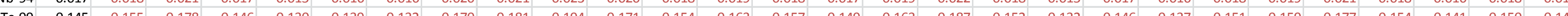
sof 0.854

Ratio to WITS SOF $0.87 \quad 0.93 \quad 1.09$ 1

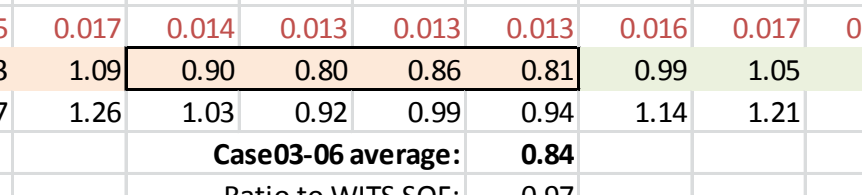

Case03-06 average: $\quad 0.84$
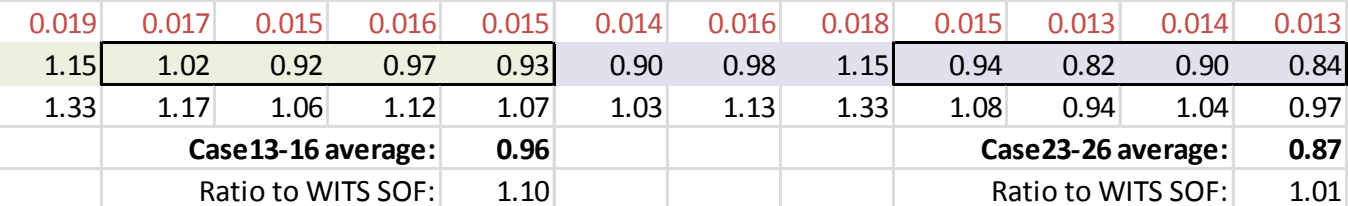

$\begin{array}{lll}0.015 & 0.015 & 0.017\end{array}$

Case13-16 average:

Ratio to WITS SOF

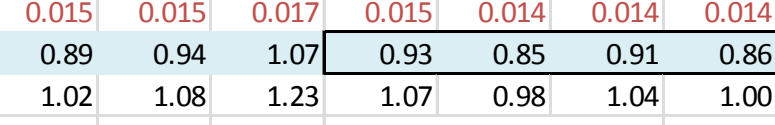
1.5

Tritium multiplier

As-disposed Sum of Fractions WITS Projected....

$\begin{array}{lllll}1.5 & 1.5 & 1.5 & 1.5 & 1.5\end{array}$

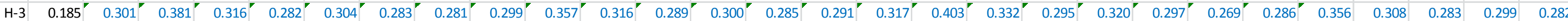

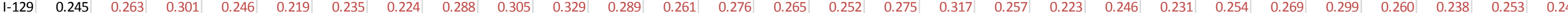
\begin{tabular}{lllllllllllllllllllllllllllllllll}
$\mathrm{Nb}-94$ & 0.017 & 0.018 & 0.021 & 0.017 & 0.015 & 0.016 & 0.016 & 0.020 & 0.021 & 0.023 & 0.020 & 0.018 & 0.019 & 0.018 & 0.017 & 0.019 & 0.022 & 0.018 & 0.015 & 0.017 & 0.016 & 0.018 & 0.019 & 0.021 & 0.018 & 0.016 & 0.018 & 0.017 \\
\hline
\end{tabular}

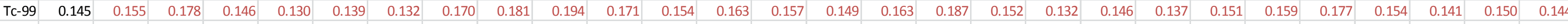
sof 0.854 


\subsection{Model uncertainty analysis}

Revising the ET1 conceptual model to incorporate boxes in the waste zone significantly improved agreement with the VZMS data. However, all of the modeling cases yield a peak $\mathrm{H}-3$ concentration somewhat below the sample mean and bootstrapping median values, which may point to other model biases. One candidate is the hydraulic properties for native sediments, which may overpredict saturation and thus underpredict concentration. Simulated saturation in the Lower Vadose Zone sediment is 73\%. Supporting the hypothesis of a model bias is Section 5.8 of Phifer et al. (2006), which notes that VZMS field measurements of waste content range from roughly 0.15 to 0.30 suggesting saturation levels of $35 \%$ to $75 \%$. Also, the speed of soil moisture movement in the vadose zone has been measured in the field to be approximately $v=7 \mathrm{ft} / \mathrm{yr}$ under natural recharge conditions (Horton 1975, Haskell and Hawkins 1964, Horton and Hawkins 1964). Pore velocity is computed as $v=U / n S$. Natural recharge has been estimated at about $U=15 \mathrm{in} / \mathrm{yr}$ (Hubbard and Emslie 1984) and porosity for Lower Vadose Zone sediments is $n=0.39$ (Phifer et al. 2006, Table 5-18). Saturation can then be estimated as

$$
S=\frac{U}{n v}=\frac{15 \mathrm{in} / \mathrm{yr}}{(0.39)(7 \mathrm{ft} / \mathrm{yr})} \cdot \frac{\mathrm{ft}}{12 \mathrm{in}}=46 \%
$$

which is significantly lower than $73 \%$. To assess the impact of a potential water content model bias, the Lower Vadose Zone porosity is modified from 0.39 (Phifer et al. 2006) to 0.25 in the vadose zone flow model. The modified porosity is computed as (0.39)(46\%/73\%) as a means to achieve a simulated water content similar to field measurements.

Figure 5-1 through Figure 5-6 illustrate the breakthrough curves resulting from adjusted water content. The peak concentrations are observed to be larger than those in Figure 4-3 through Figure 4-8 as expected. Using Case02 as an example, the peak concentration in the Upper_Center lysimeter increased from 411 to $516 \mathrm{pCi} / \mathrm{mL}$. Further peak concentration values are given in Table 5-1, and Table 5-2 presents the projected SOF impact resulting from adjusted water content applied to all modeling cases. The Case33 through Case36 average (see Section 4.2 for further discussion) becomes SOF $=1.05$, slightly above the performance objective.

Table 5-1. Simulated peak H-3 concentrations at UPPER and LOWER lysimeter locations w/adjusted water content.

\begin{tabular}{||l|c|c|c|c|c|c|c||}
\hline $\begin{array}{l}\text { Peak H-3 Concentration } \\
\text { from Simulated Lysimeters }\end{array}$ & Case01 & Case02 & Case03 & Case04 & Case05 & Case06 & $\begin{array}{c}\text { Case03 to } \\
\text { Case06 } \\
\text { average }\end{array}$ \\
\hline UPPER_CENTER & 111 & 516 & 348 & 278 & 376 & 136 & \\
\hline UPPER_EDGE & 107 & 419 & 294 & 228 & 498 & 425 & \\
\hline Representative UPPER & 111 & 516 & 348 & 278 & 437 & 281 & 336 \\
\hline LOWER_CENTER & 83 & 181 & 121 & 94 & 133 & 145 & \\
\hline LOWER_EDGE & 76 & 135 & 96 & 75 & 158 & 120 & \\
\hline Representative LOWER & 83 & 181 & 121 & 94 & 145 & 132 & 123 \\
\hline
\end{tabular}




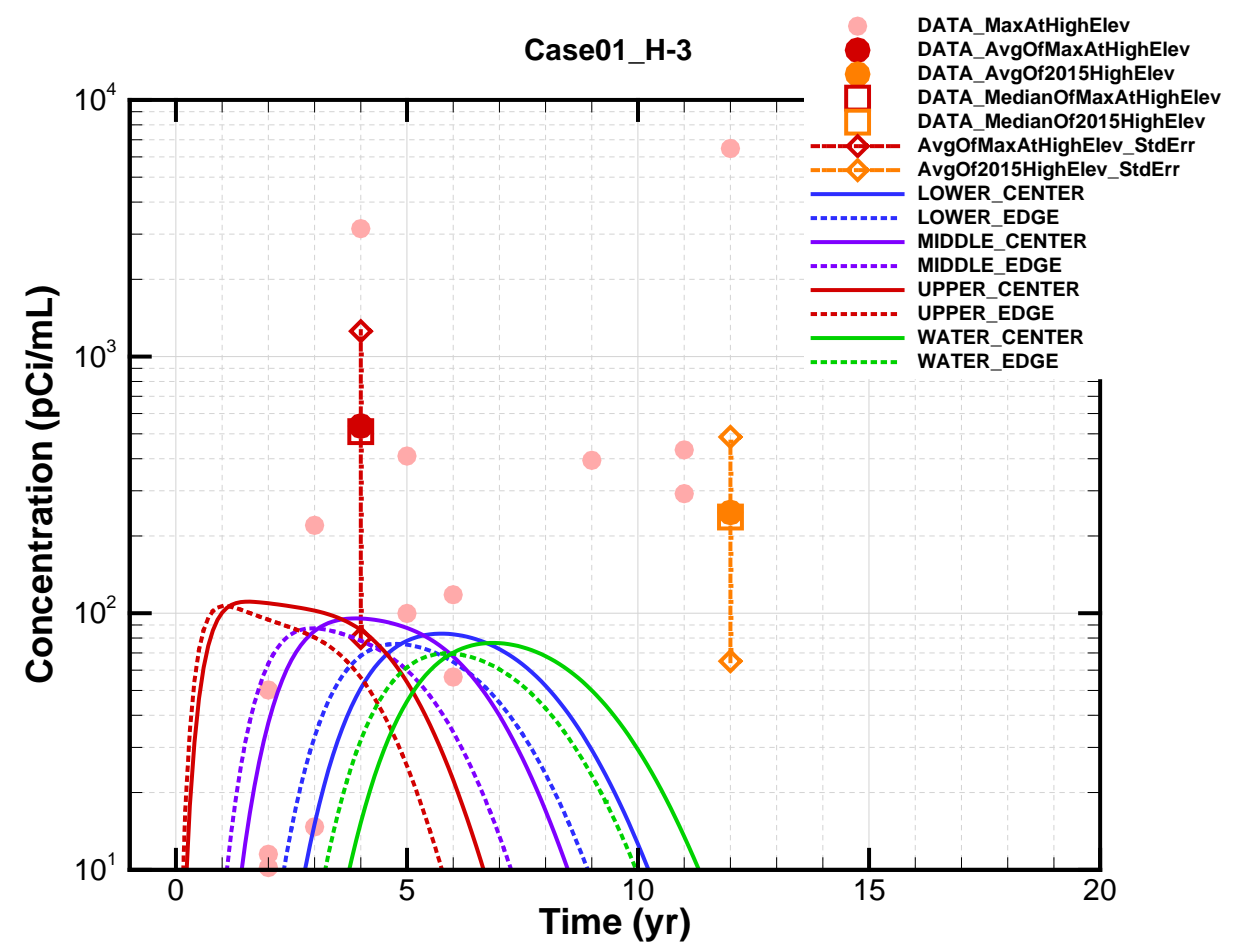

Figure 5-1. Simulated vadose zone concentrations for modeling Case01 w/adjusted water content -PA + grid refinement + no side slope usage.

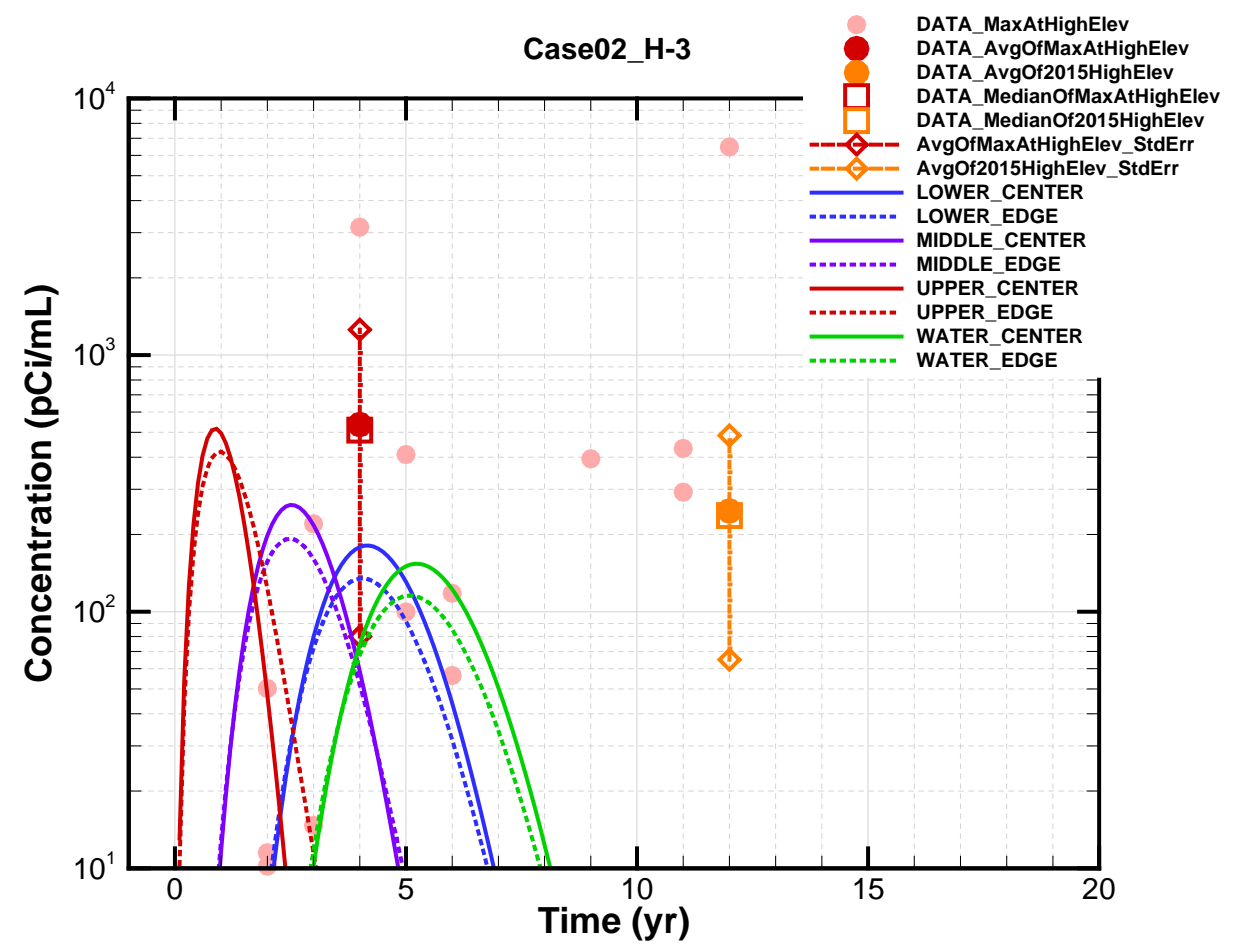

Figure 5-2. Simulated vadose zone concentrations for modeling Case02 w/adjusted water content -B-25 boxes + no dispersion. 


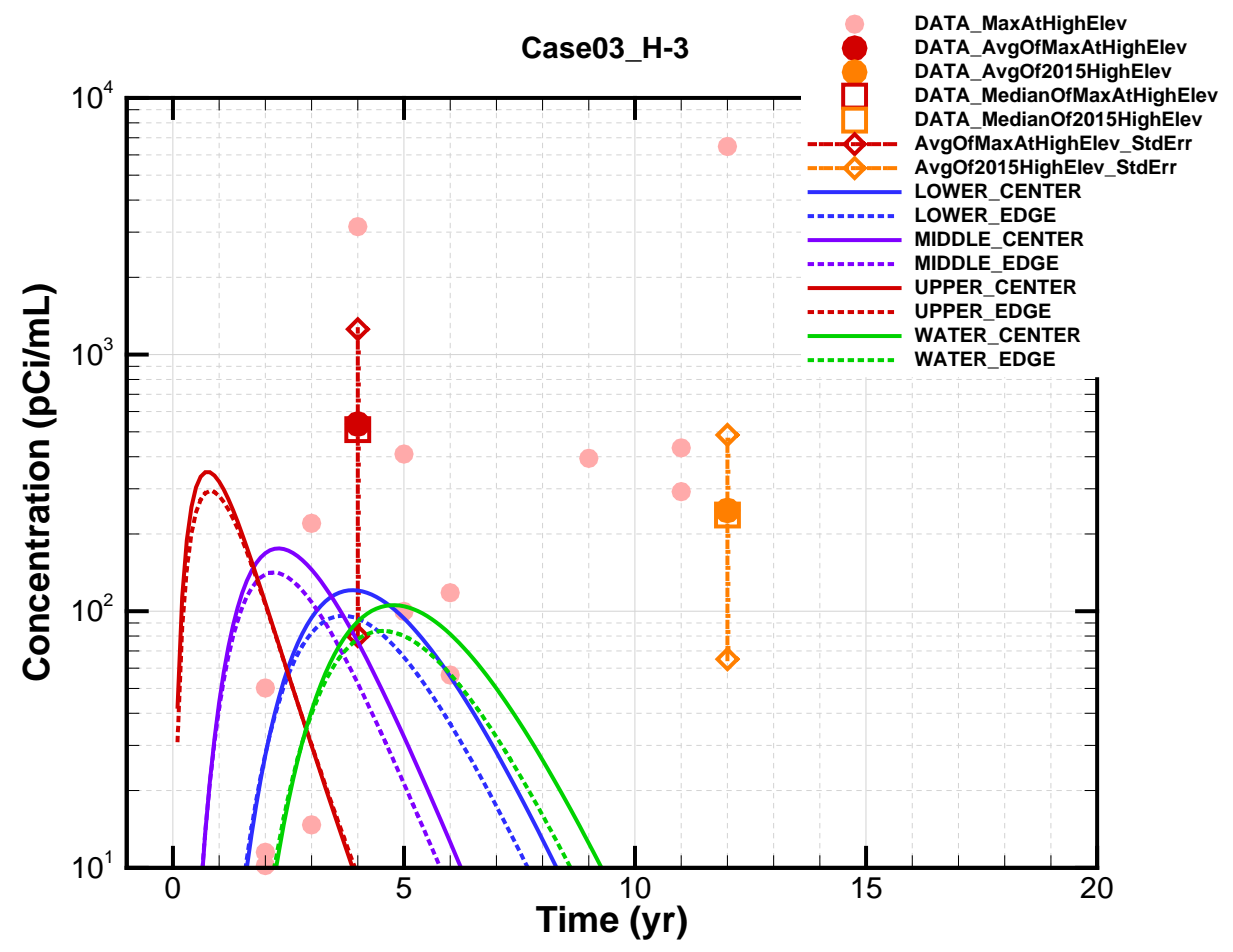

Figure 5-3. Simulated vadose zone concentrations for modeling Case03 w/adjusted water content -B-25 boxes $+5 \%$ dispersivity.

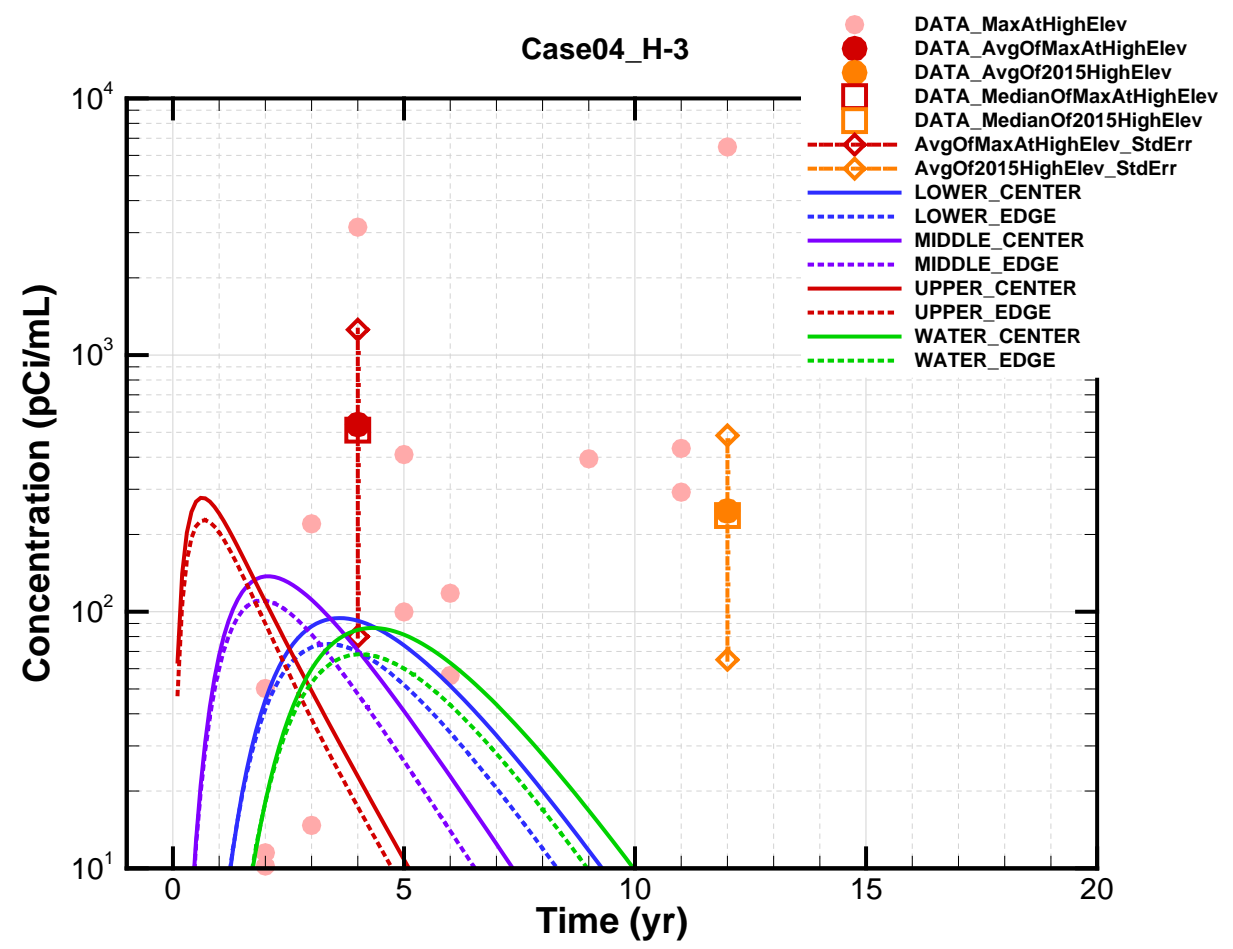

Figure 5-4. Simulated vadose zone concentrations for modeling Case04 w/adjusted water content -B-25 boxes $+10 \%$ dispersivity. 


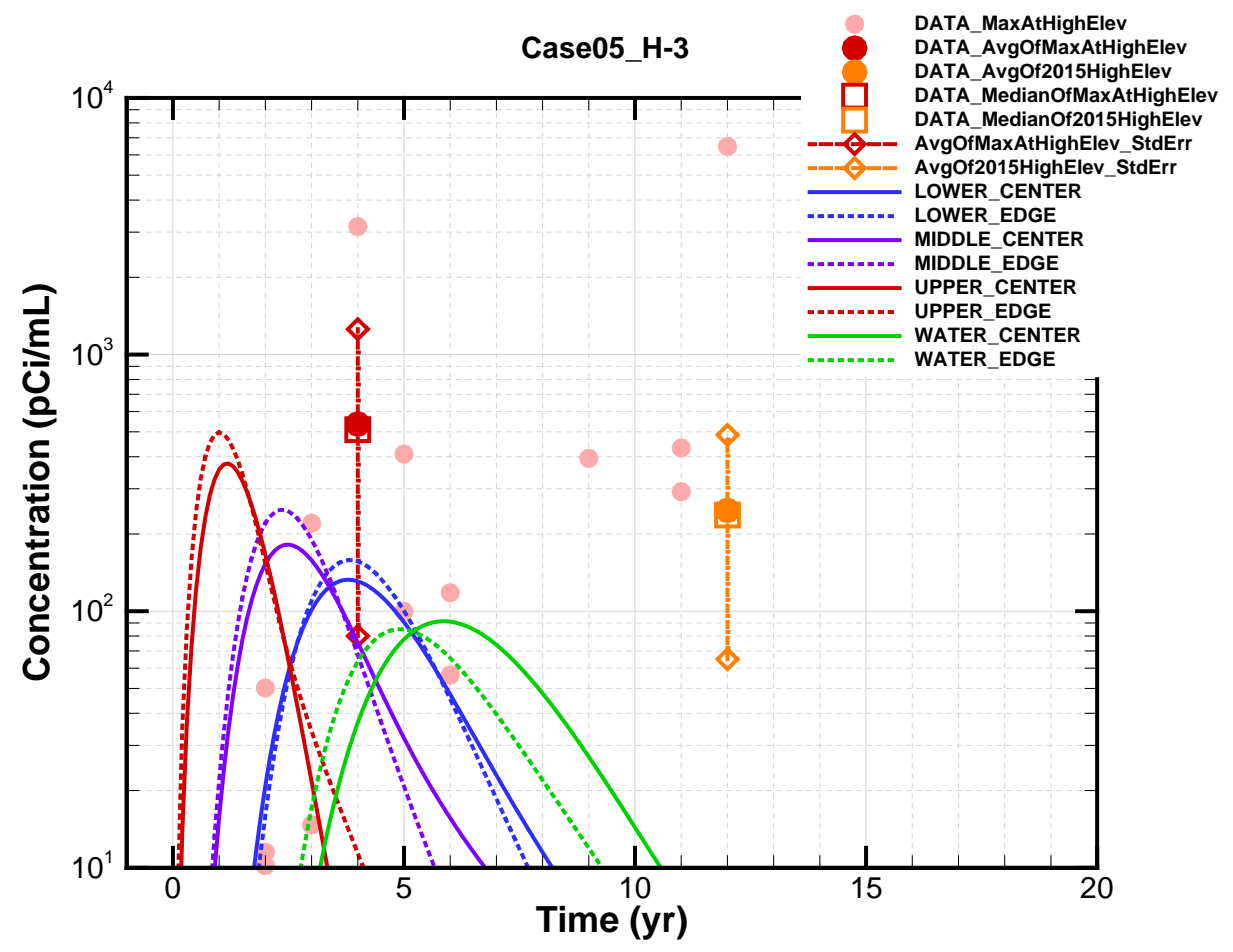

Figure 5-5. Simulated vadose zone concentrations for modeling Case05 w/adjusted water content -B25 boxes $+25 \%$ low permeability in undisturbed soil.

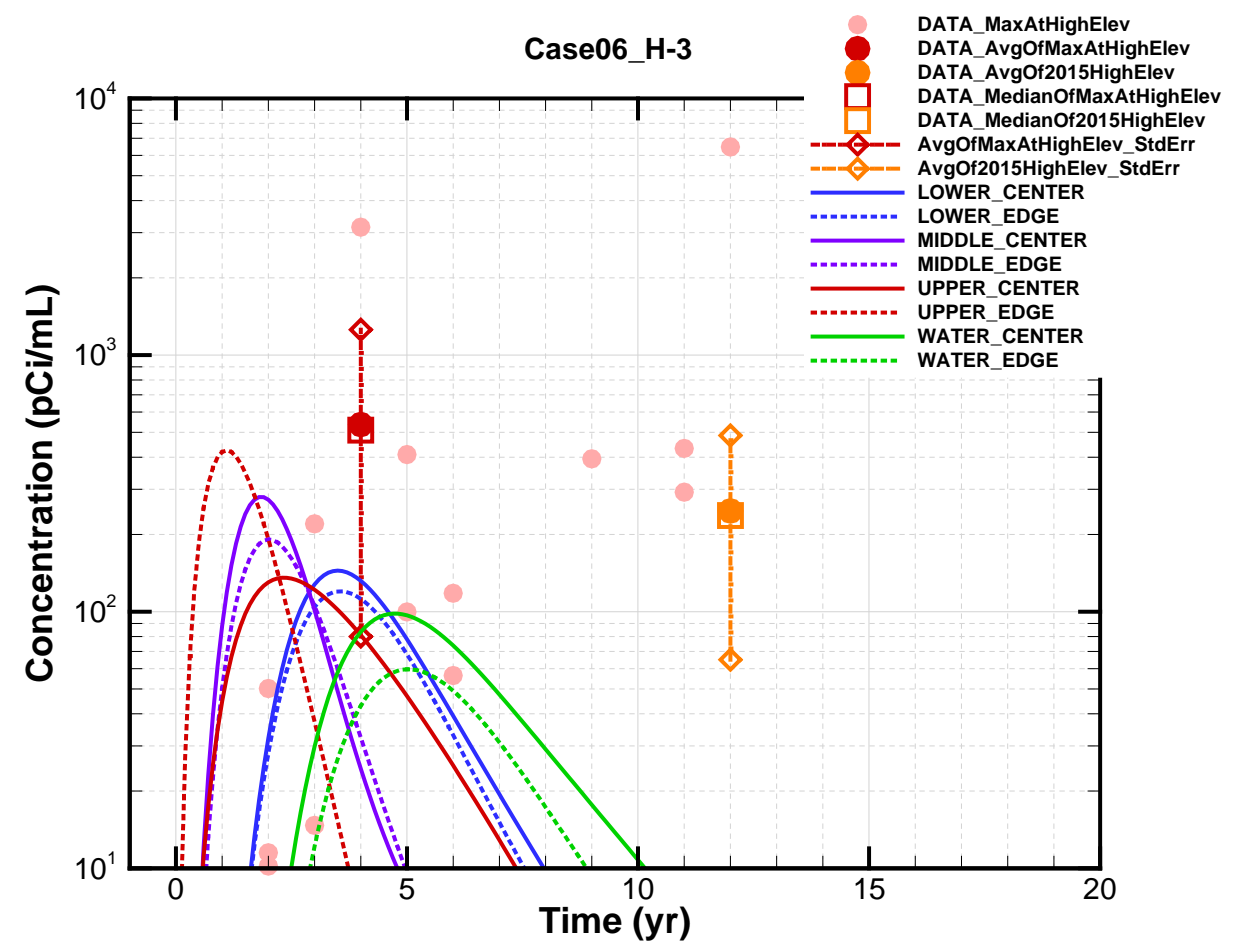

Figure 5-6. Simulated vadose zone concentrations for modeling Case06 w/adjusted water content -B25 boxes $+35 \%$ low permeability in undisturbed soil. 
Table 5-2. Projected WITS sum-of-fractions for aquifer scenarios $0 x$ through $3 x$ w/adjusted vadose zone water content.

Configuration Case00 Case01 Case02 Case03 Case04 Case05 Case06 Case10 Case11 Case12 Case13 Case14 Case15 Case16 Case20 Case21 Case22 Case23 Case24 Case25 Case26 Case30 Case31 Case32 Case33 Case34 Case35 Case36 Instantaneous disposals is

Un timing in ET1

As-disposed-of ET1 wa

Uncontained waste (soil)

Homogeneous soil: $0 \%$ low permeability

Heterogeneous soil: 25\% low permeability

Heterogeneous soil: $35 \%$ low permeability

Dispersivity $=0 \mathrm{ft}$

Dispersivity $=1.75 \mathrm{ft}(5 \%$ of $35 \mathrm{ft})$
Dispersivity $=3.5 \mathrm{ft}(10 \%$ of $35 \mathrm{ft})$
Vadosing

Vadose zone model description: PA

PA B25 box

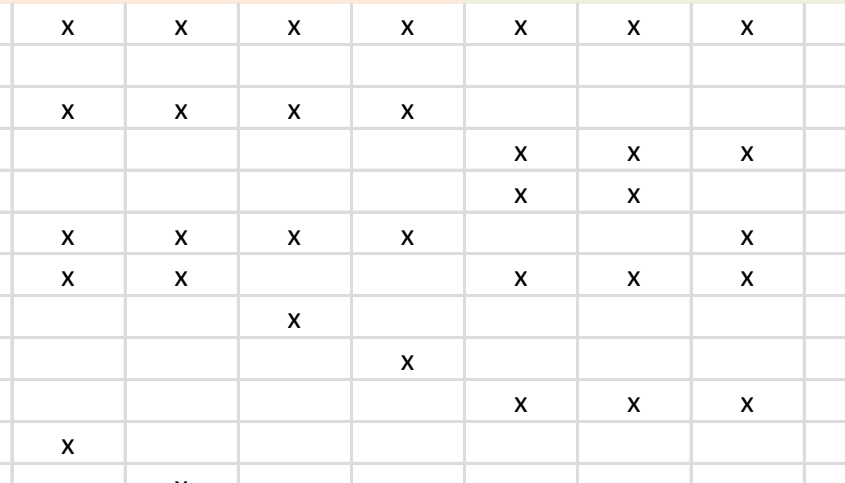

\begin{tabular}{|c|c|c|c|c|c|}
\hline PA B25 box & $B 25+$ & $B 25+$ & $B 25+$ & $B 25+$ & PA flux \\
\hline$W / n o$ & $P$
\end{tabular}

dispersi dispersi $25 \% \quad 35 \%$

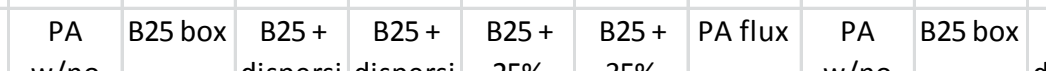

on $=$ on $=$ low low

who

dispersi dispersi $25 \% \quad 35 \%$

$5 \%$ L $10 \%$ L perm. perm. in side

10\% low low

$w /$ no

in side

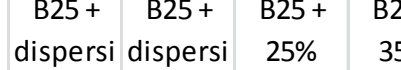

on $=$ on $=$ low low

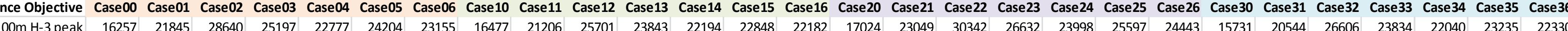

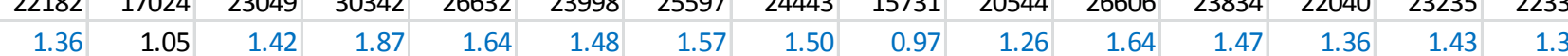

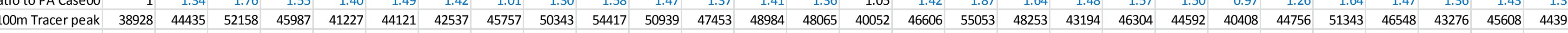
WITS inventory

WITS Projected...

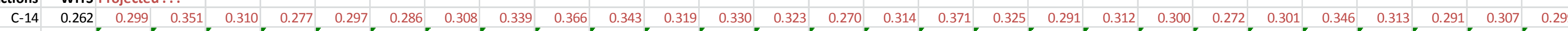

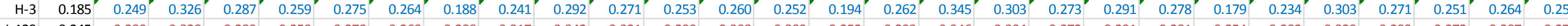

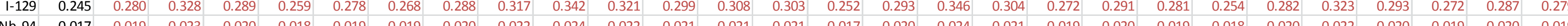

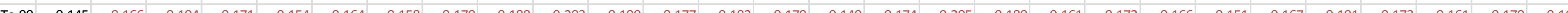

Other 0.014 SOF $\quad 0.87$

Ratio to WITS SO

1

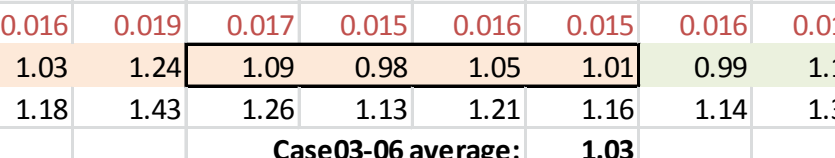

Case03-06 average: 1.03

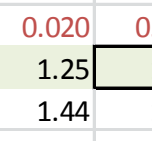

1.25 \begin{tabular}{|l|l|l|l|l|l|l|l|l|l|l}
0.018 & 0.017 & 0.018 & 0.017 & 0.014 & 0.017 & 0.020 \\
\hline 1.17 & 1.09 & 1.12 & 1.10 & 0.00 & 1.08 & 1.31 \\
\hline 1.34 & 1.25 & 1.29 & 1.26 & 1.03 & 1.24 & 1.51 \\
\hline
\end{tabular}

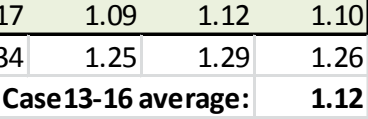

\begin{tabular}{ll}
0.90 & 1.08 \\
1.03 & 1.24 \\
\hline
\end{tabular}
Case13-16 average: $\quad \mathbf{1 . 1 2}$
Ratio to WITS SOF:

\begin{tabular}{rr}
\hline 1 \\
\hline 1 \\
1
\end{tabular}

\begin{tabular}{rrrr}
0.017 & 0.016 & 0.017 & 0.016 \\
\hline 1.15 & 1.03 & 1.10 & 1.06 \\
\hline 1.32 & 1.19 & 1.27 & 1.22
\end{tabular}

Case23-26 average: 1.09

\begin{tabular}{|lllllll}
0.015 & 0.016 & 0.018 & 0.017 & 0.016 & 0.016 & 0.016 \\
\hline 0.89 & 1.02 & 1.20 & 1.09 & 1.01 & 1.06 & 1.03
\end{tabular}

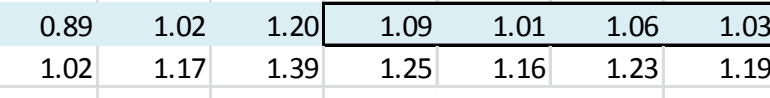

1.05 


\subsection{Discussion}

\subsection{Observed versus expected tritium concentrations in the vadose zone}

The exact nature of ET1 tritium release and migration through the vadose zone through FY2015 is inherently uncertain due to limited sampling locations and events. Similarly, model simulation outputs are inherently uncertain due to uncertain modeling assumptions and inputs. While uncertainties in data and modeling preclude making inferences with $100 \%$ confidence, certain conclusions can be drawn with reasonable confidence.

From Table 3-3, the sample average (mean) of peak tritium concentration at shallow depths is 536 $\mathrm{pCi} / \mathrm{mL}$ and the median ranges from 315 to $510 \mathrm{pCi} / \mathrm{mL}$ when the data are assumed to be drawn from a log-normal distribution, depending on the analysis method. In 2015, the mean tritium concentration at shallow depths was $247 \mathrm{pCi} / \mathrm{mL}$ and the median value using a bootstrapping method is $237 \mathrm{pCi} / \mathrm{mL}$. In comparison, representative simulated peak concentrations at shallow depths are 257 (Phifer et al. 2006 material properties) and $336 \mathrm{pCi} / \mathrm{mL}$ (adjusted water content) based on two revised models that account for predominantly B-25 box disposals (Table 4-2 and Table 5-1). These shallow lysimeter model results are similar to the data medians, and well within the $95 \%$ confidence intervals for the estimated data distributions (Table 3-3). Furthermore, the model results generally lie within the 50\% confidence intervals ( $25 \%$ to $75 \%$ cumulative probability) for shallow lysimeters. Thus little evidence exists to support a hypothesis that the simulated and actual plume concentrations are significantly different.

For the deeper vadose zone, the median of FY2015 Action Level lysimeter data is $112 \mathrm{pCi} / \mathrm{mL}$ (Table 3-3) compared to model predictions of 76 (Phifer et al. 2006 material properties) and $123 \mathrm{pCi} / \mathrm{mL}$ (adjusted water content) where waste zone properties represent B-25 box disposal. Again, the model predictions are well within the sample $95 \%$ confidence interval, and also within the $50 \%$ confidence interval, indicating consistency between the revised models and field data.

\subsection{Expectation of meeting DOE 435.1 performance objectives}

When using soil properties from Phifer et al. (2006) and assuming B-25 box disposal, Table 4-4 indicates that ET1 can be expected to meet performance objectives under as-disposed-of conditions for ET1 and ET2. In fact, a 50\% higher actual tritium inventory could be accommodated without exceeding an SOF of 1.0. When using a modified Lower Vadose Zone porosity to remove a potential bias in simulated water content, Table 5-2 indicates an SOF of $1.05,5 \%$ over the performance objective. However, the PA disposal limit calculation assumes plume interaction with disposal unit groups adjoining ET1 and ET2 (i.e. east and center Slit Trench groups), an effect that lowers ET disposal limits by 19\% (Table 6-2 of WSRC 2008). The phased operation of E-Area disposal units will minimize the potential for any inter-disposal unit group plume interaction. The assumed 19\% penalty is thus largely absent in fact, and more than compensates for the $5 \%$ overage. Therefore ET1 is not likely to exceed performance objectives.

It should be noted that the modeling results and findings presented herein are specific to the ET1 and 2 disposal unit pair and current disposal practices, and not necessarily applicable to different disposal practices and other engineered trench units, for example, ET3.

\subsection{Recommended actions}

Considering the model improvements developed herein and continued acquisition of tritium data from the VZMS, the following actions are recommended:

1) In the upcoming PA revision, the E-Area PA vadose model should be revised to account for B-25 box and similar containerized waste disposals. Furthermore, the hydraulic properties assigned to 
the vadose zone should be reassessed and revised if found to produce a significant bias in simulated moisture content.

2) Upon approval of the next PA, tritium administrative limits for action level lysimeters should be revised to reflect concentrations predicted by the revised PA vadose zone model.

3) Solid Waste should consider incorporating existing groundwater monitoring information as part of the PA monitoring program (specific actions to be developed). It is important to keep in mind that compliance with the DOE Order 435.1 performance objectives is assessed in groundwater rather than the vadose zone.

4) Vadose zone monitoring should continue to be maintained and expanded for trench units because it provides an early indication of trench disposal unit performance relative to PA assumptions and modeling forecasts, and addresses GW monitoring limitations due to the existing Mixed Waste Management Facility tritium plume beneath E-Area. 
SRNL-STI-2016-00546

Revision 0

\subsection{References}

Box, G. E. P., W. G. Hunter and J. S. Hunter. Statistics for Experimenters. John Wiley \& Sons, New York. 653 p. 1978.

Department of Energy. Radioactive Waste Management. DOE Order 435.1. Certified: 1-9-07.

Efron, B. The jackknife, the bootstrap and other resampling plans. CBMS-NSF Regional Conference Series in Applied Mathematics. Number 38 Society for Industrial and Applied Mathematics, Philadelphia, Pennsylvania. 1982.

Flach, G. P. An Evaluation of Dual-Media Contaminant Transport for SRS Environmental Applications (U). WSRC-TR-2002-00291, Revision 1. October 2002.

Flach, G. P. Groundwater Flow Model of the General Separations Area Using PORFLOW (U). WSRCTR-2004-00106, Revision 0. July 2004.

Haskell, C. C. and R. H. Hawkins. D2O - Na24 method for tracing soil moisture movement in the field. Soil Science Society of America Proceedings, v28, 725-728. 1964.

Hiergesell, R. A., M. R. Millings, G. K. Humphries and D. F. Sink. FY2014 Performance Assessment Annual Review for the E-Area Low-Level Waste Facility. SRNL-STI-2014-00582, Revision 0. January 2015.

Hiergesell, R. A., M. R. Millings, G. K. Humphries and D. F. Sink. FY2015 Performance Assessment Annual Review for the E-Area Low-Level Waste Facility. SRNL-STI-2015-00691, Revision 0. January 2016.

Horton, R. H. Soil moisture flow as related to the burial of solid radioactive waste. DPST-75-218. 1975.

Horton, J. H. and R. H. Hawkins. Flow path of rain from the soil surface to the water table. Soil Science, v100, 377-383. 1964.

Hubbard, J. E. and R. H. Emslie. Water Budget for SRP Burial Ground Area. DPST-83-742. 1984.

Millings, M. R. Performance Assessment Monitoring Plan for the E-Area Low Level Waste Facility. SRNL-RP-2009-00534, Revision 0. April 2009.

Millings, M. R. Performance Assessment Monitoring Plan for the E-Area Low Level Waste Facility. SRNL-RP-2009-00534, Revision 1. August 2012.

Nuclear Regulatory Commission. Appendix I to Part 73-Category 1 and 2 Radioactive Materials. http://www.nrc.gov/reading-rm/doc-collections/cfr/part073/part073-appi.html. Accessed 29 September 2016.

Phifer, M. A., M. R. Millings and G. P. Flach. Hydraulic Property Data Package for the E-Area and ZArea Soils, Cementitious Materials, and Waste Zones. WSRC-STI-2006-00198, Revision 0. September 2006. 
Phifer, M. A. and E. L. Wilhite. Waste Subsidence Potential Versus Supercompaction. WSRC-RP-200100613. September 2001.

Swingle, R. F. Special Analysis: Revised Groundwater Protection and All-Pathways Limits for E-Area Low-Level Waste Facility Trenches. SRNL-STI-2012-00466, Revision 0. August 2012.

Swingle, R. F. Revision of the ELLWF Disposal Limits Database Adding Revised Trench Limits (Revision 2012-2). Memorandum SRNL-L3200-2012-00038. December 4, 2012.

Washington Savannah River Company LLC. E-Area Low-Level Waste Facility DOE 435.1 Performance Assessment. WSRC-STI-2007-00306, Rev. 0. July 2008.

Zheng, C. and G. D. Bennett. Applied Contaminant Transport Modeling: Theory and Practice. van Nostrand Reinhold, New York. 440 p. 1995. 


\section{Appendix A. Effective Properties of a Blended Material}

\section{Selected symbols}

- $V=$ volume

- $m=$ mass

- $F=$ flow / flux

\section{Subscripts}

- $\quad$ none $=$ total or effective

- $i=$ component

- $v=$ void volume

- $w=$ water volume

- $s=$ solid phase

Volume fraction

$$
\begin{gathered}
V=V_{1}+V_{2} \\
1=\frac{V_{1}}{V}+\frac{V_{2}}{V}=f_{1}+f_{2}
\end{gathered}
$$

\section{Porosity}

$$
\begin{gathered}
V_{v}=V_{v 1}+V_{v 2} \\
n=\frac{V_{v}}{V}=\frac{V_{v 1}}{V_{1}} \frac{V_{1}}{V}+\cdots=f_{1} n_{1}+f_{2} n_{2}
\end{gathered}
$$

\section{Bulk density}

$$
\begin{gathered}
m_{s}=m_{s 1}+m_{s 2} \\
\rho=\frac{m_{s}}{V}=\frac{m_{s 1}}{V_{1}} \frac{V_{1}}{V}+\cdots=f_{1} \rho_{1}+f_{2} \rho_{2}
\end{gathered}
$$

Solid density

$$
\begin{gathered}
\rho_{s}=\frac{m_{s}}{V_{s}}=\frac{m_{s 1}}{V_{s 1}} \frac{V_{s 1}}{V} \frac{V}{V_{s}}+\cdots=\rho_{s 1}\left(\frac{V_{1}-V_{v 1}}{V} \cdot \frac{V}{V-V_{v}}\right)+\cdots \\
=\rho_{s 1}\left[\left(1-V_{v 1} / V_{1}\right) \frac{V_{1}}{V} \cdot \frac{V}{V-V_{v}}\right]+\cdots=\rho_{s 1}\left[\left(1-V_{v 1} / V_{1}\right) \frac{V_{1}}{V} \cdot \frac{1}{1-V_{v} / V}\right]+\cdots
\end{gathered}
$$




$$
=\rho_{s 1}\left[\left(1-n_{1}\right) f_{1} \cdot \frac{1}{1-n}\right]+\cdots=\frac{f_{1}\left(1-n_{1}\right) \rho_{s 1}+f_{2}\left(1-n_{2}\right) \rho_{s 2}}{1-n}
$$

\section{Water content}

$$
\begin{gathered}
V_{w}=V_{w 1}+V_{w 2} \\
\theta=\frac{V_{w}}{V}=\frac{V_{w 1}}{V_{1}} \frac{V_{1}}{V}+\cdots=f_{1} \theta_{1}+f_{2} \theta_{2}
\end{gathered}
$$

Saturation

$$
\begin{gathered}
\theta=S n=f_{1} \theta_{1}+\cdots=f_{1} S_{1} n_{1}+\cdots \\
S=\frac{f_{1} S_{1} n_{1}+f_{2} S_{2} n_{2}}{n}
\end{gathered}
$$

Saturated hydraulic conductivity (and unsaturated conductivity)

$$
\begin{gathered}
F=F_{1}+F_{2} \\
-A K \frac{d h}{d z}=-A_{1} K_{1} \frac{d h}{d z}+\cdots \\
K=\frac{A_{1}}{A} K_{1}+\cdots=\frac{V_{1}}{V} K_{1}+\cdots=f_{1} K_{1}+f_{2} K_{2} \\
K=f_{1} K_{1}+f_{2} K_{2}
\end{gathered}
$$

\section{Relative permeability}

$$
\begin{gathered}
k_{r} K=f_{1} k_{r 1} K_{1}+\cdots \\
k_{r}=\frac{f_{1} k_{r 1} K_{1}+f_{2} k_{r 2} K_{2}}{K}
\end{gathered}
$$




\section{Summary}

$$
\begin{gathered}
1=f_{1}+f_{2} \\
n=f_{1} n_{1}+f_{2} n_{2} \\
\rho=f_{1} \rho_{1}+f_{2} \rho_{2} \\
\rho_{s}=\frac{f_{1}\left(1-n_{1}\right) \rho_{s 1}+f_{2}\left(1-n_{2}\right) \rho_{s 2}}{1-n} \\
\theta=f_{1} \theta_{1}+f_{2} \theta_{2} \\
S=\frac{f_{1} S_{1} n_{1}+f_{2} S_{2} n_{2}}{n} \\
K=\frac{f_{1} K_{1}+f_{2} K_{2}}{K} \\
k_{r}=\frac{f_{1} k_{r 1} K_{1}+f_{2} k_{r 2} K_{2}}{K}
\end{gathered}
$$




\section{Special case}

$$
\begin{gathered}
n_{2}=1 \\
S_{2}=0 \\
K_{2}=0 \\
n=f_{1} n_{1}+f_{2} \\
\rho=f_{1} \rho_{1} \\
\rho_{s}=\rho_{s 1} \\
\theta=f_{1} \theta_{1} \\
S=\frac{f_{1} S_{1} n_{1}}{n} \\
K=f_{1} K_{1} \\
k_{r}=k_{r 1}
\end{gathered}
$$


SRNL-STI-2016-00546

Revision 0

This page intentionally left blank 


\section{Distribution:}

T. B. Brown, 773-A

D. A. Crowley, 773-42A

D. E. Dooley, 773-A

P. N. Fairchild, 704-55E

A. P. Fellinger. 773-42A

S. D. Fink, 773-A

J. C. Griffin, 773-A

C. C. Herman, 773-A

D. T. Hobbs, 773-A

E. N. Hoffman, 999-W

J. E. Hyatt, 773-A

K. M. Kostelnik, 773-42A

B. B. Looney, 773-42A

T. O. Oliver, 773-42A

F. M. Pennebaker, 773-42A

B. J. Wiedenman, 773-42A

W. R. Wilmarth, 773-A

EM File, 773-42A, Rm 243

(1 file copy and 1 electronic copy)

Records Administration (EDWS)
S. E. Aleman, 735-A

B. T. Butcher, 773-42A

T. L. Danielson, 703-41A

J. A. Dyer, 703-41A

P. N. Fairchild, 704-55E

G. P. Flach, 773-42A

F. L. Fox Jr., 704-59E

N. V. Halverson, 773-42A

L. L. Hamm, 735-A

T. Hang, 773-42A

G. K. Humphries, 704-59E

D. I. Kaplan, 773-42A

D. Li, 773-42A

M. G. Looper, 704-36E

R. R. Seitz, 773-42A

D. F. Sink, 704-56E

F. G. Smith, III 773-42A

I. J. Stewart, 704-58E

T. S. Whiteside, 773-42A

J. L. Wohlwend, 703-41A 